\begin{tabular}{lc|c|c|}
\hline Bentham OPEN & The Open Ophthalmology Journal & $\begin{array}{l}\text { The Open } \\
\text { Ophthalmology } \\
\text { lournal }\end{array}$ \\
\hline CrossMark & Content list available at: www.benthamopen.com/TOOPHTJ & DOI: $10.2174 / 1874364101812010181$ \\
\hline
\end{tabular}

REVIEW ARTICLE

\title{
A Review of Corneal Collagen Cross-linking - Current Trends in Practice Applications
}

\author{
Li Lim ${ }^{1, *}$ and Elizabeth Wen Ling Lim ${ }^{2}$ \\ ${ }^{I} M B B S$ (Singapore), MMed (Ophth), FRCS(Ed), FAMS (S'pore) Senior Consultant, Corneal and External Eye Disease \\ Service, Singapore National Eye Centre, Singapore, Singapore \\ ${ }^{2}$ Undergraduate medical student, Yong Loo Lin School of Medicine, National University of Singapore, Singapore, \\ Singapore
}

Received: July 07, 2017

Revised: December 05, 2017

Accepted: January 22, 2018

\section{Abstract:}

Objective:

To review the literature on current applications of corneal Collagen Cross-Linking (CXL).

\section{Methods:}

A review of publications on corneal cross-linking was conducted. This included systemic reviews, randomized controlled clinical trials, cohort studies, case-controlled studies and case series. A summary of the publications is tabulated.

\section{Results:}

The original indication of riboflavin - Ultraviolet-A (UVA) induced corneal collagen cross-linking is to arrest the progression of keratoconus. Studies show that it is effective in arresting the progression of keratoconus and post-LASIK ectasia with the standard Dresden protocol (epithelium-off). There are also improvements in visual, keratometric and topographic measurements over time. Severe complications of cross-linking are rare. The epithelium-on techniques have less efficacy than the Dresden protocol. Accelerated protocols have variable results, with some studies reporting comparable outcomes to the Dresden protocol while other studies reporting less efficacious outcomes. Cross-linking combined with refractive procedures provide better visual outcome but long term studies are warranted. Cross-linking for the treatment of infective keratitis is a promising new treatment modality. Initial studies show that it is more effective for superficial rather than deep infections and for bacterial rather than fungal infections.

\section{Conclusions:}

Corneal cross-linking is a procedure with an expanding list of indications from the treatment of corneal ectasias to infective keratitis. While the standard Dresden protocol is established as the gold standard treatment for progressive keratoconus, the more recent protocols may require further refinements, investigative and long-term studies.

Keywords: Cornea collagen cross-linking, post-LASIK ectasia, Keratoconus, Infective keratitis, UVA, Dresden protocol.

\section{INTRODUCTION}

Wollensak, Spoerl and Seiler reported the first clinical study on riboflavin - UVA induced corneal collagen crosslinking procedure for the treatment of progressive keratoconus in adults [1]. Since then, corneal cross-linking has been widely used for the treatment of progressive keratoconus as well as other conditions including post-LASIK ectasia. Corneal cross-linking is also used in conjunction with laser vision correction procedures and intrastromal ring procedures. More recently, it is used to treat infective keratitis. The purpose of this paper is to provide a review of the

\footnotetext{
* Address correspondence to this author at the Singapore National Eye Centre, 11 Third Hospital Avenue, Singapore 168751, Singapore;
} Tel: +6562277255; E-mail: lim.li@singhealth.com.sg 
current trends in practice applications of corneal collagen cross-linking.

\section{OVERVIEW}

Epithelium-off CXL (Dresden protocol)

Epithelium-on CXL

Accelerated CXL

CXL plus (Cross-linking combined with Refractive Procedures for the treatment of Corneal Ectatic Disorders)

Customised CXL

Combined laser in-situ keratomileusis(LASIK) and accelerated CXL

Cross-linking for the treatment of Infective Keratitis (PACK-CXL)

Other Applications of CXL

Cross-linking complications

\section{STANDARD EPITHELIUM-OFF CXL PROTOCOL (DRESDEN PROTOCOL)}

Wollensak, Spoerl and Seiler introduced the Dresden protocol that has become the standard procedure of crosslinking today [1]. A documented evidence of progression of the keratoconus should be obtained before performing cross-linking. Studies show that progression may be defined as a more than 1 Diopter (D) increase in Kmax (maximum keratometry) and/or a more than 1D increase in average keratometry and/or refractive astigmatism of more than 1D and/or decrease in pachymetry of more than $10 \%$ in the preceding $12-18$ months [2 - 4]. A minimum corneal stromal thickness of 400um is recommended prior to surgery for the safety of the corneal endothelium [5].

The cross-linking procedure is performed under topical anesthesia (eg tetracaine 1\%). An epithelial debridement $(8-9 \mathrm{~mm})$ is performed followed by instillation of riboflavin $0.1 \%$ eyedrops for 30 minutes at 2 minute intervals. The riboflavin solution may contain $20 \%$ dextran as in the earlier studies [1] or may be dextran free containing hydroxypropyl methylcellulose [6]. The dextran-free riboflavin preparation may reduce the incidence of stromal dehydration during the procedure. At the end of the riboflavin instillation, ultrasound pachymetry is performed at the centre of the cornea and if the corneal thickness is below 400um, hypotonic riboflavin eyedrops are applied until the corneal thickness returns to a minimum of 400 um thickness. The cornea is then irradiated with the Ultraviolet A (UVA) device at $3 \mathrm{~mW} / \mathrm{cm}^{2}$ for 30 minutes with continued instillation of riboflavin eyedrops at 2-minute intervals. After irradiation is completed, topical antibiotics/steroids and bandage lens are applied.

A corneal stromal demarcation line can sometimes be detected at the slit lamp or with the Anterior Segment Optical Coherence Tomography (AS-OCT) and is most apparent in the first 3 months after cross-linking [7]. Keratocyte apoptosis is observed anterior to the demarcation line on confocal microscopy and repopulation of keratocytes occurs after 3 months. The depth of the demarcation line has commonly been interpreted as the depth of cross-linking [8,9]. 


\section{STABILISING ADULT KERATOCONUS AND POST-LASIK ECTASIA (TABLES 1A, 1B, 1C and 2)}

Table 1A. Summary of outcomes for standard epithelium-off cross-linking. (Prospective randomised studies) $\left(3 \mathrm{~mW} / \mathrm{cm}^{2} \mathrm{UV}-\mathrm{A} \mathrm{exposure}, 5.4 \mathrm{~J} / \mathrm{cm}^{2}\right)$.

\begin{tabular}{|c|c|c|c|c|c|c|c|c|c|c|c|}
\hline Study & Study design/Indication & $\begin{array}{l}\text { No. of } \\
\text { Eves }\end{array}$ & $\begin{array}{c}\text { Follow-up, } \\
\text { Months }\end{array}$ & $\begin{array}{l}\text { Criteria for } \\
\text { Progression }\end{array}$ & $\begin{array}{l}\text { UV device/ } \\
\text { Riboflavin }\end{array}$ & \multicolumn{6}{|c|}{ Outcome } \\
\hline- & - & - & - & - & - & Overall & Pre-op K (D) & $\Delta K(D)$ & $\triangle \mathrm{UCVA}$ & $\triangle \mathrm{BCVA}$ & $\begin{array}{l}\Delta \text { Refraction } \\
\text { (D) }\end{array}$ \\
\hline $\begin{array}{l}\text { Henriquez et } \\
a l, 2011[14]\end{array}$ & $\begin{array}{c}\text { Prospective, randomised/ } \\
\text { Keratoconus }\end{array}$ & $\begin{array}{c}20 ; 10 \\
\text { treated, } 10 \\
\text { FE control }\end{array}$ & 12 & $\begin{array}{c}\text { 1. } \uparrow \text { Kmax of } 1.00 \mathrm{D} / 1 \\
\text { year } \\
\text { 2. } \downarrow \text { visual acuity } \\
\text { 3. New contact lens } \\
\text { fitting }>\text { once in } 2 \text { years }\end{array}$ & $\begin{array}{c}\text { UV-X 1000; IROC } \\
\text { AG/ Riboflavin } \\
0.1 \% \text { w dextran }\end{array}$ & $\begin{array}{c}\text { Improved UCVA. } \\
\text { Reduction in mean-K, } \\
\text { max-K and min-K, mean } \\
\text { SE, anterior and } \\
\text { posterior elevation } \\
\text { values. }\end{array}$ & - & \begin{tabular}{|c} 
Mean Max K \\
(treated): \\
$-2.66(\mathrm{P}=$ \\
$0.04)$
\end{tabular} & $\begin{array}{c}\text { Treated: From } \\
1.18 \pm 0.80 \text { to } \\
0.46 \pm 0.36 \\
\text { (LogMAR) (P } \\
<0.001)\end{array}$ & $\begin{array}{l}\text { Treated: From } \\
0.20 \pm 0.18 \text { to } \\
0.09 \pm 0.09 \\
\text { (LogMAR) (P } \\
\quad=0.06)\end{array}$ & $\begin{array}{c}\text { Mean SE } \\
\text { (treated): }-2.25 \\
(\mathrm{P}=0.01)\end{array}$ \\
\hline $\begin{array}{l}\text { Hersh et al, } \\
2011[28]\end{array}$ & $\begin{array}{c}\text { Prospective randomised/ } \\
\text { Keratoconus and post-laser } \\
\text { ectasia }\end{array}$ & \begin{tabular}{|c|}
$142 ; 71$ \\
treated, 41 \\
sham \\
control, 30 \\
FE control
\end{tabular} & 12 & $\begin{array}{c}\uparrow 1 \mathrm{D} \text { in steepest } \mathrm{K}, 1 \mathrm{D} \\
\text { cyl, } 0.5 \mathrm{D} \text { MRSE } / 24 \\
\text { months }\end{array}$ & $\begin{array}{l}\text { UV-X; IROC AG/ } \\
\text { Riboflavin } 0.1 \% \mathrm{w} \\
\text { dextran } 20 \%\end{array}$ & $\begin{array}{l}\text { Improved UDVA and } \\
\text { CDVA. Reduced max-K } \\
\text { and mean-K. }\end{array}$ & $\left.\begin{array}{c}\text { Mean } \max K \\
\text { (treated): } 58.6 \\
\pm 9.62\end{array}\right]$ & \begin{tabular}{|c|} 
Treated: $-1.7 \pm$ \\
$3.9(\mathrm{P}<$ \\
$0.001)$
\end{tabular} & $\begin{array}{c}\text { Treated: From } \\
0.84 \pm 0.34 \text { to } \\
0.77 \pm 0.37 \\
\text { (LogMAR) (P } \\
=0.04)\end{array}$ & \begin{tabular}{|c|} 
Treated: From \\
$0.35 \pm 0.24$ to \\
$0.23 \pm 0.21$ \\
(LogMAR) (P \\
$<0.001)$
\end{tabular} & $\begin{array}{c}\text { MRSE } \\
\text { (treated): }-0.86 \\
(\mathrm{P}=0.07)\end{array}$ \\
\hline $\begin{array}{l}\text { O'Brart et al, } \\
2011[2]\end{array}$ & $\begin{array}{l}\text { Blind, randomised, } \\
\text { prospective, } \\
\text { bilateral/Keratoconus }\end{array}$ & $\begin{array}{c}46 ; 24 \\
\text { treated, } 22 \\
\text { FE control }\end{array}$ & 18 & $\begin{array}{c}\text { 1. } \downarrow \text { UCVA/ BSCVA }>1 \\
\text { line } \\
\text { 2. Worsening } \\
\text { refractive/corneal } \\
\text { astigmatism, K or cone } \\
\text { apex power by } 0.75 \mathrm{D} / 18 \\
\text { months }\end{array}$ & $\begin{array}{c}\text { In-house } \\
\text { manufactured device } \\
\text { using Roithner } \\
\text { Lasertechnik diodes } \\
\text { and CMB Vega X- } \\
\text { linker/ Riboflavin } \\
0.1 \%\end{array}$ & \begin{tabular}{c|} 
Improved BSCVA. \\
Reduced Orbscan II- \\
simulated K, 3mm K, \\
simulated astigmatism, \\
cone apex power, root \\
mean square, coma, \\
spherical aberration, \\
secondary astigmatism \\
and pentafoil \\
\end{tabular} & $\begin{array}{c}\text { Mean SIM K } \\
\text { (treated): } 47.1 \\
\text { Mean SIM K } \\
\text { (control): } 47.8\end{array}$ & $\begin{array}{c}\text { Treated: }-0.62 \\
(\mathrm{P}<0.001) \\
\text { Control: }+0.14 \\
(\mathrm{P}=0.3)\end{array}$ & $\begin{array}{c}\text { Treated: }+0.06 \\
\text { (SDE) } \\
\text { Control: }-0.01 \\
\text { (SDE) } \\
(\mathrm{P}=0.2)\end{array}$ & $\begin{array}{c}\text { Treated: }+0.12 \\
\text { (SDE) } \\
\text { Control: }+0.13 \\
(\mathrm{SDE}) \\
(\mathrm{P}=0.01)\end{array}$ & $\begin{array}{l}\text { Mean SE } \\
\text { (treated): } \\
\quad+0.82 \\
\text { Mean SE } \\
\text { (control): } \\
+0.11 \\
(\mathrm{P}=0.2)\end{array}$ \\
\hline $\begin{array}{c}\text { Wittig-Silva et } \\
a l, 2008[3]\end{array}$ & $\begin{array}{l}\text { Prospective, randomised/ } \\
\text { Keratoconus }\end{array}$ & $\begin{array}{c}66: 33 \\
\text { treated; } 33 \\
\text { control }\end{array}$ & 12 & \begin{tabular}{|c|}
$1 . \uparrow \geq 1 \mathrm{D}$ in Kmax \\
$2 . \uparrow$ astigmatism with \\
manifest subjective \\
refraction $\geq 1 \mathrm{D}$ \\
3. $\uparrow$ of $0.50 \mathrm{D}$ in MRSE \\
$4 . \downarrow \geq 0.1 \mathrm{~mm}$ in back \\
optic zone radius of best \\
fitting contact lens \\
\end{tabular} & $\begin{array}{l}\text { IROC UV-X/ } \\
\text { Riboflavin } 0.1 \% \mathrm{w} \\
\text { dextran } 20 \%\end{array}$ & $\begin{array}{l}\text { Improved BSCVA and } \\
\text { reduced Kmax. }\end{array}$ & $\begin{array}{c}\text { Mean Kmax } \\
\text { (treated): } 52.70 \\
\pm 4.5 \\
\text { Mean Kmax } \\
\text { (control): } \\
50.80 \pm 4.30 \\
(\mathrm{P}=0.073)\end{array}$ & $\begin{array}{c}\text { Treated: }-1.45 \\
\pm 1.00(\mathrm{P}< \\
0.002) \\
\text { Control: }+1.28 \\
(\mathrm{P}<0.001)\end{array}$ & - & $\begin{array}{c}\text { Treated: }-0.12 \\
(\mathrm{P}=0.07) \\
\text { (LogMAR) }\end{array}$ & $\begin{array}{c}\text { MRSE: no diff } \\
\text { in both treated } \\
\text { and control }\end{array}$ \\
\hline
\end{tabular}


(Table 1\$)7223+7- - [00000000 contd....

\begin{tabular}{|c|c|c|c|c|c|c|c|c|c|c|c|}
\hline Study & Study design/ Indication & No. of & Follow-up, & Criteria for & UV device/ & \multicolumn{6}{|c|}{ Outcome } \\
\hline - & - & - & - & - & - & Overall & Pre-op K (D) & $\Delta K(D)$ & $\triangle \mathrm{UCVA}$ & $\triangle B C V A$ & \begin{tabular}{|c|}
$\Delta$ Refraction \\
(D)
\end{tabular} \\
\hline $\begin{array}{l}\text { Wittig-Silva et } \\
a l, 2014[4]\end{array}$ & $\begin{array}{l}\text { Prospective, randomised/ } \\
\text { Keratoconus }\end{array}$ & $\begin{array}{l}46 \text { treated, } \\
48 \text { control }\end{array}$ & 36 & \begin{tabular}{|c|} 
Subjective $\downarrow$ in vision \\
and $\geq 1$ of the following \\
in 12 months: \\
$1 . \uparrow \geq 1 \mathrm{D}$ in steepest \\
simulated $\mathrm{K}$ \\
$2 . \uparrow$ astigmatism with \\
manifest subjective \\
refraction $\geq 1 \mathrm{D}$ \\
3. $\downarrow \geq 0.1 \mathrm{~mm}$ in back \\
optic zone radius of best \\
fitting contact lens
\end{tabular} & $\begin{array}{l}\text { UV-X 1000; IROC/ } \\
\text { Riboflavin } 0.1 \% \mathrm{w} \\
\text { dextran } 20 \%\end{array}$ & $\begin{array}{l}\text { Improved UCVA and } \\
\text { BSCVA, reduction in } \\
\text { Kmax. Significant } \\
\text { reduction in corneal } \\
\text { thickness. }\end{array}$ & $\begin{array}{c}\text { Kmax } \\
\text { (treated): } 52.87 \\
\pm 4.31 \\
\text { Kmax } \\
\text { (control): } \\
51.18 \pm 4.03 \\
(\mathrm{P}=0.052)\end{array}$ & \begin{tabular}{|c|} 
Treated: -1.03 \\
\pm 0.19 \\
Control: +1.75 \\
\pm 0.38 \\
$(\mathrm{P}<0.001)$
\end{tabular} & $\begin{array}{c}\text { Treated: }-0.15 \\
\pm 0.06 \\
\text { Control: }+0.10 \\
\pm 0.04 \\
\text { (LogMAR) } \\
(\mathrm{P}=0.001)\end{array}$ & $\begin{array}{l}\text { Treated: }-0.09 \\
\quad \pm 0.03 \\
\text { Control: }-0.05 \\
\quad \pm 0.03 \\
\text { (LogMAR) (P } \\
\quad=0.347 \text { ) }\end{array}$ & $\begin{array}{l}\text { Treated: }-0.61 \\
\quad \pm 0.41 \\
\text { Control: }-0.79 \\
\pm 0.42 \\
(\mathrm{P}=0.752)\end{array}$ \\
\hline $\begin{array}{l}\text { Lang et al, } \\
2015[30]\end{array}$ & $\begin{array}{c}\text { Prospective, randomised, } \\
\text { blinded, placebo controlled/ } \\
\text { Keratoconus }\end{array}$ & 29 & 37 & $\begin{array}{c}\text { 1. } \uparrow \geq 1 \mathrm{D} \text { in Kmax } / 1 \text { year } \\
\text { 2. Clinically significant } \\
\Delta \text { refraction }\end{array}$ & -/Riboflavin $0.1 \%$ & $\begin{array}{l}\text { Corneal refractive power } \\
\text { decreased in treatment } \\
\text { group but increased in } \\
\text { control group. }\end{array}$ & $\begin{array}{c}\text { Kmax } \\
\text { (treatment): } \\
47.3 \pm 2.2 \\
\text { Kmax } \\
\text { (control): } 50.9 \\
\pm 5.7 \\
(\mathrm{P}=0.05)\end{array}$ & \begin{tabular}{|c|} 
Treatment: \\
$-0.35 \pm 0.58$ \\
Control: +0.11 \\
\pm 0.61 \\
$(\mathrm{P}=0.02)$
\end{tabular} & - & - & - \\
\hline
\end{tabular}


Acuity $C D V A=$ Corrected Distance Visual Acuity $\operatorname{Kmax}=$ maximum keratometry $\max =$ maximum $\min =\operatorname{minimum} K=$ keratometry $D=\operatorname{dioptre} c y l=$ cylinder $S E=$ spherical equivalent $M R S E=$ Manifest Refraction Spherical Equivalent $S D E=$ Snellen Decimal Equivalent $w=$ with $S I M=$ Simulated

Table 1B. Summary of outcomes for standard epithelium-off cross-linking. (Prospective non-randomised studies) $\left(3 \mathrm{~mW} / \mathrm{cm}^{2} \mathrm{UV}-\mathrm{A} \mathrm{exposure,} 5.4 \mathrm{~J} / \mathrm{cm}^{2}\right)$.

\begin{tabular}{|c|c|c|c|c|c|c|c|c|c|c|c|}
\hline Study & Study design/ & No. of & Follow-up, & Criteria for & UV device/UV energy/ Riboflavin & \multicolumn{6}{|c|}{ Outcome } \\
\hline- & - & - & - & - & - & Overall & $\begin{array}{l}\text { Pre-op K } \\
\text { (D) }\end{array}$ & $\Delta \mathrm{K}(\mathrm{D})$ & $\triangle \mathrm{UCVA}$ & $\triangle B C V A$ & $\begin{array}{c}\Delta \text { Refraction } \\
\text { (D) }\end{array}$ \\
\hline $\begin{array}{c}\text { Caporossi et al, } \\
2006[12]\end{array}$ & $\begin{array}{l}\text { Prospective non- } \\
\text { randomised open/ } \\
\text { Keratoconus }\end{array}$ & $\mid \begin{array}{c}18 ; 10 \\
\text { treated, } 8 \\
\text { FE } \\
\text { control }\end{array}$ & 6 & - & $\begin{array}{c}\text { Exerion-Sas/ Riboflavin } 0.1 \% \mathrm{w} \\
\text { dextran } 20 \%\end{array}$ & \begin{tabular}{|} 
Reduction in mean \\
spherical equivalent, \\
improvement in \\
morphologic symmetry \\
with reduction in \\
comatic aberrations.
\end{tabular} & - & $\begin{array}{c}\Delta \text { Mean } \mathrm{K} \\
\text { (treated): } \\
-2.1 \pm 0.13 \\
\text { in central } \\
3.0 \mathrm{~mm}\end{array}$ & $\begin{array}{c}\text { Treated: } 3.6 \\
\text { lines } \\
\text { improvement }(\mathrm{P} \\
=0.0000112)\end{array}$ & $\begin{array}{l}\text { Treated: } 1.66 \\
\text { lines } \\
\text { improvement } \\
\text { (Glasses) }(\mathrm{P}= \\
0.00071)\end{array}$ & $\begin{array}{c}\text { SE (treated): } \\
-2.5\end{array}$ \\
\hline
\end{tabular}




\begin{tabular}{|c|c|c|c|c|c|c|c|c|c|c|c|}
\hline \multirow{2}{*}{$\frac{\text { Study }}{-}$} & \multirow{2}{*}{$\begin{array}{c}\begin{array}{c}\text { Study design/ } \\
\text { Indication }\end{array} \\
-\end{array}$} & \multirow{2}{*}{$\begin{array}{c}\begin{array}{c}\text { No. of } \\
\text { Eyes }\end{array} \\
-\end{array}$} & \multirow{2}{*}{$\begin{array}{c}\begin{array}{c}\text { Follow-up, } \\
\text { months }\end{array} \\
-\end{array}$} & \multirow{2}{*}{$\begin{array}{c}\text { Criteria for } \\
\text { Progression } \\
-\end{array}$} & \multirow{2}{*}{\begin{tabular}{|c|} 
UV device/UV energy/ Riboflavin \\
- \\
\end{tabular}} & \multicolumn{6}{|c|}{ Outcome } \\
\hline & & & & & & Overall & $\begin{array}{l}\text { Pre-op K } \\
\text { (D) }\end{array}$ & $\Delta K(D)$ & $\triangle \mathrm{UCVA}$ & $\triangle B C V A$ & \begin{tabular}{|c|}
$\Delta$ Refraction \\
(D)
\end{tabular} \\
\hline $\begin{array}{l}\text { Vinciguerra et } \\
\text { al, 2009 [10] }\end{array}$ & $\begin{array}{l}\text { Prospective, non- } \\
\text { randomised single- } \\
\text { center/ Keratoconus }\end{array}$ & $\begin{array}{c}56 ; 28 \\
\text { treated, } \\
28 \mathrm{FE} \\
\text { control }\end{array}$ & 12 & \begin{tabular}{|c|} 
1. $\Delta$ myopia and/or \\
cyl of $\geq 3 \mathrm{D} / 6$ \\
months \\
2. Mean central $\Delta$ \\
$\mathrm{K} \geq 1.5 \mathrm{D}$ in 3 \\
consecutive \\
topographies/ 6 \\
months \\
3. Mean CCT $\downarrow \geq$ \\
$5 \%$ in 3 \\
consecutive \\
tomographies $/ 6$ \\
months \\
\end{tabular} & $\begin{array}{l}\text { Peschke Meditrade/ Riboflavin } \\
0.1 \% \text { w dextran } 20 \%\end{array}$ & \begin{tabular}{|} 
Improved UCVA and \\
BSCVA, reduced APP \\
and AK, reduced \\
corneal and total \\
wavefront aberrations.
\end{tabular} & $\begin{array}{c}\text { SIM K } \\
\text { steepest } \\
\text { (treated): } \\
50.37\end{array}$ & \begin{tabular}{|c} 
Treated: \\
$-6.16(\mathrm{P}<$ \\
$0.05)$
\end{tabular} & $\begin{array}{c}\text { Treated: From } \\
0.17 \pm 0.09 \text { to } \\
0.27 \pm 0.08 \\
\text { (LogMAR) }(\mathrm{P}< \\
0.05)\end{array}$ & \begin{tabular}{|c|} 
Treated: From \\
$0.52 \pm 0.17$ to \\
$0.72 \pm 0.16$ \\
(LogMAR) $(\mathrm{P}<$ \\
$0.05)$
\end{tabular} & $\begin{array}{c}\text { SE (treated): } \\
\text { From }-3.36 \pm \\
2.64 \text { to }-2.96 \pm \\
2.68\end{array}$ \\
\hline $\begin{array}{c}\text { Coskunseven } e t \\
a l, 2009[17]\end{array}$ & $\begin{array}{l}\text { Prospective } \\
\text { comparative/ } \\
\text { Keratoconus }\end{array}$ & $\begin{array}{l}38 ; 19 \\
\text { treated, } \\
19 \mathrm{FE} \\
\text { control }\end{array}$ & $5-12$ & $\begin{array}{c}\text { Increase in } \\
\text { maximum K by } 1 \mathrm{D} \\
/ 6 \text { months }\end{array}$ & $\begin{array}{l}\text { Peschke Meditrade/ Riboflavin } \\
0.1 \% \text { w dextran } 20 \%\end{array}$ & $\begin{array}{c}\text { Progression of } \\
\text { keratoconus stopped. } \downarrow \\
\text { in corneal curvature, } \mathrm{SE} \\
\text { refraction, and } \\
\text { refractive cylinder. }\end{array}$ & $\begin{array}{c}\text { Kmax } \\
\text { (treated): } \\
54.02 \pm \\
4.15 \\
\text { Kmax } \\
\text { (control): } \\
48.32 \pm \\
3.00\end{array}$ & $\begin{array}{c}\text { Treated: } \\
-1.57 \pm 1.14 \\
(\mathrm{P}<0.01) \\
\text { Control: }+ \\
0.04 \pm 1.34 \\
(\mathrm{P}=0.446)\end{array}$ & $\begin{array}{c}\text { Treated: } \\
\text { increased by } \\
0.06 \pm 0.05 \\
(\text { LogMAR) }(\mathrm{P}< \\
0.01) \\
\text { Control: } \\
\text { decreased by } \\
0.08 \pm 0.12 \\
\text { (LogMAR) }(\mathrm{P}< \\
0.1) \\
\end{array}$ & \begin{tabular}{|c|} 
Treated: increased \\
by $0.1 \pm 0.14$ \\
$($ LogMAR $)(\mathrm{P}<$ \\
$0.01)$ \\
Control: \\
decreased by 0.06 \\
\pm 0.09 (LogMAR) \\
$(\mathrm{P}<0.01)$
\end{tabular} & $\begin{array}{c}\mathrm{SE} \text { (treated): } \\
+1.03 \pm 2.22 \\
(\mathrm{P}<0.01) \\
\mathrm{SE}(\text { control): } \\
+0.03 \pm 0.96 \\
(\mathrm{P}=0.441)\end{array}$ \\
\hline $\begin{array}{l}\text { Vinciguerra et } \\
\text { al, 2009 [11] }\end{array}$ & $\begin{array}{l}\text { Prospective, } \\
\text { nonrandomized } \\
\text { single-center/ } \\
\text { Keratoconus }\end{array}$ & $\begin{array}{l}28 \\
\text { treated, } \\
28 \mathrm{FE} \\
\text { control }\end{array}$ & 24 & \begin{tabular}{|c|} 
Documented \\
keratoconus \\
progression in the \\
previous 6 months.
\end{tabular} & $\begin{array}{c}\text { Peschke } \\
\text { Meditrade } / 5.4 \mathrm{~J} / \mathrm{cm}^{2} / \text { Riboflavin } \\
0.1 \% \text { w dextran } 20 \%\end{array}$ & \begin{tabular}{|c|} 
Improved UCVA and \\
BSCVA, reduced APP \\
and AK, reduced \\
corneal and total \\
wavefront aberrations.
\end{tabular} & $\begin{array}{l}\text { Steepest } \\
\text { SIM K: } \\
50.37\end{array}$ & \begin{tabular}{|} 
SIM K: \\
50.37 to \\
$49.02(\mathrm{P}=$ \\
$0.03)$
\end{tabular} & $\begin{array}{c}\text { From } 0.77 \pm \\
0.18 \text { to } 0.53 \pm \\
0.19 \text { (LogMAR) }\end{array}$ & $\begin{array}{c}\text { From } 0.28 \pm 0.09 \\
\text { to } 0.13 \pm 0.10 \\
\text { (LogMAR) }\end{array}$ & $\begin{array}{c}\text { From }-3.37 \pm \\
2.64 \text { to }-2.56 \pm \\
2.68(\mathrm{P}= \\
0.03)\end{array}$ \\
\hline $\begin{array}{l}\text { Wollensak et } \\
a l, 2003[1]\end{array}$ & $\begin{array}{l}\text { Prospective, non- } \\
\text { randomised/ } \\
\text { Keratoconus }\end{array}$ & 23 & $\begin{array}{c}3-48 \\
\text { (Mean: } 23.2 \\
\pm 12.9)\end{array}$ & $\begin{array}{c}\text { Preoperative } \\
\text { progression of } \mathrm{K} \\
\text { value: } 1.42 \pm 1.18 \\
\mathrm{D} \text { in } 6 \text { months }\end{array}$ & $\begin{array}{l}\text { 370nm; Roithner Lasertechnik/ } \\
\text { Riboflavin } 0.1 \% \text { w dextran } 20 \%\end{array}$ & \begin{tabular}{|c|} 
Progression of \\
keratoconus stopped, \\
visual acuity improved \\
slightly.
\end{tabular} & $\begin{array}{l}\text { Max K: } \\
48-72\end{array}$ & $\begin{array}{c}-2.01 \pm 1.74 \\
(\mathrm{P}= \\
0.0001)\end{array}$ & NA & $\begin{array}{c}1.26 \pm 1.5(\mathrm{P}= \\
0.026)\end{array}$ & $\begin{array}{l}\text { SE: }-1.14 \pm \\
2.18(\mathrm{P}= \\
0.030)\end{array}$ \\
\hline
\end{tabular}




\begin{tabular}{|c|c|c|c|c|c|c|c|c|c|c|c|}
\hline Study & $\begin{array}{l}\text { Study design/ } \\
\text { Indication }\end{array}$ & $\begin{array}{l}\text { No. of } \\
\text { Eyes }\end{array}$ & $\begin{array}{c}\text { Follow-up, } \\
\text { months }\end{array}$ & $\begin{array}{l}\text { Criteria for } \\
\text { Progression }\end{array}$ & UV device/UV energy/ Riboflavin & \multicolumn{6}{|c|}{ Outcome } \\
\hline - & - & - & - & - & - & Overall & \begin{tabular}{|c|} 
Pre-op K \\
(D)
\end{tabular} & $\Delta K(D)$ & $\triangle \mathrm{UCVA}$ & $\triangle B C V A$ & $\begin{array}{l}\Delta \text { Refraction } \\
\text { (D) }\end{array}$ \\
\hline $\begin{array}{c}\text { Agrawal, } 2009 \\
{[15]}\end{array}$ & $\begin{array}{c}\text { Retrospective } \\
\text { nonrandomised open } \\
\text { label/ Keratoconus }\end{array}$ & 68 & $\begin{array}{c}6-16 \\
\text { (mean } \\
\text { follow-up: } \\
10.05 \pm \\
3.55)\end{array}$ & $\begin{array}{c}\text { 1. } \uparrow \text { max K of } 1.00 \\
\mathrm{D} / 1 \text { year } \\
\text { 2. Patient reports of } \\
\text { deteriorating } \\
\text { BCVA } \\
\text { 3. Need for new } \\
\text { contact lens fitting } \\
>\text { once } / 2 \text { years } \\
\end{array}$ & CBM X Linker/Riboflavin $0.1 \%$ & $\begin{array}{l}\text { BCVA improved } \\
\text { slightly, astigmatism } \\
\text { decreased, } K \text { value of } \\
\text { the apex decreased, } \\
\text { reduction in comatic } \\
\text { aberrations }\end{array}$ & \begin{tabular}{|c|} 
Mean $\max$ \\
K: $53.26 \pm$ \\
5.93
\end{tabular} & $\begin{array}{c}-2.47(54 \%) \\
(\mathrm{P}=0.004), \\
\text { stable } \\
(38 \%)\end{array}$ & - & $\begin{array}{c}1 \text { line } \\
\text { improvement } \\
(54 \%), \text { stable } \\
(28 \%)(\mathrm{P}=0.006)\end{array}$ & $\begin{array}{c}\text { Cyl: }-1.2 \pm \\
4.02\end{array}$ \\
\hline $\begin{array}{c}\text { Arbelaez et al, } \\
2009[16]\end{array}$ & $\begin{array}{l}\text { Prospective, } \\
\text { nonrandomized/ } \\
\text { Keratoconus }\end{array}$ & 20 & 12 & \begin{tabular}{|c|}
$1 . \uparrow$ maximum $K$ \\
readings in several \\
measurements over \\
$3-6$ months \\
2. Changes in \\
refraction \\
3. $\downarrow$ visual acuity \\
and contact lens \\
intolerance
\end{tabular} & UV-X device/ Riboflavin & $\begin{array}{l}\text { Improved UCVA and } \\
\text { BSCVA. Reduced } \\
\text { average keratometry } \\
\text { reading, manifest } \\
\text { refraction sphere and } \\
\text { manifest cyl }\end{array}$ & \begin{tabular}{|c|} 
Kmax \\
apex: 51.89 \\
\pm 7.99
\end{tabular} & $\begin{array}{c}-1.4(\mathrm{P}= \\
0.01)\end{array}$ & $\begin{array}{l}4.15 \text { line } \\
\text { improvement }(\mathrm{P} \\
=0.002)\end{array}$ & $\begin{array}{c}1.65 \text { line } \\
\text { improvement }(P= \\
0.002)\end{array}$ & $\begin{array}{c}\text { Sphere: }-1.26 \\
(\mathrm{P}=0.0033) \\
\text { Cyl: }-1.25(\mathrm{P} \\
=0.0003)\end{array}$ \\
\hline
\end{tabular}

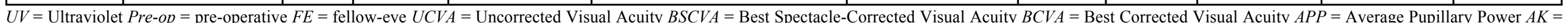
Apical Keratometry $\operatorname{Kmax}=$ maximum keratometry $\max =\operatorname{maximum} K=$ keratometry $D=$ Dioptre $c y l=$ cylinder $C C T=$ Central Corneal Thickness $S E=$ Spherical Equivalent $w=$ with $S I M=$ simulated

Table 1C. Summary of outcomes for standard epithelium-off cross-linking. (Case series) $\left(3 \mathrm{~mW} / \mathrm{cm}^{2} \mathrm{UVA}\right.$ exposure, $\left.5.4 \mathrm{~J} / \mathrm{cm}^{2}\right)$.

\begin{tabular}{|c|c|c|c|c|c|c|c|c|c|c|c|}
\hline Study & $\begin{array}{l}\text { Study design/ } \\
\text { Indication }\end{array}$ & $\begin{array}{c}\text { No. } \\
\text { of }\end{array}$ & $\begin{array}{l}\text { Follow-up, } \\
\text { months }\end{array}$ & Criteria for Progression & $\begin{array}{c}\text { UV device/ UV } \\
\text { energy/ Riboflavin }\end{array}$ & \multicolumn{6}{|c|}{ Outcome } \\
\hline- & - & - & - & - & - & Overall & $\begin{array}{l}\text { Pre-op K } \\
\text { (D) }\end{array}$ & $\Delta K(D)$ & $\triangle \mathrm{UCVA}$ & $\triangle \mathrm{BCVA}$ & $\begin{array}{l}\Delta \text { Refraction } \\
\text { (D) }\end{array}$ \\
\hline $\begin{array}{l}\text { Vinciguerra et } \\
a l, 2012[31]\end{array}$ & $\begin{array}{c}\text { Prospective, } \\
\text { interventional case } \\
\text { series/ Keratoconus }\end{array}$ & 40 & 24 & $\begin{array}{c}\text { Documented keratoconus } \\
\text { progression in the } \\
\text { previous } 3 \text { months }\end{array}$ & $\begin{array}{c}\text { CSO-VEGA X- } \\
\text { linker/ Riboflavin } \\
0.1 \% \text { w dextran } 20 \%\end{array}$ & $\begin{array}{l}\text { Improved UCVA and } \\
\text { BSCVA. Reduced corneal } \\
\text { asymmetry and total } \\
\text { wavefront aberrations }\end{array}$ & $\begin{array}{c}\text { SIM K } \\
\text { Steepest: } \\
51.48 \pm 3.4\end{array}$ & $\begin{array}{c}\text { From } 51.48 \\
\pm 3.4 \text { to } \\
50.21 \pm 3.2 \\
(\mathrm{P}=0.07)\end{array}$ & $\begin{array}{c}\text { From } 0.79 \pm \\
0.21 \text { to } 0.58 \pm \\
0.18(\text { LogMAR }) \\
(\mathrm{P}<0.05)\end{array}$ & $\begin{array}{c}\text { From } 0.39 \pm 0.10 \\
\text { to } 0.20 \pm 0.09 \\
(\text { LogMAR) }(\mathrm{P}< \\
0.05)\end{array}$ & $\begin{array}{c}\text { Mean SE: }-1.57 \\
(P=0.02)\end{array}$ \\
\hline
\end{tabular}




\begin{tabular}{|c|c|c|c|c|c|c|c|c|c|c|c|}
\hline \multirow{2}{*}{$\begin{array}{c}\text { Study } \\
-\end{array}$} & \multirow{2}{*}{$\begin{array}{c}\text { Study design/ } \\
\text { Indication }\end{array}$} & \multirow{2}{*}{\begin{tabular}{|c|}
$\begin{array}{c}\text { No. } \\
\text { of } \\
\text { Eyes }\end{array}$ \\
- \\
\end{tabular}} & \multirow{2}{*}{\begin{tabular}{|c|}
$\begin{array}{c}\text { Follow-up, } \\
\text { months }\end{array}$ \\
- \\
\end{tabular}} & \multirow{2}{*}{\begin{tabular}{|c|} 
Criteria for Progression \\
- \\
\end{tabular}} & \multirow{2}{*}{$\begin{array}{c}\begin{array}{c}\text { UV device/ UV } \\
\text { energy/ Riboflavin }\end{array} \\
-\end{array}$} & \multicolumn{6}{|c|}{ Outcome } \\
\hline & & & & & & Overall & $\begin{array}{l}\text { Pre-op K } \\
\text { (D) }\end{array}$ & $\Delta K(D)$ & $\triangle \mathrm{UCVA}$ & $\triangle B C V A$ & $\begin{array}{l}\Delta \text { Refraction } \\
\text { (D) }\end{array}$ \\
\hline $\begin{array}{l}\text { Vinciguerra } e t \\
a l, 2010[27]\end{array}$ & $\begin{array}{c}\text { Prospective case series/ } \\
\text { Post-laser ectasia }\end{array}$ & 13 & 12 & \begin{tabular}{|c|}
$1 . \Delta$ in myopia/ \\
astigmatism of $\geq 3 \mathrm{D} / 6$ \\
months \\
2. Mean $\Delta$ in central \\
and/or pupillary $\mathrm{K} \geq$ \\
$1.50 \mathrm{D}$ in 3 consecutive \\
topographies $/ 6$ months \\
3. Total mean CCT $\downarrow$ of $\geq$ \\
$5 \%$ in 3 consecutive \\
tomographies $/ 6$ months
\end{tabular} & $\begin{array}{l}\text { Peschke Meditrade/ } \\
\text { Riboflavin } 0.1 \% \mathrm{w} \\
\text { dextran } 20 \%\end{array}$ & \begin{tabular}{|c|} 
Improved BSCVA. \\
Reduced mean SE \\
refraction and mean \\
refractive sphere reduction.
\end{tabular} & $\begin{array}{c}\text { SIM K } \\
\text { steepest: } \\
45.93 \pm 6.03\end{array}$ & $\begin{array}{c}\text { From } 45.93 \\
\pm 6.03 \text { to } \\
42.49 \pm 4.88 \\
(\mathrm{P}>0.05)\end{array}$ & $\begin{array}{c}\text { From } 1.08 \pm \\
0.43 \text { to } 0.94 \pm \\
0.46(\text { LogMAR }) \\
(\mathrm{P}>0.05)\end{array}$ & $\begin{array}{c}\text { From } 0.16 \pm 0.14 \\
\text { to } 0.06 \pm 0.08 \\
\text { (LogMAR) }(\mathrm{P}< \\
0.05)\end{array}$ & $\begin{array}{c}\text { SE: From }-4.16 \\
\pm 2.90 \text { to }-3.25 \\
\pm 2.05(\mathrm{P}> \\
0.05)\end{array}$ \\
\hline $\begin{array}{l}\text { Salgado et al, } \\
2011[26]\end{array}$ & $\begin{array}{c}\text { Prospective case series/ } \\
\text { Post-laser ectasia }\end{array}$ & 22 & 12 & $\begin{array}{l}\text { Progressive keratectasia } \\
\text { after refractive surgery }\end{array}$ & $\begin{array}{c}\text { Peschke Meditrade/ } \\
\text { Riboflavin } 0.1 \% \mathrm{w} \\
\text { dextran } 20 \%\end{array}$ & $\begin{array}{c}\text { Improved BCVA, UCVA } \\
\text { and max-K. }\end{array}$ & $\begin{array}{c}\text { Max K: } \\
44.12 \pm 3.97\end{array}$ & $\begin{array}{c}\text { From } 44.12 \\
\pm 3.97 \text { to } \\
44.43 \pm 4.06 \\
(\mathrm{P}>0.05)\end{array}$ & $\begin{array}{c}\text { From } 0.53 \pm \\
0.38 \text { to } 0.40 \pm \\
0.27(\text { LogMAR }) \\
(\mathrm{P}>0.05)\end{array}$ & $\begin{array}{c}\text { From } 0.19 \pm 0.21 \\
\text { to } 0.15 \pm 0.14 \\
\text { (LogMAR) }(\mathrm{P}> \\
0.05)\end{array}$ & $\begin{array}{c}\text { SE: From }-2.39 \\
\pm 2.03 \text { to }-2.07 \\
\pm 2.18 \\
\text { (LogMAR) (P > } \\
0.05)\end{array}$ \\
\hline $\begin{array}{l}\text { Ivarsen et al, } \\
2013[32]\end{array}$ & $\begin{array}{l}\text { Retrospective follow- } \\
\text { up/ Keratoconus }\end{array}$ & 28 & 22 & $\begin{array}{c}\text { 1. } \uparrow \operatorname{max~} \mathrm{K} \geq 1.5 \mathrm{D} / 3-6 \\
\text { months } \\
\text { 2. } \downarrow \text { vision and } \Delta \\
\text { refraction }\end{array}$ & $\begin{array}{l}\text { IROC UV-X/ } \\
\text { Riboflavin } 0.1 \% \mathrm{w} \\
\text { dextran } 20 \%\end{array}$ & $\begin{array}{l}\text { Progression of keratoconus } \\
\text { stopped, decreased max K. }\end{array}$ & $\begin{array}{c}\text { Max K: } 61.2 \\
\pm 3.7\end{array}$ & $\begin{array}{c}\text { From } 61.2 \pm \\
3.7 \text { to } 59.1 \pm \\
3.7\end{array}$ & - & Unchanged & - \\
\hline $\begin{array}{l}\text { Richoz et al, } \\
2013 \text { [29] }\end{array}$ & $\begin{array}{c}\text { Retrospective, } \\
\text { interventional case } \\
\text { series/Post-laser ectasia }\end{array}$ & 26 & $\begin{array}{c}12-62 \text { (mean } \\
\text { follow-up: } 25 \\
\pm 13 \text { ) }\end{array}$ & $\begin{array}{c}\uparrow \text { Kmax of anterior } \\
\text { corneal surface, at } 3.0 \mathrm{~mm} \\
\text { from apex of } \geq 1 \mathrm{D} / 12 \\
\text { months }\end{array}$ & $\begin{array}{c}\text { Peschke Meditrade/ } \\
\text { Riboflavin } 0.1 \% \mathrm{w} \\
\text { dextran } 20 \%\end{array}$ & \begin{tabular}{|} 
Improved mean CDVA, \\
reduced mean Kmax. \\
Significantly reduced index \\
of surface variance, index \\
of vertical asymmetry, \\
keratoconus index and \\
central keratoconus index
\end{tabular} & $\begin{array}{c}\text { Mean Kmax: } \\
52.8 \pm 5\end{array}$ & $\begin{array}{c}-1.9 \pm 1.9(\mathrm{P} \\
<0.001)\end{array}$ & - & $\begin{array}{c}\text { From } 0.5 \pm 0.3 \text { to } \\
0.2 \pm 0.16 \\
\text { (LogMAR) }(\mathrm{P}< \\
0.001)\end{array}$ & - \\
\hline
\end{tabular}

$U V=$ Ultraviolet. $U C V A=$ Uncorrected Visual Acuity. $B S C V A=$ Best Spectacle-Corrected Visual Acuity. $B C V A=$ Best Corrected Visual Acuity.

$C D V A=$ Corrected Distance Visual Acuity. Kmax $=$ maximum keratometry. $\max =$ maximum. $K=$ Keratometry $. D=$ Dioptre.

$C C T=$ Central Corneal Thickness. $S E=$ Spherical Equivalent. pre-op $=$ pre-operative. $w=$ with $S I M=$ simulated. 




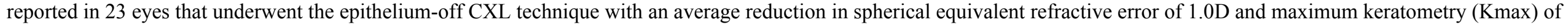


Comparative studies using the fellow eye as control showed stabilization of the treated eye and continued progression in the fellow untreated eye [17].

Wittig-Silva et al performed the first randomized controlled trial and found significant flattening of the steepest keratometry and a trend towards better visual acuity with long-

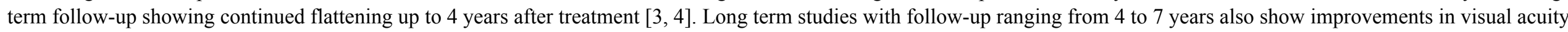

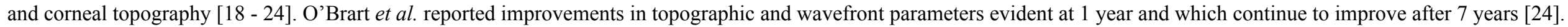

Table 2. Summary of outcomes for long-term studies of standard epithelium-off cross-linking.

\begin{tabular}{|c|c|c|c|c|c|c|c|c|c|c|c|}
\hline Study & Study design/ & No. of & Follow-up, & Criteria for & UV device/ & \multicolumn{6}{|c|}{ Outcome } \\
\hline - & - & - & - & - & - & Overall & $\begin{array}{l}\text { Pre-op K } \\
\text { (D) }\end{array}$ & $\Delta \mathrm{K}$ (D) & $\Delta$ UCVA & $\triangle$ BCVA & $\Delta$ Refraction (D) \\
\hline $\begin{array}{c}\text { Caporossi et al, } \\
2010[21]\end{array}$ & $\begin{array}{c}\text { Prospective, } \\
\text { nonrandomised, open } \\
\text { long-term trial/ } \\
\text { Keratoconus }\end{array}$ & $\begin{array}{c}88 ; 44 \\
\text { treated, } \\
44 \mathrm{FE} \\
\text { control }\end{array}$ & 48 & - & $\begin{array}{c}\text { CSO Vega CBM } \\
\text { X linker/ } \\
\text { Riboflavin } 0.1 \% \mathrm{w} \\
\text { dextran } 20 \%\end{array}$ & $\begin{array}{l}\text { Reduced mean } \mathrm{K} \text { value, } \\
\text { reduced coma aberration. } \\
\text { Improved BSCVA and } \\
\text { UCVA. }\end{array}$ & - & \begin{tabular}{|c|} 
Mean K \\
(treated): \\
$-2.26 \pm 0.68$ \\
Mean K (FE \\
control): +2.2 \\
\pm 1.24 \\
\end{tabular} & $\begin{array}{c}\text { Treated: }+2.85 \pm \\
0.81 \text { (Snellen } \\
\text { lines) }\end{array}$ & \begin{tabular}{|c|} 
Treated: $+2.03 \pm$ \\
1.04 (Snellen \\
lines)
\end{tabular} & $\begin{array}{c}\text { SE (treated): } \\
+2.15 \pm 1.19(\mathrm{P}= \\
5.1 \times 10^{-10}\end{array}$ \\
\hline $\begin{array}{l}\text { O'Brart et al, } \\
2015[24]\end{array}$ & $\begin{array}{l}\text { Prospective cohort } \\
\text { study/Keratoconus }\end{array}$ & $\begin{array}{l}65 ; 36 \\
\text { treated, } \\
29 \mathrm{FE} \\
\text { control }\end{array}$ & 84 & $\mid \begin{array}{c}\text { 1. } \downarrow \text { UDVA/ CDVA } \\
\text { by }>1 \text { line } \\
\text { 2. deteriorating } \\
\text { refractive/ corneal } \\
\text { astigmatism, SIM K/ } \\
\text { Kmax by } \\
0.75 \mathrm{D} / 12-24 \text { months }\end{array}$ & $\begin{array}{c}-/ \text { Riboflavin } 0.1 \% \\
\text { w dextran } 20 \%\end{array}$ & $\begin{array}{c}\text { Improvements in } \\
\text { topographic and wavefront } \\
\text { parameters evident at } 1 \\
\text { year continue to improve } \\
\text { after } 7 \text { years. }\end{array}$ & $\begin{array}{c}\text { Mean Kmax } \\
\text { (treated): } \\
48.23 \pm 3.49 \\
\text { Mean Kmax } \\
\text { (FE } \\
\text { control): } \\
47.01 \pm 3.54\end{array}$ & $\begin{array}{c}\text { Treated: }-0.91 \\
(\mathrm{P}<0.001) \\
\text { FE control: } \\
+0.86(\mathrm{P}< \\
0.05)\end{array}$ & \begin{tabular}{|c|} 
Treated: From \\
$0.32 \pm 0.26$ to \\
$0.46 \pm 0.5$ (SDE) \\
$(\mathrm{P}<0.0005)$ \\
FE control: From \\
$0.56 \pm 0.4$ to 0.43 \\
\pm 0.37 (SDE) $(\mathrm{P}$ \\
$=0.4)$
\end{tabular} & \begin{tabular}{|c|} 
Treated: From \\
$0.85 \pm 0.25$ to \\
$0.96 \pm 0.17$ (SDE) \\
$(\mathrm{P}<0.0001)$ \\
FE control: $0.91 \pm$ \\
0.28 to $0.92 \pm$ \\
0.29 (SDE) $(\mathrm{P}=$ \\
$0.9)$ \\
\end{tabular} & $\begin{array}{c}\text { Mean SE } \\
\text { (treated): }+0.78 \text { (P } \\
<0.005) \\
\text { Mean SE (FE } \\
\text { control): }-1.66 \pm \\
2.51 \text { to }-1.72 \pm \\
2.27(\mathrm{P}=0.8)\end{array}$ \\
\hline $\begin{array}{l}\text { O'Brart et al, } \\
2013 \text { [19] }\end{array}$ & $\begin{array}{l}\text { Follow-up study/ } \\
\text { Keratoconus }\end{array}$ & 30 & $48-72$ & \begin{tabular}{|c|}
$1 . \downarrow$ UDVA/ CDVA \\
by $>1$ line \\
$2 . \downarrow$ refractive/ \\
corneal astigmatism, \\
$\mathrm{K}$ or cone apex \\
power by \\
$0.75 \mathrm{D} / 12-24$ months
\end{tabular} & $\begin{array}{c}\text {-/Riboflavin } 0.1 \% \\
\text { w dextran } 20 \%\end{array}$ & $\begin{array}{l}\text { Reduced mean spherical } \\
\text { equivalent, mean simulated } \\
\text { K, cone apex power. } \\
\text { Improved CDVA. }\end{array}$ & \begin{tabular}{|c} 
Mean SIM \\
K: $46.44 \pm$ \\
3.4
\end{tabular} & $\mid \begin{array}{c}\text { From } 46.44 \pm \\
3.4 \text { to } 45.6 \pm \\
3.3(\mathrm{P}< \\
0.001)\end{array}$ & \begin{tabular}{|c|} 
From $+0.27 \pm$ \\
0.29 to $+0.286 \pm$ \\
$0.31(\mathrm{SDE})(\mathrm{P}=$ \\
$0.6)$
\end{tabular} & $\begin{array}{c}\text { From } 0.8 \pm 0.27 \text { to } \\
0.905 \pm 0.2(\mathrm{SDE}) \\
(\mathrm{P}<0.04)\end{array}$ & \begin{tabular}{|l} 
SE: From $-1.61 \pm$ \\
1.97 to $-0.79 \pm$ \\
$1.7(\mathrm{P}<0.001)$
\end{tabular} \\
\hline
\end{tabular}




\begin{tabular}{|c|c|c|c|c|c|c|c|c|c|c|c|}
\hline Study & Study design/ & $\begin{array}{l}\text { No. of } \\
\text { Eves }\end{array}$ & $\begin{array}{l}\text { Follow-up, } \\
\text { months }\end{array}$ & $\begin{array}{l}\text { Criteria for } \\
\text { Progression }\end{array}$ & $\begin{array}{l}\text { UV device/ } \\
\text { Riboflavin }\end{array}$ & \multicolumn{6}{|c|}{ Outcome } \\
\hline- & - & - & - & - & - & Overall & $\begin{array}{l}\text { Pre-op K } \\
\text { (D) }\end{array}$ & $\Delta \mathrm{K}(\mathrm{D})$ & $\triangle$ UCVA & $\triangle \mathrm{BCVA}$ & $\Delta$ Refraction (D) \\
\hline $\begin{array}{l}\text { Hashemi et al, } \\
2013 \text { [20] }\end{array}$ & $\begin{array}{c}\text { Prospective case series/ } \\
\text { Keratoconus }\end{array}$ & 40 & 60 & \begin{tabular}{|c|}
$1 . \uparrow \geq 1 \mathrm{D}$ in max $\mathrm{K}$ \\
manifest cyl error or \\
MRSE \\
2. $\downarrow \geq 2$ lines of \\
BCVA
\end{tabular} & $\begin{array}{c}\text { UV-X IROC/ } \\
\text { Riboflavin } 0.1 \% \mathrm{w} \\
\text { dextran } 20 \%\end{array}$ & $\begin{array}{c}\text { Improved BCVA. No } \\
\text { change in mean K and max } \\
\text { K, UCVA, and } \\
\text { astigmatism. }\end{array}$ & $\begin{array}{c}\text { Max K: } \\
49.37 \pm 3.48\end{array}$ & $\begin{array}{c}\text { From } 49.37 \pm \\
3.48 \text { to } 49.13 \\
\pm 3.29(\mathrm{P}= \\
0.645)\end{array}$ & $\begin{array}{c}\text { From } 0.67 \pm 0.52 \\
\text { to } 0.65 \pm 0.51 \\
\text { (LogMAR) }(\mathrm{P}= \\
0.853)\end{array}$ & $\begin{array}{c}\text { From } 0.31 \pm 0.28 \\
\text { to } 0.19 \pm 0.20 \\
\text { (LogMAR) }(\mathrm{P}= \\
0.016)\end{array}$ & \begin{tabular}{|c|} 
Mean MRSE: \\
From $-3.18 \pm 2.23$ \\
to $-2.77 \pm 2.18(\mathrm{P}$ \\
$=0.174)$
\end{tabular} \\
\hline $\begin{array}{l}\text { Ucakhan et al, } \\
2016[22]\end{array}$ & $\begin{array}{l}\text { Prospective follow-up } \\
\text { study/Keratoconus }\end{array}$ & 40 & 48 & $\begin{array}{c}\uparrow>1 \mathrm{D} \text { in } \mathrm{Kmax} / 12 \\
\text { months }\end{array}$ & \begin{tabular}{|c|} 
UV-X, IROC/ \\
Riboflavin $0.1 \% \mathrm{w}$ \\
dextran $20 \%$
\end{tabular} & $\begin{array}{c}\text { Improved UCVA and } \\
\text { BSCVA. Reduced mean } \\
\text { Kmax. }\end{array}$ & \begin{tabular}{|c|} 
Mean \\
Kmax: 58.4 \\
\pm 5.5
\end{tabular} & $\begin{array}{l}-1.2 \pm 2.2(\mathrm{P} \\
=0.0046)\end{array}$ & $\begin{array}{c}-0.4 \pm 0.2 \\
(\operatorname{LogMAR})(\mathrm{P}= \\
0.0001)\end{array}$ & $\begin{array}{c}-0.2 \pm 0.2 \\
(\text { LogMAR) }(\mathrm{P}= \\
0.0001)\end{array}$ & \begin{tabular}{|c|} 
MRSE: From -6.2 \\
\pm 3.5 to $-5.4 \pm 3.8$ \\
$(\mathrm{P}=0.04)$
\end{tabular} \\
\hline $\begin{array}{c}\text { Raiskup-Wolf } e t \\
a l, 2008[18]\end{array}$ & $\begin{array}{c}\text { Long-term retrospective } \\
\text { study/ Keratoconus }\end{array}$ & 241 & Max 72 & \begin{tabular}{c|}
$1 . \uparrow \max \mathrm{K} 1 \mathrm{D} / 1$ \\
year \\
2. $\downarrow$ visual acuity \\
3. New CL/2 years
\end{tabular} & $\begin{array}{c}\text { Fa. Peschke/ } \\
\text { Riboflavin } 0.1 \%\end{array}$ & $\begin{array}{l}\text { Reduction in steepening, } \\
\text { improved BCVA }\end{array}$ & $\begin{array}{c}\text { Kmax: } 53.7 \\
\pm 7.5\end{array}$ & $-2.57 \pm 3.71$ & - & $-0.15 \pm 0.18$ & - \\
\hline $\begin{array}{l}\text { Raiskup et al, } \\
2015 \text { [23] }\end{array}$ & $\begin{array}{c}\text { Retrospective } \\
\text { interventional case } \\
\text { series/ Keratoconus }\end{array}$ & 34 & $\begin{array}{l}132 \text { (Mean: } \\
131.9 \pm \\
20.1)\end{array}$ & $\begin{array}{c}\uparrow \text { apical } \mathrm{K} \geq \\
1 \mathrm{D} / 6-12 \text { months }\end{array}$ & - & $\begin{array}{l}\text { Reduced AK value, max K } \\
\text { and min K. Improved } \\
\text { CDVA. ECC is unchanged. }\end{array}$ & - & - & - & $\begin{array}{c}-0.14 \text { (LogMAR) } \\
(\mathrm{P}=0.002)\end{array}$ & - \\
\hline
\end{tabular}

$U V=$ Ultraviolet pre-op $=$ pre-operative UCVA = Uncorrected Visual Acuity BSCVA = Best Spectacle-Corrected Visual Acuity

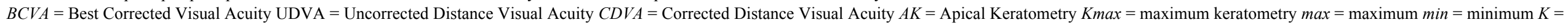
keratometry $E C C=$ Endothelial Cell Count $S D E=$ Snellen Decimal Equivalent

$D=$ diopters $F E=$ fellow eye $w=$ with $S E=$ spherical equivalent $S I M=$ simulated $c y l=$ cylinder

$M R S E=$ Manifest Refraction Spherical Equivalent $C L=$ Contact Lens

Epithelium-off CXL has also been shown to be effective in stabilizing post-LASIK ectasia with improvements in the visual acuity with more than 2 years follow-up [25 - 27].

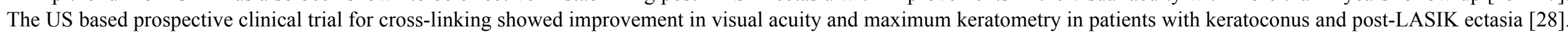

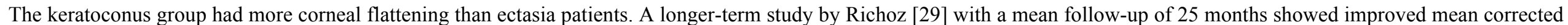
visual acuity and mean Kmax.

\section{PAEDIATRIC KERATOCONUS}

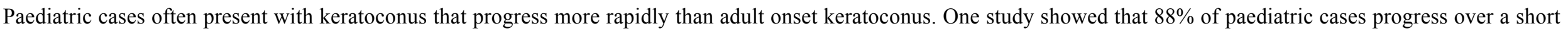

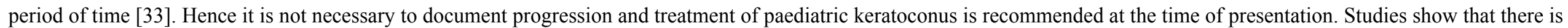



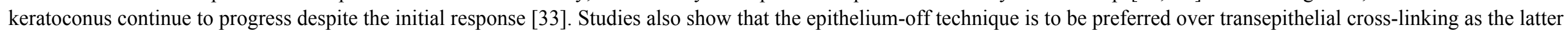
technique show worsening keratometry values over time [36]. 


\section{EPITHELIUM-ON (TRANSEPITHELIAL) CORNEAL CROSS-LINKING (TABLES 3A and 3B)}

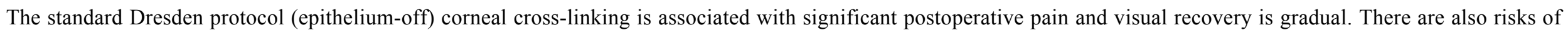

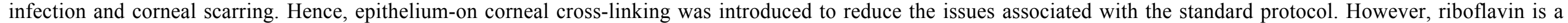



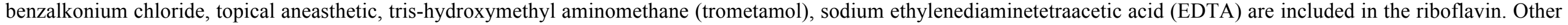
techniques include increased riboflavin concentration and iontophoresis.

The outcomes for transepithelial cross-linking using riboflavin with trometamol/EDTA are mixed. Some studies report improvement in visual acuity and keratometry

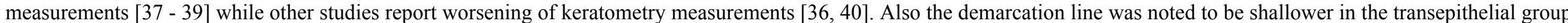
$[37]$.

Table 3A. Summary of outcomes for epithelium-on (transepithelial) cross-linking (Adults).

\begin{tabular}{|c|c|c|c|c|c|c|c|c|c|c|c|}
\hline Study & $\begin{array}{l}\text { Study design/ } \\
\text { Protocol }\end{array}$ & $\begin{array}{c}\text { No. of } \\
\text { Eyes }\end{array}$ & $\begin{array}{c}\text { Follow-up, } \\
\text { months }\end{array}$ & $\begin{array}{l}\text { Criteria for } \\
\text { Progression }\end{array}$ & $\begin{array}{c}\text { UV device/ UV energy/ } \\
\text { Riboflavin }\end{array}$ & \multicolumn{6}{|c|}{ Outcome } \\
\hline - & - & - & - & - & - & Overall & $\begin{array}{l}\text { Pre-op K } \\
\text { (D) }\end{array}$ & $\Delta K(D)$ & $\triangle \mathrm{UCVA}$ & $\triangle B C V A$ & $\begin{array}{l}\Delta \text { Refraction } \\
\text { (D) }\end{array}$ \\
\hline $\begin{array}{c}\text { Soeters et al, } \\
2015 \text { [42] }\end{array}$ & $\begin{array}{l}\text { Randomised clinical } \\
\text { trial } / 3 \mathrm{~mW} / \mathrm{cm}^{2} 30 \mathrm{~min}\end{array}$ & $\begin{array}{l}61 ; 35 \\
\text { epi-on, } \\
26 \text { epi- } \\
\text { off }\end{array}$ & 12 & $\begin{array}{c}\uparrow \mathrm{Kmax}, \text { Ksteep, } \\
\text { mean } \mathrm{K} \text { and } / \text { or } \\
\text { topographic cyl } \\
\text { value by } \geq \\
0.5 \mathrm{D} / 6-12 \text { months }\end{array}$ & \begin{tabular}{|c|} 
For both: UV-X; Peschke \\
Meditrade \\
Epi-on: $0.1 \%$ riboflavin \\
with $15.0 \%$ dextran, \\
trometamol and EDTA \\
Epi-off: isotonic \\
riboflavin $0.1 \%$ solution \\
with $20 \%$ dextran
\end{tabular} & $\begin{array}{l}\text { Average Kmax remained stable for } \\
\text { the epi-off group but showed } \\
\text { significant flattening in the epi-off } \\
\text { group. CDVA showed a better } \\
\text { outcome in the epi-on group. }\end{array}$ & $\begin{array}{c}\text { Kmax (epi- } \\
\text { off): } 57.8 \pm \\
7.1 \\
\text { Kmax (epi- } \\
\text { on): } 56.4 \pm \\
5.0\end{array}$ & $\mid \begin{array}{c}\text { Epi-off: }-1.5 \\
\pm 2.0 \\
\text { Epi-on: }+0.3 \\
\pm 1.8 \\
(\mathrm{P}=0.022)\end{array}$ & $\begin{array}{c}\text { Epi-off: }-0.15 \\
\pm 0.43 \\
\text { (LogMAR) } \\
\text { Epi-on: }-0.06 \\
\pm 0.37 \\
\text { (LogMAR) } \\
\text { (P=0.591) }\end{array}$ & \begin{tabular}{|c|} 
Epi-off: $-0.07 \pm$ \\
0.21 (LogMAR) \\
Epi-on: $-0.14 \pm$ \\
0.21 (LogMAR) \\
$(\mathrm{P}=0.023)$
\end{tabular} & $\begin{array}{c}\text { SE (Epi-off): } \\
+0.4 \pm 3.0 \\
\text { SE (Epi-on): } \\
+0.3 \pm 1.6 \\
(\mathrm{P}=0.436)\end{array}$ \\
\hline $\begin{array}{l}\text { Al Fayez et al, } \\
2015[41]\end{array}$ & $\begin{array}{l}\text { Prospective clinical } \\
\text { trial } / 3 \mathrm{~mW} / \mathrm{cm}^{2} 30 \mathrm{~min}\end{array}$ & $\begin{array}{l}70 ; 34 \\
\text { epi-on, } \\
36 \text { epi- } \\
\text { off }\end{array}$ & 36 & $\begin{array}{c}\uparrow \max \mathrm{K} / \text { manifest } \\
\text { astigmatism } \geq 1 \mathrm{D} / 12 \\
\text { months }\end{array}$ & $\begin{array}{c}\text { Epi-on: IROC/ } 1 \% \\
\text { tetracaine/ } 0.02 \% \\
\text { benzalkonium chloride, } \\
\text { dextran-free riboflavin } \\
\text { Epi-off: IROC/ } 0.1 \% \\
\text { riboflavin with dextran } \\
20 \% \text { solution } 30 \text { min }\end{array}$ & $\begin{array}{c}\text { Kmax decreased in the epi-off } \\
\text { group but increased in epi-on group. }\end{array}$ & - & $\begin{array}{c}\text { Kmax (epi- } \\
\text { off): }-2.4 \\
\text { Kmax (epi- } \\
\text { on): }+1.1 \\
(\mathrm{P}<0.0001)\end{array}$ & $\begin{array}{c}\text { Epi-off: }-0.2 \\
\text { (LogMAR) } \\
\text { Epi-on: }+0.1 \\
\text { (LogMAR) (P } \\
<0.0001)\end{array}$ & $\begin{array}{l}\text { Epi-off: }-0.1 \\
\text { (LogMAR) } \\
\text { Epi-on: }+0.06 \\
\text { (LogMAR) } \\
(\mathrm{P}=0.055)\end{array}$ & - \\
\hline
\end{tabular}




\begin{tabular}{|c|c|c|c|c|c|c|c|c|c|c|c|}
\hline Study & $\begin{array}{l}\text { Study design/ } \\
\text { Protocol }\end{array}$ & $\begin{array}{l}\text { No. of } \\
\text { Eyes }\end{array}$ & \begin{tabular}{|c|}
$\begin{array}{c}\text { Follow-up, } \\
\text { months }\end{array}$ \\
\end{tabular} & $\begin{array}{l}\text { Criteria for } \\
\text { Progression }\end{array}$ & $\begin{array}{c}\text { UV device/ UV energy/ } \\
\text { Riboflavin }\end{array}$ & \multicolumn{6}{|c|}{ Outcome } \\
\hline- & - & - & - & - & - & Overall & $\begin{array}{l}\text { Pre-op K } \\
\text { (D) }\end{array}$ & $\Delta K(D)$ & $\triangle \mathrm{UCVA}$ & $\triangle B C V A$ & \begin{tabular}{|l|}
$\Delta$ Refraction \\
(D)
\end{tabular} \\
\hline $\begin{array}{c}\text { Filippello et al, } \\
2012 \text { [37] }\end{array}$ & $\begin{array}{l}\text { Prospective case- } \\
\text { control cohort study/ } \\
3 \mathrm{~mW} / \mathrm{cm}^{2} 30 \mathrm{~min}\end{array}$ & $\begin{array}{l}40 ; 20 \\
\text { epi-on, } \\
20 \mathrm{FE} \\
\text { control }\end{array}$ & 18 & \begin{tabular}{|c} 
1. $\uparrow$ max cone apex \\
curvature $\geq 1 \mathrm{D} / 6$ \\
months \\
$2 . \downarrow$ corneal \\
thickness $>2 \% / 6$ \\
months \\
3. $\uparrow$ central corneal \\
astigmatism $\geq 1 \mathrm{D} / 6$ \\
months
\end{tabular} & $\begin{array}{l}\text { Vega/ } 0.1 \% \text { riboflavin } \\
\text { with dextrane T500, } \\
\text { trometamol and EDTA } \\
\text { sodium salt }\end{array}$ & $\begin{array}{l}\text { Improved UCVA and CVA, } \\
\text { topography-derived keratometry, } \\
\text { cone apex power, and HOA. }\end{array}$ & $\begin{array}{c}\text { SIM K } \\
\text { steepest } \\
\text { (treated): } \\
51.02 \pm 1.10 \\
\text { SIM K } \\
\text { steepest (FE } \\
\text { control): } \\
51.12 \pm 1.02\end{array}$ & \begin{tabular}{|c|} 
Treated: \\
From 51.02 \\
\pm 1.10 to \\
$48.05 \pm 0.21$ \\
FE control: \\
$51.12 \pm 1.02$ \\
to $52.12 \pm$ \\
0.47 \\
$(\mathrm{P}<0.05)$
\end{tabular} & \begin{tabular}{|c|} 
Treated: From \\
$0.71 \pm 0.12$ to \\
$0.48 \pm 0.34$ \\
(LogMAR) \\
FE control: \\
From $0.84 \pm$ \\
0.23 to $0.98 \pm$ \\
0.41 \\
(LogMAR) \\
(P $<0.05$ )
\end{tabular} & $\begin{array}{c}\text { Treated: From } \\
0.35 \pm 0.23 \text { to } \\
0.24 \pm 0.77 \\
\text { (LogMAR) } \\
\text { FE control: } \\
\text { From } 0.46 \pm \\
0.21 \text { to } 0.64 \pm \\
0.39 \text { (LogMAR) } \\
(\mathrm{P}<0.05)\end{array}$ & - \\
\hline $\begin{array}{c}\text { Leccisotti et al, } \\
2010[47]\end{array}$ & $\begin{array}{c}\text { Prospective, } \\
\text { consecutive, single- } \\
\text { masked, paired-eye } \\
\text { study } / 3 \mathrm{~mW} / \mathrm{cm}^{2} 30 \\
\mathrm{~min}\end{array}$ & $\begin{array}{l}102 ; 51 \\
\text { treated, } \\
51 \mathrm{FE} \\
\text { control }\end{array}$ & 12 & $\begin{array}{c}\text { Myopia/ } \\
\text { astigmatism } \uparrow 1 \mathrm{D} \text { or } \\
\text { average SIM K } \uparrow \\
1.50 \mathrm{D} / 12 \text { months }\end{array}$ & $\begin{array}{c}\text { CBM Vega X-linker/ } \\
0.1 \% \text { riboflavin with } 20 \% \\
\text { dextran T500 and } \\
\text { oxybuprocaine }\end{array}$ & $\begin{array}{c}\text { Improved mean CDVA, decreased } \\
\text { mean SE refraction, reduced } \\
\text { increase of mean apex curvature, } \\
\text { decreased mean average simulated } \\
\mathrm{K} \text {, reduced increase of mean index } \\
\text { of surface variance. }\end{array}$ & \begin{tabular}{|c|} 
Mean \\
average SIM \\
K (treated): \\
$46.63 \pm 2.89$ \\
Mean \\
average SIM \\
K (control): \\
$44.60 \pm 2.19$ \\
\end{tabular} & $\begin{array}{c}\text { Treated: } \\
-0.10 \pm 1.44 \\
\text { Control: } \\
0.88 \pm 2.35 \\
(\mathrm{P}<0.05)\end{array}$ & - & \begin{tabular}{|c|} 
Treated: -0.036 \\
\pm 0.049 \\
(LogMAR) \\
Control: +0.039 \\
\pm 0.032 \\
(LogMAR) \\
$(\mathrm{P}<0.05)$
\end{tabular} & \begin{tabular}{|c|} 
Mean SE \\
(treated): \\
$+0.35 \pm 0.66$ \\
Mean SE \\
(control): \\
$-0.83 \pm 0.88$ \\
$(\mathrm{P}<0.05)$
\end{tabular} \\
\hline $\begin{array}{l}\text { Vinciguerra } e t \\
a l, 2014[44]\end{array}$ & $\begin{array}{l}\text { Prospective non- } \\
\text { randomised clinical } \\
\text { study } / 10 \mathrm{~mW} / \mathrm{cm}^{2} 9 \\
\mathrm{~min}\end{array}$ & 20 & 12 & $\begin{array}{c}1 . \Delta \text { curvature in } \\
\text { cone area of } \geq 1 \mathrm{D} \\
2 . \text { Thinning of }> \\
20 \mu \mathrm{m} \text { in minimal } \\
\text { Scheimpflug corneal } \\
\text { thickness }\end{array}$ & $\begin{array}{c}\text { UV-X 2000; IROC/ } 0.1 \% \\
\text { riboflavin, with EDTA } \\
\text { and trometamol, dextran- } \\
\text { free or sodium chloride } \\
\text { administered by } \\
\text { iontophoresis (I-ON XL, } \\
\text { SOOFT) }\end{array}$ & $\begin{array}{c}\text { Improved CDVA. Aberrometry } \\
\text { remained stable and a trend towards } \\
\text { improvement. No progression of } \\
\text { keratoconus. }\end{array}$ & $\begin{array}{c}\text { Max K: } \\
59.07 \pm 3.90\end{array}$ & $\begin{array}{c}-0.549 \pm \\
2.344(\mathrm{P}= \\
0.40)\end{array}$ & - & $\begin{array}{c}-0.12 \pm 0.06 \\
(\operatorname{LogMAR})(\mathrm{P}= \\
0.01)\end{array}$ & \begin{tabular}{|c|} 
SE: $+1.117 \pm$ \\
$3.783(\mathrm{P}=$ \\
$0.20)$
\end{tabular} \\
\hline $\begin{array}{c}\text { Koppen et al, } \\
2012[48]\end{array}$ & $\begin{array}{c}\text { Prospective cohort } \\
\text { study } / 3 \mathrm{~mW} / \mathrm{cm}^{2} 30 \\
\mathrm{~min}\end{array}$ & 53 & 18 & $\begin{array}{l}\text { 1. } \uparrow \max K \geq 1 \mathrm{D} \\
\text { 2. } \downarrow \text { visual acuity } \\
\text { and refraction }\end{array}$ & $\begin{array}{c}\text { Vega CBM X-linker/ } \\
0.1 \% \text { riboflavin in } 20.0 \% \\
\text { dextran }\end{array}$ & $\begin{array}{l}\text { Only corrected distance visual } \\
\text { acuity showed significant } \\
\text { improvement. Maximum K and } \\
\text { pachymetry at the thinnest point } \\
\text { continued to progress. }\end{array}$ & \begin{tabular}{|c|} 
SIM K \\
steepest: \\
$48.69 \pm 5.39$
\end{tabular} & $\begin{array}{c}+0.48 \pm 0.28 \\
(\mathrm{P}>0.05)\end{array}$ & - & $\begin{array}{l}+0.05 \pm 0.03 \\
(\mathrm{SDE})(\mathrm{P}> \\
0.05)\end{array}$ & \begin{tabular}{|c|} 
Sphere: + \\
$0.04 \pm 0.21(\mathrm{P}$ \\
$>0.05)$ \\
Cyl: $-0.08 \pm$ \\
$0.19(\mathrm{P}>$ \\
$0.05)$
\end{tabular} \\
\hline
\end{tabular}




\begin{tabular}{|c|c|c|c|c|c|c|c|c|c|c|c|}
\hline Study & $\begin{array}{l}\text { Study design/ } \\
\text { Protocol }\end{array}$ & $\begin{array}{c}\text { No. of } \\
\text { Eves }\end{array}$ & $\begin{array}{c}\text { Follow-up, } \\
\text { months }\end{array}$ & $\begin{array}{l}\text { Criteria for } \\
\text { Progression }\end{array}$ & $\begin{array}{c}\text { UV device/ UV energy/ } \\
\text { Riboflavin }\end{array}$ & \multicolumn{6}{|c|}{ Outcome } \\
\hline - & - & - & - & - & - & Overall & $\begin{array}{l}\text { Pre-op K } \\
\text { (D) }\end{array}$ & $\Delta K(D)$ & $\triangle \mathrm{UCVA}$ & $\triangle \mathrm{BCVA}$ & $\begin{array}{l}\Delta \text { Refraction } \\
\text { (D) }\end{array}$ \\
\hline $\begin{array}{l}\text { Caporossi } e t \\
a l, 2013[40]\end{array}$ & $\begin{array}{c}\text { Prospective case } \\
\text { series } / 3 \mathrm{~mW} / \mathrm{cm}^{2} 30 \\
\mathrm{~min}\end{array}$ & 26 & 24 & \begin{tabular}{|c|} 
1. $\downarrow$ UDVA and/or \\
CDVA $>1$ Snellen \\
line \\
$2 . \uparrow$ sphere and/or \\
cyl $>0.50 \mathrm{D}$ \\
$3 . \uparrow$ topographic \\
symmetry index \\
surface asymmetry \\
index and/or \\
symmetry index $>$ \\
$0.50 \mathrm{D}$ \\
$4 . \uparrow$ max $\mathrm{K}>1 \mathrm{D}$ \\
$5 . \downarrow$ thinnest point \\
on AC OCT $\geq 10 \mu \mathrm{m}$
\end{tabular} & $\begin{array}{l}\text { CBM X-linker, VEGA/ } \\
5.4 \mathrm{~J} / \mathrm{cm}^{2} / 0.1 \% \text { riboflavin } \\
\text { with } 15.0 \% \text { dextran, } \\
\text { trometamol and EDTA }\end{array}$ & $\begin{array}{l}\text { UDVA and CDVA improved in the } \\
\text { first } 3-6 \text { months but returned to } \\
\text { baseline. Simulated maximum K } \\
\text { value worsened at } 24 \text { months. } \\
\text { Spherical aberration increased at } 24 \\
\text { months. }\end{array}$ & $\begin{array}{l}\text { Max K: } \\
48.59\end{array}$ & $\begin{array}{c}+1.55(\mathrm{P}= \\
0.05)\end{array}$ & $\begin{array}{c}-0.05 \text { Snellen } \\
\text { lines }(\mathrm{P}= \\
0.61)\end{array}$ & $\begin{array}{c}+0.05 \text { Snellen } \\
\text { lines }(\mathrm{P}=0.57)\end{array}$ & - \\
\hline $\begin{array}{l}\text { Bikbova et al, } \\
2014[43]\end{array}$ & $\begin{array}{c}\text { Prospective case } \\
\text { series } / 3 \mathrm{~mW} / \mathrm{cm}^{2} 30 \\
\mathrm{~min}\end{array}$ & 22 & 12 & $\begin{array}{c}1 . \uparrow \text { steepest } \mathrm{K} \text { by } \geq \\
1 \mathrm{D} \text { in manifest cyl } \\
2 . \uparrow \geq 0.5 \mathrm{D} \text { in } \\
\text { manifest SE }\end{array}$ & $\begin{array}{l}\text { UFalink/ Riboflavin } 0.1 \% \\
\text { solution administered by } \\
\text { iontophoresis (Potok-1) }\end{array}$ & $\begin{array}{l}\text { Decreased average } \mathrm{K} \text { level, corneal } \\
\text { astigmatism. Improved UDVA. }\end{array}$ & $\begin{array}{c}\text { Max K: } \\
47.82 \pm 2.23\end{array}$ & $\mid \begin{array}{c}\text { From } 47.82 \\
\pm 2.23 \text { to } \\
45.72 \pm 2.13\end{array}$ & $\begin{array}{c}\text { From } 0.61 \pm \\
0.44 \text { to } 0.48 \pm \\
0.41\end{array}$ & \begin{tabular}{|c|} 
From $0.34 \pm$ \\
0.29 to $0.29 \pm$ \\
$0.25($ LogMAR $)$ \\
$(\mathrm{P}>0.062)$
\end{tabular} & $\begin{array}{l}\text { Cyl: From } \\
3.44 \pm 0.48 \text { to } \\
2.95 \pm 0.23\end{array}$ \\
\hline
\end{tabular}

$U V=$ Ultraviolet $p r e-o p=$ pre-operative $F E=$ Fellow-Eye $U C V A=$ Uncorrected Visual Acuity $B C V A=$ Best Corrected Visual Acuity

$U D V A=$ Uncorrected Distance Visual Acuity $C D V A=$ Corrected Distance Visual Acuity $C V A=$ Corrected Visual Acuity Kmax = maximum keratometry


$H O A=$ Higher-Order Abberations

$A C O C T=$ Anterior Chamber Optical Coherence Tomography $D=$ Diopters $c y l=$ cylinder $\max =\operatorname{maximum} S I M=$ simulated 
Table 3B. Summary of outcomes for epithelium-on (transepithelial) cross-linking (Pediatrics).

\begin{tabular}{|c|c|c|c|c|c|c|c|c|c|c|c|}
\hline \multirow[t]{2}{*}{ Study } & \multirow{2}{*}{$\begin{array}{c}\text { Study design/ } \\
\text { Protocol }\end{array}$} & \multirow{2}{*}{$\begin{array}{c}\text { No. of } \\
\text { eyes }\end{array}$} & \multirow{2}{*}{$\begin{array}{c}\begin{array}{c}\text { Follow-up, } \\
\text { months }\end{array} \\
\end{array}$} & \multirow{2}{*}{$\begin{array}{l}\text { Criteria for } \\
\text { Progression }\end{array}$} & \multirow{2}{*}{$\begin{array}{c}\text { UV device/ UV energy/ } \\
\text { Riboflavin }\end{array}$} & \multicolumn{6}{|c|}{ Outcome } \\
\hline & & & & & & Overall & $\begin{array}{l}\text { Pre-op K } \\
\text { (D) }\end{array}$ & $\Delta \mathbf{K}(\mathbf{D})$ & $\triangle \mathrm{UCVA}$ & $\triangle B C V A$ & \begin{tabular}{|c|}
$\Delta$ Refraction \\
(D)
\end{tabular} \\
\hline $\begin{array}{c}\text { Salman, } 2013 \\
{[38]}\end{array}$ & $\begin{array}{c}\text { Prospective } \\
\text { comparative case } \\
\text { series } / 3 \mathrm{~mW} / \mathrm{cm}^{2} 30 \\
\mathrm{~min}\end{array}$ & $\begin{array}{l}44 ; 22 \\
\text { epi-on, } \\
22 \mathrm{FE} \\
\text { control }\end{array}$ & 12 & \begin{tabular}{|c|}
$1 . \mathrm{K}>45.0 \mathrm{D}$ \\
2. Inferior \\
steepening $>1.0 \mathrm{D}$ \\
in superior half of \\
cornea \\
$3.1 .0 \mathrm{D}$ of \\
tomographic cyl \\
progression $/ 1$ year \\
$4 . \downarrow \mathrm{CDVA}$ \\
$5 . \mathrm{New} \mathrm{CL}$ \\
fitting $/ 2$ years \\
\end{tabular} & $\begin{array}{c}\text { Opto XLink/ } 5.4 \mathrm{~J} / \mathrm{cm}^{2} / \\
0.1 \% \text { riboflavin with } 15.0 \% \\
\text { dextran, trometamol and } \\
\text { EDTA }\end{array}$ & $\begin{array}{l}\text { Improved mean UDVA, } \\
\text { decreased mean simulated } \mathrm{K} \text {, } \\
\text { mean flattening of apical } \mathrm{K}\end{array}$ & $\begin{array}{c}\text { Mean SIM } \\
\text { K (treated): } \\
49.98 \pm 4.46 \\
\text { Mean SIM } \\
\text { K (FE } \\
\text { control): } \\
48.78 \pm 3.46\end{array}$ & $\begin{array}{c}\text { Mean SIM K } \\
\text { (treated): }-2.03 \\
(\mathrm{P}<0.05) \\
\text { Mean SIM K } \\
(\text { FE control): } \\
+0.59(\mathrm{P}> \\
0.05)\end{array}$ & \begin{tabular}{|c} 
Treated: From \\
$0.95 \pm 0.34$ to \\
$0.68 \pm 0.45$ \\
(LogMAR) (P $<$ \\
$0.023)$ \\
FE control: \\
From $0.84 \pm$ \\
0.52 to $0.94 \pm$ \\
0.22 (LogMAR) \\
(P = 0.324)
\end{tabular} & \begin{tabular}{|c} 
Treated: From 0.51 \\
\pm 0.11 to $0.49 \pm$ \\
0.09 (LogMAR) $(\mathrm{P}$ \\
$=0.189)$ \\
FE control: From \\
$0.42 \pm 0.11$ to 0.51 \\
\pm 0.21 (LogMAR) \\
$(\mathrm{P}=0.543)$
\end{tabular} & \begin{tabular}{|c} 
SE (treated): - \\
From $3.17 \pm$ \\
2.72 to $-2.87 \pm$ \\
$2.86(\mathrm{P}=$ \\
$0.751)$ \\
$\mathrm{SE}$ (control): \\
$-3.72 \pm 4.72$ to \\
$-4.12 \pm 2.42$ (P \\
$=0.032)$
\end{tabular} \\
\hline $\begin{array}{l}\text { Buzzonetti et } \\
\text { al, } 2012[36]\end{array}$ & $\begin{array}{c}\text { Prospective case } \\
\text { series } / 3 \mathrm{~mW} / \mathrm{cm}^{2} 30 \\
\mathrm{~min}\end{array}$ & 13 & 18 & - & $\begin{array}{c}\text { CBM X-linker, VEGA/ } \\
0.1 \% \text { riboflavin with } 15.0 \% \\
\text { dextran, trometamol and } \\
\text { EDTA }\end{array}$ & $\begin{array}{l}\text { Improved CDVA but } \mathrm{K} \\
\text { readings and HOAs showed } \\
\text { significant worsening }\end{array}$ & $\begin{array}{c}\text { Kmax: } 48.90 \\
\pm 3.60\end{array}$ & $\begin{array}{c}\text { From } 48.90 \pm \\
3.60 \text { to } 52.90 \pm \\
4.90(\mathrm{P}<0.05)\end{array}$ & - & \begin{tabular}{|c|} 
From $0.19 \pm 0.14$ to \\
$0.1 \pm 0.1$ \\
(LogMAR) $(\mathrm{P}<$ \\
$0.05)$
\end{tabular} & $\begin{array}{l}\text { SE: From }-3.10 \\
\pm 2.40 \text { to }-3.50 \\
\quad \pm 2.90\end{array}$ \\
\hline $\begin{array}{l}\text { Buzzonetti et } \\
a l, 2015[45]\end{array}$ & $\begin{array}{c}\text { Prospective case } \\
\text { series } / 10 \mathrm{~mW} / \mathrm{cm}^{2} 9 \\
\mathrm{~min}\end{array}$ & 14 & 15 & - & $\begin{array}{c}\text {-/Riboflavin solution } \\
\text { administered by } \\
\text { iontophoresis (I-ON CXL) }\end{array}$ & $\begin{array}{c}\text { CDVA improved from } 0.7 \pm \\
1.7 \text { to } 0.8 \pm 1.8 . \text { Unchanged } \\
\text { SE, refractive astigmatism, } \\
\text { topographic and } \\
\text { aberrometric data. } \\
\text { Unchanged mean thinnest } \\
\text { point and endothelial cell } \\
\text { density. }\end{array}$ & $\begin{array}{c}\text { Kmax: } 47.6 \\
\quad \pm 2.0\end{array}$ & \begin{tabular}{|c} 
From $47.6 \pm$ \\
2.0 to $48.0 \pm$ \\
$2.3(\mathrm{P}=0.08)$
\end{tabular} & - & $\begin{array}{c}\text { From } 0.7 \pm 1.7 \text { to } \\
0.8 \pm 1.8 \\
\text { (LogMAR) }(\mathrm{P}= \\
0.005)\end{array}$ & $\begin{array}{c}\text { From }-2.2 \pm 2.7 \\
\text { to }-1.5 \pm 1.8(\mathrm{P} \\
=0.3)\end{array}$ \\
\hline $\begin{array}{l}\text { Magli et al, } \\
2016[46]\end{array}$ & $\begin{array}{c}\text { Prospective case } \\
\text { series } / 10 \mathrm{~mW} / \mathrm{cm}^{2} 9 \\
\mathrm{~min}\end{array}$ & 13 & 18 & $\begin{array}{c}\uparrow \text { max cone apex } \\
\text { curvature } \geq 1 \mathrm{D} / 6 \\
\text { months }\end{array}$ & $\begin{array}{c}\text { UV-X 2000; IROC/ } \\
\text { Riboflavin 0.1\% with } \\
\text { EDTA and tromethamine } \\
\text { without dextran or sodium } \\
\text { chloride administered by } \\
\text { iontophoresis (I-ON XL, } \\
\text { SOOFT) }\end{array}$ & $\begin{array}{c}\text { Stabilisation of refractive } \\
\text { UCVA and BCVA as early } \\
\text { as } 1 \text { month after CXL. Kmax } \\
\text { remained stable. Pediatric } \\
\text { keratoconus progression } \\
\text { halted. }\end{array}$ & $\begin{array}{c}\text { Kmax: } 53.26 \\
\pm 3.88\end{array}$ & \begin{tabular}{|c|} 
From $53.26 \pm$ \\
3.88 to $53.98 \pm$ \\
$7.94(\mathrm{P}=0.04)$
\end{tabular} & $\begin{array}{c}\text { From } 0.67 \pm \\
0.22 \text { to } 0.63 \pm \\
0.36(\text { LogMAR }) \\
(P=0.05)\end{array}$ & $\begin{array}{c}\text { From } 0.45 \pm 0.28 \text { to } \\
0.42 \pm 0.22 \\
(\operatorname{LogMAR})(\mathrm{P}= \\
0.03)\end{array}$ & - \\
\hline
\end{tabular}




\begin{tabular}{|c|c|c|c|c|c|c|c|c|c|c|c|}
\hline \multirow[t]{2}{*}{ Study } & \multirow{2}{*}{$\begin{array}{c}\text { Study design/ } \\
\text { Protocol }\end{array}$} & \multirow{2}{*}{$\begin{array}{c}\text { No. of } \\
\text { eyes }\end{array}$} & \multirow{2}{*}{\begin{tabular}{|c|}
$\begin{array}{c}\text { Follow-up, } \\
\text { months }\end{array}$ \\
\end{tabular}} & \multirow{2}{*}{$\begin{array}{l}\text { Criteria for } \\
\text { Progression }\end{array}$} & \multirow{2}{*}{$\begin{array}{c}\text { UV device/ UV energy/ } \\
\text { Riboflavin }\end{array}$} & \multicolumn{6}{|c|}{ Outcome } \\
\hline & & & & & & Overall & $\begin{array}{l}\text { Pre-op K } \\
\text { (D) }\end{array}$ & $\Delta K(D)$ & $\triangle \mathrm{UCVA}$ & $\triangle B C V A$ & $\begin{array}{l}\Delta \text { Refraction } \\
\text { (D) }\end{array}$ \\
\hline $\begin{array}{l}\text { Magli et al, } \\
2013 \text { [39] }\end{array}$ & $\begin{array}{l}\text { Retrospective/ } \\
3 \mathrm{~mW} / \mathrm{cm}^{2} 30 \mathrm{~min}\end{array}$ & $\begin{array}{l}37 ; 14 \\
\text { epi-on, } \\
23 \text { epi- } \\
\text { off }\end{array}$ & 12 & $\begin{array}{c}\uparrow \max \text { cone apex } \\
\text { curvature } \geq 1 \mathrm{D} / 6 \\
\text { months }\end{array}$ & $\begin{array}{c}\text { Epi-on: Vega/ } 0.1 \% \\
\text { riboflavin with } 15.0 \% \\
\text { dextran, trometamol and } \\
\text { EDTA } \\
\text { Epi-off: Vega CBM X } \\
\text { linker/ } 0.1 \% \text { riboflavin in } \\
20 \% \text { dextran }\end{array}$ & $\begin{array}{l}\text { Significant reduction in } \\
\text { Kmax, Kmin, mean K in } \\
\text { both the epi-off and epi-on } \\
\text { groups. }\end{array}$ & \begin{tabular}{|c} 
Epi-off: \\
$50.13 \pm 4.0$ \\
Epi-on: \\
$49.27 \pm 4.1$
\end{tabular} & $\begin{array}{c}\text { Kmax (Epi- } \\
\text { off): }-1.11(\mathrm{P}= \\
0.01) \\
\text { Kmax (Epi-on): } \\
-1.14(\mathrm{P}=0.02)\end{array}$ & \begin{tabular}{|c} 
Epi-off: From \\
$0.68 \pm 0.21$ to \\
$0.67 \pm 0.24$ \\
(LogMAR) $(\mathrm{P}=$ \\
$0.1)$ \\
Epi-on: From \\
$0.55 \pm 0.33$ to \\
$0.54 \pm 0.22$ \\
(LogMAR) (P $=$ \\
$0.3)$
\end{tabular} & $\begin{array}{c}\text { Epi-off: From } 0.36 \\
\pm 0.1 \text { to } 0.36 \pm 0.1 \\
\text { (LogMAR) }(\mathrm{P}= \\
0.8) \\
\text { Epi-on: From } 0.26 \\
\pm 0.2 \text { to } 0.27 \pm 0.2 \\
\text { (LogMAR) }(\mathrm{P}= \\
0.5)\end{array}$ & - \\
\hline
\end{tabular}

$U V=$ Ultraviolet $p r e-o p=$ pre-operative $F E=$ Fellow-Eye $U C V A=$ Uncorrected Visual Acuity $B C V A=$ Best Corrected Visual Acuity

$U D V A=$ Uncorrected Distance Visual Acuity $C D V A=$ Corrected Distance Visual acuity Kmax $=$ maximum keratometry Kmin = minimum keratometry

$K=$ keratometry epi-on = epithelium-on epi-off = epithelium-off $E D T A=$ sodium Ethylenediaminetetraacetic Acid

Trometamol $=$ Tris-hydroxymethyl aminomethane $S E=$ Spherical Equivalent $C X L=$ Cross-Linking $H O A=$ Higher-Order Abberations $C L=$ Contact Lens $D=\operatorname{diopters} c y l=$ cylinder $S I M=\operatorname{simulated} m a x=$ maximum

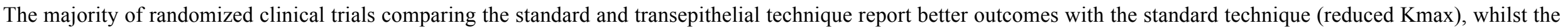
transepithelial technique had worsening of keratometry measurements (Kmax) [41, 42].

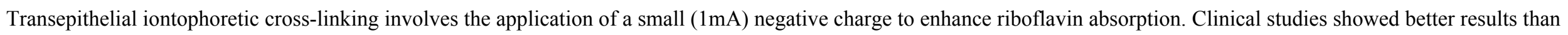


treatment of paediatric keratoconus using the accelerated protocol with favorable outcome (improved visual acuity and stability of refraction and topography) [45, 46].

\section{ACCELERATED CROSS-LINKING (TABLES 4A and 4B)}

The Bunsen-Roscoe Law of Reciprocity states that the photochemical biological effect of ultraviolet light is proportional to the total energy dose delivered, regardless of the

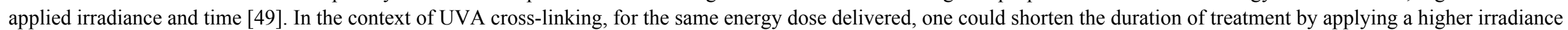

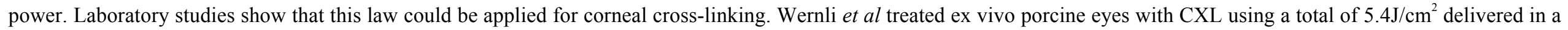
range of irradiances from 3 to $90 \mathrm{~mW} / \mathrm{cm}^{2}$. Significant stiffening was observed in eyes treated with irradiances from 3 to $45 \mathrm{~mW} / \mathrm{cm}^{2}[50]$. 
Table 4A. Summary of outcomes for accelerated cross-linking (comparative studies).

\begin{tabular}{|c|c|c|c|c|c|c|c|c|c|c|c|c|}
\hline Study & $\begin{array}{l}\text { Study design/ } \\
\text { Indication// }\end{array}$ & No. of Eyes & $\begin{array}{c}\text { Follow-up, } \\
\text { months }\end{array}$ & $\begin{array}{l}\text { Criteria for } \\
\text { Progression }\end{array}$ & $\begin{array}{l}\text { UV device/ } \\
\text { Riboflavin }\end{array}$ & Protocol & \multicolumn{6}{|c|}{ Outcome } \\
\hline - & - & - & - & - & - & - & Overall & Pre-op K (D) & $\Delta \mathrm{K}(\mathrm{D})$ & $\triangle$ UCVA & $\triangle$ BCVA & $\begin{array}{l}\Delta \text { Refraction } \\
\text { (D) }\end{array}$ \\
\hline $\begin{array}{c}\text { Kanellopoulos, } \\
2012[51]\end{array}$ & $\begin{array}{l}\text { Prospective, } \\
\text { randomised } \\
\text { bilateral } \\
\text { comparison trial/ } \\
\text { Keratoconus }\end{array}$ & $\begin{array}{l}\text { 42; } 21 \text { Group } \\
\text { A (treated), } 21 \\
\text { Group B (FE } \\
\text { control) }\end{array}$ & $\begin{array}{c}18-56 \\
\text { (mean 46) }\end{array}$ & \begin{tabular}{|c|}
$\mathrm{K}>45$ and $/$ or \\
inferior \\
steepening $>1 \mathrm{D}$ \\
to the superior \\
half of the \\
cornea \\
and $1 \mathrm{D}$ of \\
tomographic cyl \\
progression/ 1 \\
year
\end{tabular} & $\begin{array}{l}-/ 0.1 \% \\
\text { riboflavin } 5 \text { min }\end{array}$ & $\begin{array}{c}\text { Group A: } \\
7 \mathrm{~mW} / \mathrm{cm}^{2} 15 \\
\text { min } \\
\text { Group B: } \\
3 \mathrm{~mW} / \mathrm{cm}^{2} 30 \\
\text { min }\end{array}$ & $\begin{array}{c}\text { Improved UDVA and } \\
\text { BCVA in both } \\
\text { groups. Reduced } \\
\text { mean sphere, mean } \\
\text { cyl and steepest K. }\end{array}$ & - & $\begin{array}{c}\text { Group A: } 49.5 \\
\text { to } 46.1 \\
\text { Group B: From } \\
48.7 \text { to } 45.8\end{array}$ & $\begin{array}{c}\text { Group A: } \\
\text { From 20/60 to } \\
20 / 38 \\
\text { Group B: } \\
\text { From 20/62 to } \\
20 / 40\end{array}$ & $\begin{array}{c}\text { Both groups: } \\
\text { From 20/30 to } \\
20 / 25\end{array}$ & $\begin{array}{c}\text { SE (Group A): } \\
-2.5 \\
\text { SE (Group B): } \\
-2.3\end{array}$ \\
\hline $\begin{array}{l}\text { Shetty et al, } \\
2015 \text { [56] }\end{array}$ & $\begin{array}{c}\text { Prospective } \\
\text { randomised } \\
\text { interventional } \\
\text { study/ } \\
\text { Keratoconus }\end{array}$ & $\begin{array}{c}138 ; \\
36 \text { Group 1, } \\
36 \text { Group 2, } \\
33 \text { Group 3, } \\
33 \text { Group } 4\end{array}$ & 12 & $\mid \begin{array}{c}\uparrow \text { steep } \mathrm{K} \text { by }> \\
1.0-1.5 \mathrm{D}, \mathrm{a} \\
\text { corresponding } \Delta \\
(>1.0-1.5 \mathrm{D}) \text { in } \\
\text { subjective } \\
\text { refraction or a } \downarrow \\
\geq 5 \% \text { in thinnest } \\
\text { pachymetry/ } 6 \\
\text { months }\end{array}$ & $\begin{array}{c}\text { Avedro KXL/ } \\
0.1 \% \text { riboflavin } \\
\text { with } 20 \% \\
\text { dextran } 30 \mathrm{~min}\end{array}$ & $\begin{array}{c}\text { Group 1: } \\
3 \mathrm{~mW} / \mathrm{cm}^{2} 30 \\
\text { min } \\
\text { Group 2: } \\
9 \mathrm{~mW} / \mathrm{cm}^{2} 10 \\
\text { min } \\
\text { Group 3: } \\
18 \mathrm{~mW} / \mathrm{cm}^{2} 5 \\
\text { min } \\
\text { Group 4: } \\
30 \mathrm{~mW} / \mathrm{cm}^{2} 3 \\
\mathrm{~min}\end{array}$ & \begin{tabular}{|c} 
Improved mean \\
CDVA and SE in all \\
groups except Group \\
4, with Group 3 \\
showing the best \\
results. Flattening of \\
steep and flat K was \\
significant in Groups \\
1 and 2 . Groups 1 and \\
2 showed a good \\
demarcation line.
\end{tabular} & \begin{tabular}{|c} 
Steep K (Group \\
1): $50.5 \pm 4.2$ \\
Steep K (Group \\
2): $49.9 \pm 3.8$ \\
Steep K (Group \\
3): $48.6 \pm 3.5$ \\
Steep K (Group \\
4): $49.4 \pm 4.2$ \\
$(\mathrm{P}=0.23)$
\end{tabular} & \begin{tabular}{|c|} 
Group 1: 1.32 \\
$(\mathrm{P}<0.001)$ \\
Group 2: 0.67 \\
$(\mathrm{P}<0.006)$ \\
Group 3: 0.52 \\
$\quad(\mathrm{P}<0.03)$ \\
Group 4: -0.18
\end{tabular} & - & $\begin{array}{c}\text { Group 1: } 0.04 \\
\text { (SDE) }(\mathrm{P}< \\
0.05) \\
\text { Group 2: } 0.06 \\
\text { (SDE) }(\mathrm{P}< \\
0.05) \\
\text { Group 3: } 0.10 \\
\text { (SDE) }(\mathrm{P}< \\
0.05) \\
\text { Group 4: } 0.02 \\
\text { (SDE) (P }< \\
0.05)\end{array}$ & $\begin{array}{l}\text { Group 1: - } 0.85 \\
\quad(\mathrm{P}<0.01) \\
\text { Group 2: }-1(\mathrm{P} \\
\quad<0.01) \\
\text { Group 3: - } 1.68 \\
\quad(\mathrm{P}<0.01) \\
\text { Group 4: - } 0.49 \\
\quad(\mathrm{P}=0.12)\end{array}$ \\
\hline $\begin{array}{c}\text { Sherif, } 2014 \\
\text { [58] }\end{array}$ & $\begin{array}{c}\text { Prospective } \\
\text { randomised } \\
\text { interventional } \\
\text { case-control } \\
\text { clinical trial/ } \\
\text { Keratoconus }\end{array}$ & \begin{tabular}{|c}
$25 ; 14$ \\
accelerated, \\
11 \\
conventional
\end{tabular} & 12 & $\begin{array}{c}\uparrow \geq 1.0 \mathrm{D} \text { in } \\
\text { steepest } \mathrm{K}, \uparrow \geq \\
1.0 \mathrm{D} \text { in manifest } \\
\text { cyl, or } \uparrow \geq 0.5 \mathrm{D} \\
\text { in MRSE/ } 6 \\
\text { months }\end{array}$ & $\begin{array}{c}0.1 \% \text { riboflavin } \\
\text { with dextran } 30 \\
\text { min }\end{array}$ & $\begin{array}{c}\text { Accelerated: } \\
30 \mathrm{~mW} / \mathrm{cm}^{2} \\
4 \mathrm{~min} 20 \mathrm{~s} \\
\text { Conventional: } \\
3 \mathrm{~mW} / \mathrm{cm}^{2} 30 \\
\mathrm{~min}\end{array}$ & $\begin{array}{l}\text { Decreased flat K, } \\
\text { steep K and mean K } \\
\text { in both groups. } \\
\text { Improved BSCVA. }\end{array}$ & $\begin{array}{c}\text { Max K } \\
\text { (accelerated): } \\
49.43 \pm 1.63 \\
\text { Max K } \\
\text { (conventional): } \\
51.4 \pm 1.69\end{array}$ & \begin{tabular}{|c|} 
Accelerated: \\
From $49.43 \pm$ \\
1.63 to $48.2 \pm$ \\
$1.43(\mathrm{P}=0.022)$ \\
Conventional: \\
From $51.4 \pm$ \\
1.69 to $50.24 \pm$ \\
$2(\mathrm{P}=0.099)$
\end{tabular} & - & $\begin{array}{l}\text { Accelerated: } \\
\text { From } 0.48 \pm \\
0.17 \text { to } 0.61 \pm \\
0.15 \text { (SDE) } \\
\text { (P=0.015) } \\
\text { Conventional: } \\
\text { From } 0.49 \pm \\
0.19 \text { to } 0.64 \pm \\
0.16(\mathrm{SDE})(\mathrm{P} \\
=0.03)\end{array}$ & - \\
\hline
\end{tabular}




\begin{tabular}{|c|c|c|c|c|c|c|c|c|c|c|c|c|}
\hline \multirow{2}{*}{$\begin{array}{c}\text { Study } \\
-\end{array}$} & \multirow{2}{*}{$\begin{array}{c}\begin{array}{c}\text { Study design// } \\
\text { Indication/ } \\
\text { Protocol }\end{array} \\
-\end{array}$} & \multirow{2}{*}{$\begin{array}{c}\text { No. of Eyes } \\
-\end{array}$} & \multirow{2}{*}{\begin{tabular}{|c|}
$\begin{array}{c}\text { Follow-up, } \\
\text { months }\end{array}$ \\
- \\
\end{tabular}} & \multirow{2}{*}{$\begin{array}{c}\text { Criteria for } \\
\text { Progression }\end{array}$} & \multirow{2}{*}{$\begin{array}{c}\text { UV device/ } \\
\text { Riboflavin }\end{array}$} & \multirow{2}{*}{$\begin{array}{c}\text { Protocol } \\
-\end{array}$} & \multicolumn{6}{|c|}{ Outcome } \\
\hline & & & & & & & Overall & Pre-op K (D) & $\Delta \mathbf{K}(\mathbf{D})$ & $\triangle$ UCVA & $\triangle$ BCVA & $\begin{array}{l}\Delta \text { Refraction } \\
\text { (D) }\end{array}$ \\
\hline $\begin{array}{c}\mathrm{Ng} \text { et al, } 2016 \\
{[59]}\end{array}$ & $\begin{array}{c}\text { Comparative } \\
\text { interventional } \\
\text { study/ } \\
\text { Keratoconus }\end{array}$ & $\begin{array}{c}26 ; 12 \\
\text { accelerated, } \\
14 \\
\text { conventional }\end{array}$ & 14 & $\begin{array}{c}\uparrow>1 \mathrm{D} \text { in Kmax } \\
\uparrow>1 \mathrm{D} \text { in } \\
\text { manifest cyl } \\
\text { or } \uparrow>0.5 \mathrm{D} \text { in SE } \\
\text { over 6-12 } \\
\text { months }\end{array}$ & \begin{tabular}{|} 
Conventional: \\
UV-X 1000, \\
IROC/ \\
Accelerated: \\
UV-X 2000, \\
IROC \\
For both: \\
Isotonic $0.1 \%$ \\
riboflavin with \\
$20 \%$ dextran \\
solution 25 min
\end{tabular} & $\begin{array}{c}\text { Accelerated: } \\
9 \mathrm{~mW} / \mathrm{cm}^{2} 10 \\
\text { min } \\
\text { Conventional: } \\
3 \mathrm{~mW} / \mathrm{cm}^{2} 30 \\
\mathrm{~min}\end{array}$ & $\begin{array}{l}\text { Conventional: } \\
\text { improved CDVA, } \\
\text { reduced Kmax, } \\
\text { Kmean. } \\
\text { Accelerated: } \\
\text { unchanged CDVA, } \\
\text { Kmax, Kmean }\end{array}$ & $\begin{array}{c}\text { Kmax } \\
\text { (conventional): } \\
53.5 \pm 6.3 \\
\text { Kmax } \\
\text { (accelerated): } \\
51.6 \pm 4.0 \\
(\mathrm{P}=0.820)\end{array}$ & $\begin{array}{c}\text { Conventional: } \\
-1.8 \pm 1.8 \\
\text { Accelerated: } \\
-0.3 \pm 0.9 \\
(\mathrm{P}=0.015)\end{array}$ & - & $\begin{array}{c}\text { Conventional: } \\
-0.126 \pm 0.194 \\
\text { (LogMAR) } \\
\text { Accelerated: } \\
0.021 \pm 0.092 \\
\text { (LogMAR) } \\
(\mathrm{P}=0.060)\end{array}$ & \begin{tabular}{|c}
$\mathrm{SE}$ \\
(conventional): \\
$0.23 \pm 0.87$ \\
$\mathrm{SE}$ \\
(accelerated): \\
$0.98 \pm 3.81$ \\
$(\mathrm{P}=0.796)$
\end{tabular} \\
\hline $\begin{array}{c}\text { Chow et al, } \\
2015 \text { [60] }\end{array}$ & $\begin{array}{l}\text { Prospective, } \\
\text { interventional } \\
\text { clinical study/ } \\
\text { Keratoconus }\end{array}$ & $\begin{array}{c}38 ; 19 \\
\text { accelerated, } \\
19 \\
\text { conventional }\end{array}$ & 12 & \begin{tabular}{|c|}
$\downarrow \geq 2$ lines of \\
BCVA $+\geq 1$ of \\
the following/ 12 \\
months: \\
$1 . \uparrow \geq 1 \mathrm{D}$ in \\
steepest $\mathrm{K}$ \\
$2 . \uparrow \geq 1 \mathrm{D}$ in \\
astigmatism
\end{tabular} & \begin{tabular}{|c|} 
Conventional: \\
UV-X, IROC \\
Accelerated: \\
CCL-Vario, \\
Peschke Trade \\
GmbH \\
For both: $0.1 \%$ \\
riboflavin with \\
$20 \%$ dextran \\
solution 30 min
\end{tabular} & $\begin{array}{c}\text { Accelerated: } \\
18 \mathrm{~mW} / \mathrm{cm}^{2} 5 \\
\min \\
\text { Conventional: } \\
3 \mathrm{~mW} / \mathrm{cm}^{2} 30 \\
\mathrm{~min}\end{array}$ & $\begin{array}{l}\text { Improved UCVA and } \\
\text { BCVA, reduction in } \\
\text { SE in both groups. A } \\
\text { more effective } \\
\text { topographic flattening } \\
\text { was observed in } \\
\text { conventional CXL. }\end{array}$ & $\begin{array}{c}\text { Max K } \\
\text { (conventional): } \\
54.93 \pm 1.72 \\
\text { Max K } \\
\text { (accelerated): } \\
51.96 \pm 1.80 \\
(\mathrm{P}=0.235)\end{array}$ & $\begin{array}{c}\text { Conventional: } \\
-1.6 \pm 0.72 \\
\text { Accelerated: } \\
-0.47 \pm 0.83 \\
(\mathrm{P}=0.343)\end{array}$ & $\begin{array}{c}\text { Conventional: } \\
-0.28 \pm 0.08 \\
\text { (LogMAR) } \\
\text { Accelerated: } \\
-0.20 \pm 0.06 \\
\text { (LogMAR) } \\
(\mathrm{P}=0.508)\end{array}$ & $\begin{array}{c}\text { Conventional: } \\
0.00 \pm 0.04 \\
\text { (LogMAR) } \\
\text { Accelerated: } \\
-0.14 \pm 0.02 \\
\text { (LogMAR) (P } \\
=0.430)\end{array}$ & \begin{tabular}{|c}
$\mathrm{SE}$ \\
(conventional): \\
$-1.3 \pm 0.53$ \\
$\mathrm{SE}$ \\
(accelerated): \\
$-0.57 \pm 0.26$ \\
$(\mathrm{P}=0.554)$
\end{tabular} \\
\hline $\begin{array}{c}\text { Hashemian et } \\
a l, 2014 \text { [61] }\end{array}$ & $\begin{array}{l}\text { Prospective } \\
\text { clinical trial/ } \\
\text { Keratoconus }\end{array}$ & $\begin{array}{c}153 ; 77 \\
\text { accelerated, } \\
76 \\
\text { conventional }\end{array}$ & 15 & $\begin{array}{c}\Delta \text { Mean central } \\
\mathrm{K} \geq 1.5 \mathrm{D} \text { and } \downarrow \\
>5 \% \text { in mean } \\
\mathrm{CCT} \text { through } 3 \\
\text { consecutive } \\
\text { readings } / 6 \\
\text { months }\end{array}$ & $\begin{array}{l}\text { CCL-VARIO, } \\
\text { Peschke } \\
\text { Meditrade } \\
\text { GmbH/ } 0.1 \% \\
\text { riboflavin with } \\
20 \% \text { dextran } \\
\text { solution } 30 \text { min }\end{array}$ & $\begin{array}{c}\text { Accelerated: } \\
30 \mathrm{~mW} / \mathrm{cm}^{2} 3 \\
\text { min } \\
\text { Conventional: } \\
3 \mathrm{~mW} / \mathrm{cm}^{2} 30 \\
\mathrm{~min}\end{array}$ & $\begin{array}{c}\text { Cyl and spherical } \\
\text { components of } \\
\text { refraction improved } \\
\text { significantly. No } \\
\text { difference observed } \\
\text { between the } 2 \text { groups. }\end{array}$ & - & $\begin{array}{c}\text { Kmax } \\
\text { (conventional): } \\
-1.98 \pm 0.93 \\
\text { Accelerated: } \\
-1.85 \pm 0.99 \\
(\mathrm{P}=0.36)\end{array}$ & $\begin{array}{c}\text { Conventional: } \\
0.21 \pm 0.19 \\
\text { (LogMAR) } \\
\text { Accelerated: } \\
0.19 \pm 0.20 \\
\text { (LogMAR) } \\
(\mathrm{P}=0.64)\end{array}$ & $\begin{array}{c}\text { Conventional: } \\
0.17 \pm 0.10 \\
\text { (LogMAR) } \\
\text { Accelerated: } \\
0.16 \pm 0.09 \\
\text { (LogMAR) } \\
(\mathrm{P}=0.58)\end{array}$ & \begin{tabular}{|c} 
Sphere \\
(conventional): \\
From $-4.3 \pm 1.6$ \\
to $-2.9 \pm 2.0$ \\
Sphere \\
(accelerated): \\
$-4.8 \pm 1.9$ to \\
$-3.5 \pm 2$
\end{tabular} \\
\hline
\end{tabular}




\begin{tabular}{|c|c|c|c|c|c|c|c|c|c|c|c|c|}
\hline Study & $\begin{array}{l}\text { Study design/ } \\
\text { Indication// }\end{array}$ & No. of Eyes & $\left|\begin{array}{c}\text { Follow-up } \\
\text { months }\end{array}\right|$ & $\begin{array}{l}\text { Criteria for } \\
\text { Progression }\end{array}$ & $\begin{array}{l}\text { UV device/ } \\
\text { Riboflavin }\end{array}$ & Protocol & \multicolumn{6}{|c|}{ Outcome } \\
\hline- & - & - & - & - & - & - & Overall & Pre-op K (D) & $\Delta \mathrm{K}(\mathrm{D})$ & $\Delta$ UCVA & $\triangle$ BCVA & $\begin{array}{c}\Delta \text { Refraction } \\
\text { (D) }\end{array}$ \\
\hline $\begin{array}{l}\text { Tomita et al, } \\
2014[53]\end{array}$ & $\begin{array}{c}\text { Prospective } \\
\text { comparative study/ } \\
\text { Keratoconus }\end{array}$ & $\begin{array}{c}48 ; 30 \\
\text { accelerated, } \\
18 \\
\text { conventional }\end{array}$ & 12 & - & \begin{tabular}{|c|} 
Accelerated: \\
Avedro KXL/ \\
$0.1 \%$ riboflavin \\
with HPMC 15 \\
min \\
Conventional: \\
CCL-365 Vario, \\
Peschke \\
Meditrade/ $0.1 \%$ \\
riboflavin with \\
$20.0 \%$ dextran \\
T500 30 min \\
\end{tabular} & $\begin{array}{c}\text { Accelerated: } \\
30 \mathrm{~mW} / \mathrm{cm}^{2} 3 \\
\text { min } \\
\text { Conventional: } \\
3 \mathrm{~mW} / \mathrm{cm}^{2} 30 \\
\mathrm{~min}\end{array}$ & $\begin{array}{l}\text { Both accelerated and } \\
\text { conventional CXL } \\
\text { were safe and } \\
\text { effective. Similar } \\
\text { morphologic changes } \\
\text { and a pronounced } \\
\text { demarcation line were } \\
\text { apparent in eyes in } \\
\text { both groups } \\
\text { postoperatively. }\end{array}$ & $\begin{array}{c}\text { Mean Kmax } \\
\text { (accelerated): } \\
50.45 \pm 5.28 \\
\text { Mean Kmax } \\
\text { (conventional): } \\
48.82 \pm 4.56\end{array}$ & $\begin{array}{c}\text { Accelerated: } \\
-0.62 \pm 1.46 \\
\text { Conventional: } \\
-1.77 \pm 2.65 \\
(\mathrm{P}=0.21)\end{array}$ & - & - & $\begin{array}{c}\text { MRSE } \\
\text { (accelerated): } \\
0.64 \pm 1.84 \\
\text { MRSE } \\
\text { (conventional) } \\
0.39 \pm 0.88 \\
(\mathrm{P}=0.60)\end{array}$ \\
\hline $\begin{array}{c}\text { Kymionis et al, } \\
2014[54]\end{array}$ & $\begin{array}{c}\text { Prospective } \\
\text { comparative } \\
\text { interventional case } \\
\text { series/ } \\
\text { Keratoconus }\end{array}$ & $\begin{array}{c}21 ; 12 \\
\text { accelerated, } 9 \\
\text { conventional }\end{array}$ & 1 & - & \begin{tabular}{|c|} 
CCL-365, \\
Peschke \\
Meditrade/ $0.1 \%$ \\
riboflavin with \\
$20 \%$ dextran 30 \\
min
\end{tabular} & $\begin{array}{c}\text { Accelerated: } \\
9 \mathrm{~mW} / \mathrm{cm}^{2} 10 \\
\mathrm{~min} \\
\text { Conventional: } \\
3 \mathrm{~mW} / \mathrm{cm}^{2} 30 \\
\mathrm{~min}\end{array}$ & $\begin{array}{c}\text { The mean corneal } \\
\text { stroma demarcation } \\
\text { line depth was } 350.78 \\
\text { mum } \pm 49.34 \text { in the } \\
\text { conventional group } \\
\text { and } 288.46 \pm 42.37 \\
\text { mum in the } \\
\text { accelerated group. }\end{array}$ & $\begin{array}{c}\text { Mean K steep } \\
\text { (conventional): } \\
49.35 \pm 2.80 \\
\text { Mean K steep } \\
\text { (accelerated): } \\
47.58 \pm 2.83 \\
(\mathrm{P}=0.17)\end{array}$ & - & - & - & - \\
\hline $\begin{array}{c}\text { Kymionis et al, } \\
2014[55]\end{array}$ & $\begin{array}{c}\text { Prospective } \\
\text { comparative study/ } \\
\text { Keratoconus }\end{array}$ & \begin{tabular}{|c}
$52 ; 26$ \\
accelerated, \\
26 \\
conventional
\end{tabular} & 1 & - & \begin{tabular}{|c|} 
CCL-365, \\
Peschke \\
Meditrade/ $0.1 \%$ \\
riboflavin with \\
$20 \%$ dextran 30 \\
min
\end{tabular} & $\begin{array}{c}\text { Accelerated: } \\
9 \mathrm{~mW} / \mathrm{cm}^{2} 14 \\
\min \\
\text { Conventional: } \\
3 \mathrm{~mW} / \mathrm{cm}^{2} 30 \\
\mathrm{~min}\end{array}$ & $\begin{array}{c}\text { Corneal stromal } \\
\text { demarcation line } \\
\text { depth showed no } \\
\text { significant difference } \\
\text { for both groups. }\end{array}$ & $\begin{array}{c}\text { Mean steep K } \\
\text { (conventional): } \\
49.88 \pm 3.99 \\
\text { Mean steep K } \\
\text { (accelerated): } \\
49.17 \pm 2.90 \\
(\mathrm{P}=0.467)\end{array}$ & - & - & - & - \\
\hline
\end{tabular}




\begin{tabular}{|c|c|c|c|c|c|c|c|c|c|c|c|c|}
\hline Study & $\begin{array}{l}\text { Study design/ } \\
\text { Indication/ }\end{array}$ & No. of Eyes & $\begin{array}{c}\text { Follow-up, } \\
\text { months }\end{array}$ & $\begin{array}{l}\text { Criteria for } \\
\text { Progression }\end{array}$ & $\begin{array}{l}\text { UV device/ } \\
\text { Riboflavin }\end{array}$ & Protocol & \multicolumn{6}{|c|}{ Outcome } \\
\hline- & - & - & - & - & - & - & Overall & Pre-op K (D) & $\Delta K(D)$ & $\triangle$ UCVA & $\triangle$ BCVA & $\begin{array}{c}\Delta \text { Refraction } \\
\text { (D) }\end{array}$ \\
\hline $\begin{array}{c}\text { Mazzotta et al, } \\
2014[57]\end{array}$ & $\begin{array}{l}\text { Prospective, } \\
\text { comparative, } \\
\text { interventional } \\
\text { clinical study/ } \\
\text { Keratoconus }\end{array}$ & $\begin{array}{c}20 ; 10 \\
\text { accelerated } \\
\text { pulsed, } 10 \\
\text { accelerated } \\
\text { continuous }\end{array}$ & 12 & $\begin{array}{c}\downarrow \text { UCVA/ } \\
\text { BSCVA }>0.50 \\
\text { Snellen lines, } \uparrow \\
\text { sphere } / \text { cyl }> \\
0.50 \mathrm{D}, \uparrow \\
\text { topographic } \\
\text { symmetry index } \\
\mathrm{SAI} / \mathrm{SI}>1 \mathrm{D}, \uparrow \\
\text { mean } \mathrm{K}>1 \mathrm{D} \text { or } \\
\downarrow \text { thinnest point } \\
\text { at corneal OCT } \\
\text { pachymetry } \geq \\
10 \mu \mathrm{m} \\
\end{array}$ & $\begin{array}{c}\text { Avedro KXL/ } \\
0.1 \% \text { riboflavin } \\
\text { dextran-free } 10 \\
\text { min }\end{array}$ & $\begin{array}{c}\text { Pulsed: } \\
30 \mathrm{~mW} / \mathrm{cm}^{2} 8 \\
\text { min } \\
\text { Continuous: } \\
30 \mathrm{~mW} / \mathrm{cm}^{2} 4 \\
\mathrm{~min}\end{array}$ & $\begin{array}{c}\text { Better functional } \\
\text { outcomes and deeper } \\
\text { stromal penetration in } \\
\text { pulsed light } \\
\text { accelerated treatment. }\end{array}$ & - & $\begin{array}{c}\text { Apical K } \\
\text { (Continuous): } \\
-1.39 \pm 0.38(\mathrm{P} \\
=0.05) \\
\text { Apical K } \\
\text { (Pulsed): }+0.15 \\
\pm 0.8(\mathrm{P}= \\
0.077)\end{array}$ & \begin{tabular}{|c|} 
Pulsed: $+0.9 \pm$ \\
$1.1(\mathrm{SDE})(\mathrm{P}=$ \\
$0.10)$ \\
Continuous: \\
$+0.5 \pm 1.2$ \\
$(\mathrm{SDE})(\mathrm{P}=$ \\
$0.65)$
\end{tabular} & $\begin{array}{c}\text { Pulsed: }+1.8 \pm \\
1.3 \text { (SDE) }(\mathrm{P}= \\
0.55) \\
\text { Continuous: } \\
+1.6 \pm 1.0 \\
\text { (SDE) }(\mathrm{P}= \\
0.56)\end{array}$ & - \\
\hline $\begin{array}{c}\text { Woo et al, } 2017 \\
{[62]}\end{array}$ & $\begin{array}{l}\text { Prospective, non- } \\
\text { randomised } \\
\text { interventional } \\
\text { study }\end{array}$ & $\begin{array}{c}76 ; 47 \\
\text { accelerated, } \\
29 \\
\text { conventional }\end{array}$ & 12 & $\begin{array}{c}1 . \uparrow \geq 1 \mathrm{D} \text { in } \\
\text { steepest } \mathrm{K} \\
2 . \downarrow>5 \% \text { in } \\
\text { minimal corneal } \\
\text { thickness } \\
\text { 3. } \uparrow>1 \mathrm{D} \text { in cyl/ } \\
>0.50 \mathrm{D} \mathrm{SE} \\
\text { over } \geq 6 \text { months }\end{array}$ & $\begin{array}{c}\text { Conventional: } \\
\text { UV-X, Peschke } \\
\text { Meditrade/ } \\
\text { isotonic } \\
\text { riboflavin } 0.1 \% \\
\text { with dextran } \\
20 \% 30 \mathrm{~min} \\
\text { Accelerated: } \\
\text { Avedro KXL/ } \\
\text { dextran-free } \\
\text { riboflavin } 0.1 \% \\
10 \mathrm{~min}\end{array}$ & $\begin{array}{c}\text { Conventional: } \\
3 \mathrm{~mW} / \mathrm{cm}^{2} 30 \\
\mathrm{~min} \\
\text { Accelerated: } \\
30 \mathrm{~mW} / \mathrm{cm}^{2} 4 \\
\mathrm{~min}\end{array}$ & $\begin{array}{c}\text { Both groups showed } \\
\text { no significant } \\
\text { increase in K1, K2 } \\
\text { and Kmean from } \\
\text { baseline at } 12 \text { months. } \\
\text { No difference } \\
\text { between CXL and } \\
\text { KXL group for } \\
\text { postoperative corneal } \\
\text { topography and } \\
\text { central and minimal } \\
\text { pachymetry/ } 12 \\
\text { months. }\end{array}$ & $\begin{array}{c}\text { Steepest K } \\
\text { (conventional): } \\
52.29 \pm 5.40 \\
\text { Steepest K } \\
\text { (accelerated): } \\
52.15 \pm 5.30 \\
(\mathrm{P}=0.915)\end{array}$ & $\begin{array}{c}\text { Conventional: } \\
-0.13 \\
\text { Accelerated: } \\
-0.21 \\
(\mathrm{P}=0.829)\end{array}$ & $\begin{array}{c}\text { Conventional: } \\
-0.11 \\
\text { (LogMAR) (P } \\
=0.017) \\
\text { Accelerated: } \\
\text { no statistically } \\
\text { significant } \\
\text { change }\end{array}$ & $\begin{array}{c}\text { Conventional: } \\
-0.11 \\
\text { (LogMAR) (P } \\
=0.037) \\
\text { Accelerated: } \\
-0.08(\mathrm{P}= \\
0.004)\end{array}$ & \begin{tabular}{|c}
$\mathrm{SE}$ \\
(conventional): \\
From $-4.72 \pm$ \\
3.6 to $-3.82 \pm$ \\
$4.4(\mathrm{P}=0.247)$ \\
$\mathrm{SE}$ \\
(accelerated): \\
From $-4.30 \pm$ \\
3.1 to $-5.11 \pm$ \\
$4.07(\mathrm{P}=0.131)$
\end{tabular} \\
\hline
\end{tabular}

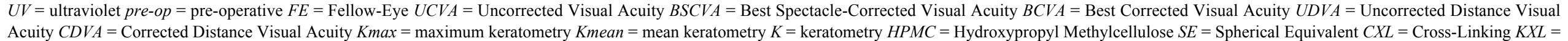
Accelerated cross-linking $C C T=$ Central Corneal Thickness

$c y l=$ cylinder $O C T=$ Optical Coherence Tomography $S A I=$ Surface Asymmetry Index $S I=$ Symmetry Index $D=$ Diopters $S D E$ : Snellen Decimal Equivalent

$M R S E=$ Manifest Refractive Spherical Equivalent $\max =$ maximum

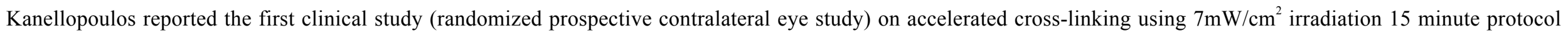



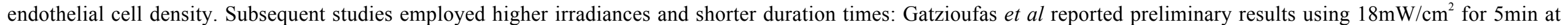



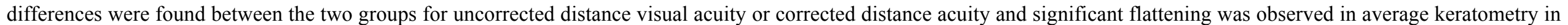






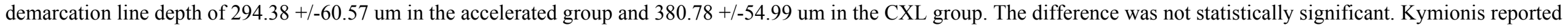

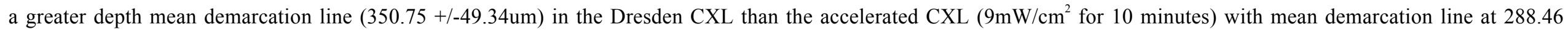

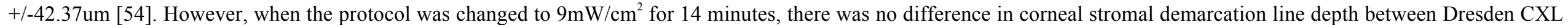
and accelerated CXL [55].

Table 4B. Summary of outcomes for accelerated cross-linking (non-comparative studies).

\begin{tabular}{|c|c|c|c|c|c|c|c|c|c|c|c|c|}
\hline \multirow[t]{2}{*}{ Study } & \multirow{2}{*}{$\begin{array}{c}\text { Study design/ } \\
\text { Indication/ } \\
\text { Protocol } \\
\end{array}$} & \multirow[t]{2}{*}{$\begin{array}{c}\text { No. } \\
\text { of } \\
\text { eyes }\end{array}$} & \multirow[t]{2}{*}{$\begin{array}{c}\text { Follow-up } \\
\text { months }\end{array}$} & \multirow[t]{2}{*}{$\begin{array}{l}\text { Criteria for } \\
\text { Progression }\end{array}$} & \multirow[t]{2}{*}{$\begin{array}{l}\text { UV device/ } \\
\text { Riboflavin }\end{array}$} & \multirow[t]{2}{*}{ Protocol } & \multicolumn{6}{|c|}{ Outcome } \\
\hline & & & & & & & Overall & Pre-op K (D) & $\Delta K(D)$ & $\triangle$ UCVA & $\triangle$ BCVA & \begin{tabular}{|c|}
$\Delta$ Refraction \\
(D)
\end{tabular} \\
\hline $\begin{array}{l}\text { Gatzioufas et } \\
a l, 2013[52]\end{array}$ & $\begin{array}{l}\text { Prospective cohort } \\
\text { study/Keratoconus }\end{array}$ & 7 & 6 & $\begin{array}{c}\text { Mean of } 3 \text { consecutive } \\
\text { measurements showing } \\
\uparrow \begin{array}{c}\text { Kmax }>1 \mathrm{D} / 12 \\
\text { months }\end{array}\end{array}$ & $\begin{array}{c}\text { CXL-365 Vario/ } \\
0.1 \% \text { riboflavin with } \\
20 \% \text { dextran } 30 \text { min }\end{array}$ & $\underset{\mathrm{min}}{18 \mathrm{~mW} / \mathrm{cm}^{2} 5}$ & $\begin{array}{c}\text { Kmax, Kmean and CDVA } \\
\text { showed no significant } \\
\text { changes after } 6 \text { months. No } \\
\text { complications were } \\
\text { observed postoperatively. }\end{array}$ & $\begin{array}{c}\text { Kmax: } 55.6 \pm \\
3.8\end{array}$ & $\begin{array}{c}\text { From } 55.6 \\
\pm 3.8 \text { to } \\
52.9 \pm 2.7 \\
(\mathrm{P}=0.42)\end{array}$ & - & \begin{tabular}{|c|} 
From $0.41 \pm$ \\
0.34 to $0.58 \pm$ \\
$0.37($ LogMAR $)$ \\
$(P=0.055)$
\end{tabular} & - \\
\hline $\begin{array}{l}\text { Shetty et al, } \\
2014 \\
\text { (paeds) [63] }\end{array}$ & $\begin{array}{c}\text { Prospective case } \\
\text { series/Keratoconus }\end{array}$ & 30 & 24 & $\begin{array}{c}\uparrow \text { steep } \mathrm{K}>1.0-1.5 \mathrm{D} \\
\text { and } \Delta \text { in subjective } \\
\text { refraction/ } 6 \text { months or } \\
\downarrow \geq 5 \% \text { in thinnest } \\
\text { pachymetry/ } 6 \text { months }\end{array}$ & $\begin{array}{c}\text { Avedro KXL/ } 0.1 \% \\
\text { riboflavin with } 20 \% \\
\text { dextran for } 30 \mathrm{~min}\end{array}$ & $\begin{array}{c}9 \mathrm{~mW} / \mathrm{cm}^{2} \text { for } \\
10 \mathrm{~min}\end{array}$ & $\begin{array}{c}\text { Improved mean UDVA, } \\
\text { mean CDVA, mean } \\
\text { spherical refraction, mean } \\
\text { cyl, and SE }\end{array}$ & $\begin{array}{c}\text { Max K: } 53.77 \pm \\
4.82\end{array}$ & \begin{tabular}{|c|} 
From \\
$53.77 \pm$ \\
4.82 to \\
$51.70 \pm$ \\
$5.41(\mathrm{P}=$ \\
$0.007)$ \\
\end{tabular} & $\begin{array}{c}\text { From } 0.76 \pm \\
0.26 \text { to } 0.61 \pm \\
0.25 \\
\text { (LogMAR) (P } \\
=0.005)\end{array}$ & $\begin{array}{c}\text { From } 0.24 \pm \\
0.19 \text { to } 0.12 \pm \\
0.12(\text { LogMAR }) \\
(\mathrm{P}<0.001)\end{array}$ & \begin{tabular}{|c|} 
Mean SE: \\
from $-4.70 \pm$ \\
3.86 to -3.75 \\
$\pm 3.49(\mathrm{P}=$ \\
$0.15)$
\end{tabular} \\
\hline $\begin{array}{l}\text { Marino et al, } \\
2015[64]\end{array}$ & $\begin{array}{c}\text { Prospective, single- } \\
\text { center case series/ } \\
\text { Post-laser ectasia }\end{array}$ & 40 & 24 & $\begin{array}{l}\text { 1. } \uparrow \text { inferior steepening } \\
2 . \uparrow \text { myopia and } \\
\text { astigmatism } \\
\text { 3. } \downarrow \text { UDVA and CDVA }\end{array}$ & \begin{tabular}{|c|} 
CCL-Vario \\
Crosslinking; \\
Peschke Meditrade \\
GmcH/ $0.1 \%$ \\
riboflavin $30 \mathrm{~min}$
\end{tabular} & $\begin{array}{c}9 \mathrm{~mW} / \mathrm{cm}^{2} \text { for } \\
10 \mathrm{~min}\end{array}$ & $\begin{array}{l}\text { All eyes stabilised after } \\
\text { treatment without any } \\
\text { further signs of } \\
\text { progression. }\end{array}$ & $\begin{array}{c}\text { Max K: } 48.89 \pm \\
2.85\end{array}$ & \begin{tabular}{|c|} 
From \\
$48.89 \pm$ \\
2.85 to \\
$49.21 \pm$ \\
$3.15(\mathrm{P}=$ \\
$0.956)$
\end{tabular} & $\begin{array}{c}\text { From } 0.33 \pm \\
0.18 \text { to } 0.37 \pm \\
0.18 \\
\text { (LogMAR) (P } \\
\quad=0.649)\end{array}$ & $\begin{array}{c}\text { From } 0.13 \pm \\
0.10 \text { to } 0.15 \pm \\
0.12(\text { LogMAR }) \\
(P=0.616)\end{array}$ & - \\
\hline $\begin{array}{l}\text { Ozgurhan et } \\
a l, 2014 \\
\text { (paeds) [65] }\end{array}$ & \begin{tabular}{|c|} 
Retrospective \\
interventional case \\
series/ Keratoconus
\end{tabular} & 44 & 24 & $\begin{array}{c}\uparrow \mathrm{Kmax} \text { of } \geq 1 \mathrm{D}, \uparrow \\
\text { astigmatism by } \geq 1 \mathrm{D} \text { or } \\
\uparrow \text { MRSE of } 0.50 \mathrm{D} / 3 \\
\text { months }\end{array}$ & $\begin{array}{c}\text { Avedro KXL/ } 0.1 \% \\
\text { riboflavin } 15 \mathrm{~min}\end{array}$ & $\begin{array}{c}30 \mathrm{~mW} / \mathrm{cm}^{2} \text { for } \\
4 \mathrm{~min}\end{array}$ & $\begin{array}{l}\text { Improved UDVA and } \\
\text { CDVA. Flat K value and } \\
\text { steep K value decreased. }\end{array}$ & \begin{tabular}{|c|} 
Max K: $50.6 \pm$ \\
4.2
\end{tabular} & \begin{tabular}{|c|} 
From 50.6 \\
\pm 4.2 to \\
$50.1 \pm 4.0$ \\
$(\mathrm{P}<$ \\
$0.001)$
\end{tabular} & $\begin{array}{c}\text { From } 0.52 \pm \\
0.36 \text { to } 0.39 \pm \\
0.26 \\
\text { (LogMAR) (P } \\
=0.002)\end{array}$ & $\begin{array}{c}\text { From } 0.38 \pm \\
0.24 \text { to } 0.30 \pm \\
0.20 \text { (LogMAR) } \\
(\mathrm{P}<0.001)\end{array}$ & \begin{tabular}{|c|} 
SE: From \\
$-5.45 \pm 2.99$ \\
to $5.27 \pm 2.91$ \\
$(\mathrm{P}=0.205)$
\end{tabular} \\
\hline
\end{tabular}




\begin{tabular}{|c|c|c|c|c|c|c|c|c|c|c|c|c|}
\hline \multirow[t]{2}{*}{ Study } & \multirow{2}{*}{$\begin{array}{l}\text { Study design/ } \\
\text { Indication// } \\
\text { Protocol } \\
\end{array}$} & \multirow{2}{*}{$\begin{array}{c}\text { No. } \\
\text { of } \\
\text { eyes }\end{array}$} & \multirow{2}{*}{$\begin{array}{c}\text { Follow-up, } \\
\text { months }\end{array}$} & \multirow{2}{*}{$\begin{array}{l}\text { Criteria for } \\
\text { Progression }\end{array}$} & \multirow{2}{*}{$\begin{array}{l}\text { UV device/ } \\
\text { Riboflavin }\end{array}$} & \multirow[t]{2}{*}{ Protocol } & \multicolumn{6}{|c|}{ Outcome } \\
\hline & & & & & & & Overall & Pre-op K (D) & $\Delta \mathrm{K}(\mathrm{D})$ & $\Delta$ UCVA & $\triangle \mathrm{BCVA}$ & $\begin{array}{c}\Delta \text { Refraction } \\
\text { (D) }\end{array}$ \\
\hline $\begin{array}{l}\text { Moramarco et } \\
a l, 2015 \text { [66] }\end{array}$ & $\begin{array}{l}\text { Retrospective case } \\
\text { series/Keratoconus }\end{array}$ & 60 & 1 & $\begin{array}{c}\Delta \text { in corneal curvature } \\
\text { in the cone area of } \geq 1.0 \\
D \text { or thinning of }>10 \mu \mathrm{m} \\
\text { in minimal pachymetry } \\
\text { in } 2 \text { consecutive } \\
\text { topography maps/ } 6 \\
\text { months }\end{array}$ & $\begin{array}{c}\text { Avedro KXL I/ } \\
0.1 \% \text { riboflavin with } \\
1 \% \text { HPMC } 10 \mathrm{~min}\end{array}$ & $\begin{array}{c}\text { Pulsed: } \\
30 \mathrm{~mW} / \mathrm{cm}^{2} 8 \\
\mathrm{~min} \\
\text { Continuous: } \\
30 \mathrm{~mW} / \mathrm{cm}^{2} 4 \\
\mathrm{~min}\end{array}$ & $\begin{array}{l}\text { Pulsed accelerated CXL } \\
\text { had a significantly deeper } \\
\text { demarcation line as } \\
\text { compared to continuous } \\
\text { light exposure. }\end{array}$ & \begin{tabular}{|c|} 
Max K \\
(pulsed): $47 \pm 6$ \\
Max K \\
(continuous): \\
$48.6 \pm 3.8$ \\
$(\mathrm{P}>0.05)$
\end{tabular} & - & - & - & - \\
\hline
\end{tabular}

$U V=$ Ultraviolet pre-op = pre-operative $U C V A=$ Uncorrected Visual Acuity $B C V A=$ Best Corrected Visual Acuity $U D V A=$ Uncorrected Distance Visual Acuity

$C D V A=$ Corrected Distance Visual Acuity Kmax = maximum keratometry Kmean $=$ mean keratometry $K=$ Keratometry $S E=$ Spherical Equivalent $C X L=$ cross-linking

$c y l=$ cylinder $D=$ Diopters $M R S E=$ Manifest Refractive Spherical Equivalent $\max =$ maximum paeds $=$ paediatric

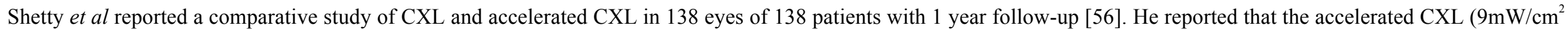
for 10 minutes and $18 \mathrm{~mW} / \mathrm{cm}^{2}$ for 5 minutes) had similar outcomes to standard CXL but the accelerated CXL using $30 \mathrm{~mW} / \mathrm{cm}^{2}$ for $3 \mathrm{minutes}$ was not as efficacious.

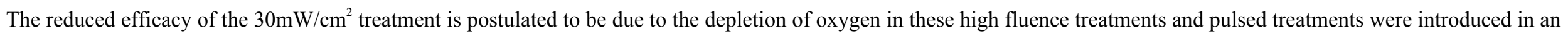




of accelerated CXL to CXL. 


\section{CROSS-LINKING COMBINED WITH REFRACTIVE PROCEDURES FOR THE TREATMENT OF CORNEAL ECTASIA (CXL PLUS)}

Although CXL is effective in stabilizing keratoconus, in many cases, patients are unable to achieve functional vision after CXL and still require rigid contact lens wear. Hence refractive treatments in combination with CXL (CXL plus) have been introduced to provide patients with better visual acuity.

\subsection{Photorefractive Keratectomy (PRK) and CXL}

Kanellopoulos and Binder reported on the first case of topography-guided PRK performed 1 year after CXL for the treatment of keratoconus showing improvement in visual acuity [67]. Subsequently Kanellopuolos reported that simultaneous treatment (PRK followed by CXL) is more effective than sequential treatment (CXL followed 6 months later by PRK) [68] in the visual rehabilitation of keratoconus. Other studies also confirmed the safety and efficacy of simultaneous topography guided PRK and CXL [69 - 77]. Some studies advocate the use of mitomycin C $0.02 \%$ after laser ablation while others do not.

\subsection{Transepithelial Phototherapeutic Keratectomy (PTK) and CXL}

The removal of corneal epithelium in the CXL procedure is replaced with PTK which not only removes epithelium but also regularises the anterior corneal surface [78]. Kymionis et al, in a comparative study showed that epithelial removal using PTK during CXL (Cretan protocol) results in better visual and refractive outcomes than mechanical removal of epithelium [79]. Transepithelial PTK uses the patient's epithelium as a masking agent. At the apex of the cone, epithelium and the anterior stromal surface is removed resulting in a more regularized anterior corneal surface [78].

\subsection{CXL and Intrastromal Corneal Ring Segment (ICRS) Implantation}

Intrastromal Corneal Ring Segment (ICRS) implantation is currently a treatment option for keratoconus and postLASIK ectasia [80 - 82]. However, it does not prevent keratoconus progression and in young patients with progressive keratoconus, CXL may be performed in addition to ICRS to add biomechanical stability. Chan et al reported that ICRS (Intacs) with CXL resulted in better keratoconus improvement than Intacs insertion alone [83]. Coskunseven reported that ICRS implantation followed by CXL resulted in greater keratoconus improvement than CXL followed by ICRS [84]. El Awady reported that CXL has an additive effect after Keraring implantation (Mediphacos, Belo Horizonte, Brazil) [85]. Studies on simultaneous transepithelial ICRS - CXL report that CXL has an additive effect on ICRS [86, 87]. Lam et al. reported a case of post-LASIK ectasia treated with femtosecond laser-assisted ICRS implantation followed by CXL resulting in stabilization of ectasia and improvement in vision [88].

\subsection{CXL and Phakic Intraocular Lens Implantation}

Several case series report on the safety and efficacy of CXL followed by toric Visian ICL [89 - 91]. Similar results were obtained with Artiflex lens implantation 6 months after CXL and toric iris-claw lens implantation (Artiflex: Ophtec BV) [92]

\section{CUSTOMISED CROSS-LINKING}

Kanellopoulos first reported on a case of customized high fluence toric application of transepithelial cross-linking which resulted in a reduction in corneal astigmatism(0.8D) and improvement in the uncorrected visual acuity from 20/40 to 20/25 at 6 months followup [93].

Roy and Dupps demonstrated using three dimensional finite element analysis model that there is differential biomechanical weakening in the area of the cone. They concluded that there is greater efficacy of smaller diameter cone-centric treatments for the reduction of corneal curvature [94]. The Mosaic delivery system (KXL II, Avedro Inc., Waltham, MA, USA) offers customised cross-linking (photorefractive intrastromal cross-linking- PiXL). Initial studies on customised cross-liking report greater corneal regularisation and reduction in maximum keratometry than conventional cross-linking [95 - 97].

Customised cross-linking has recently been used to correct low degrees of refractive error in a patient without keratoconus. Kanellopoulos first described the preliminary results for low myopic correction [98]. Lim et al reported on the results of PiXL for the treatment of low myopia in a cohort of 14 eyes with a 1 year followup [99]. High fluence UV-A irradiation ranging from $10-15 \mathrm{~J} / \mathrm{cm}$ was delivered over a $4.5 \mathrm{~mm}$ central zone. A mean reduction of 0.72 
+/-0.43D was noted at 1 year followup. Kanellopoulos reported on the results of PiXL for hyperopia, with a mean correction of $+0.85 \mathrm{D}[100]$.

\section{COMBINED LASER IN-SITU KERATOMILEUSIS (LASIK) AND ACCELERATED CORNEAL CROSS- LINKING}

LASIK, with the creation of a corneal flap and ablation of corneal tissue weakens the biomechanical strength of the cornea and in susceptible eyes, may predispose to post-LASIK ectasia. In order to strengthen the cornea, accelerated corneal cross-linking is performed simultaneously after the LASIK procedure. Studies have reported that combined laser in-situ Keratomileusis (LASIK) and accelerated corneal cross-linking may confer additional benefits of early refractive and keratometric stability after LASIK, improving the predictability of refractive outcomes in patients. The indications are high myopia corrections, hyperopic corrections, patients with lower residual stromal bed thickness and patients with thin corneas.

LASIK in patients with high myopia has a higher incidence of refractive regression $[101,102]$. Hence the use of simultaneous accelerated CXL and LASIK to stabilize the patient's refraction may be useful particularly in patients with high myopia. In a prospective study comparing 73 LASIK Xtra eyes and 82 LASIK only eyes, Kanellopoulos et al found that $90.4 \%$ of LASIK Xtra eyes had UDVA of $20 / 20$ or better as compared to $85.4 \%$ of LASIK only eyes at postoperative month $12(\mathrm{p}=0.042)$ [103]. Similar findings were also shown in another prospective study comparing LASIK Xtra in one eye and LASIK only in the fellow eye over a 12-month period [104].

Kanellopoulos et al also reported that corneal keratometry measurements were stable for LASIK Xtra eyes and slightly regressing in LASIK only eyes $(p=0.039)$ [103]. Subsequently, Kanellopoulos also reported a statistically significant reduction in regression in a 2 year analysis of LASIK-CXL for high myopia compared to the LASIK only group [105]. LASIK and accelerated cross-linking for hyperopia also showed better refractive stability and less regression than LASIK only [106]. Another study by Kanellopoulos found significantly less epithelial thickening in the LASIK and accelerated CXL group compared to the LASIK only group. This could possibly explain the differences in the refractive stability between the 2 groups [107]. LASIK and accelerated CXL has been shown to be comparable to LASIK only in terms of safety, as evidenced by similar loss of corrected visual acuity in both groups [103, 105].

Studies on LASIK and accelerated cross-linking report the use of different levels of UV irradiance, energy levels and illumination times [104, 105, 108, 109]. The energy levels vary from $1.8 \mathrm{~J} / \mathrm{cm}^{2}$ to $5.4 \mathrm{~J} / \mathrm{cm}^{2}$. It is postulated energy settings may be lower $\left(1.8 \mathrm{~J} / \mathrm{cm}^{2}\right)$ than conventional cross-linking treatment for keratoconus $\left(5.4 \mathrm{~J} / \mathrm{cm}^{2}\right)$ since eyes undergoing LASIK and accelerated cross-linking are normal eyes.

One of the goals of performing LASIK with accelerated CXL is reducing the risk of post-LASIK ectasia. A review of the literature of eyes that had undergone LASIK and accelerated cross-linking with at least 2 years follow-up showed no report of post-LASIK ectasia supporting the claim that LASIK with accelerated CXL may prevent post-LASIK ectasia [110]. However, post-LASIK ectasia has been shown to develop as long as 5 to 10 years postoperatively. Hence these reports are not sufficient to make this conclusion and further long term studies are warranted.

\section{CROSS-LINKING FOR THE TREATMENT OF INFECTIVE KERATITIS (PACK-CXL) (TABLE 5)}

Infectious keratitis is a serious, sight-threatening condition that can result from bacterial, viral, fungal or protozoal infection. Standard treatment for infectious keratitis involves both systemic and topical antimicrobial therapy. However, the effectiveness of this treatment depends on microbial sensitivity to the drug as well as severity of the disease process. Infections not responding to antimicrobial therapy may require therapeutic keratoplasty (lamellar or penetrating). Corneal Collagen cross-Linking (CXL), or Photo Activated Chromophore for Keratitis (PACK-CXL) has been investigated as a possible alternative treatment for infectious keratitis. CXL treatment stiffens the corneal stroma through the effect of photo-activated riboflavin on collagen fibers. This makes the cornea more resistant to enzymatic degradation by microbes, thus reducing the progression of corneal melting [111,112]. Also, in CXL, riboflavin enters an excited state and reacts with ambient oxygen to create Reactive Oxygen Species (ROS). These ROS cause cell death by damaging intracellular components. Microorganisms are also killed by ROS damage to microbe DNA and cytoplasmic membrane, resulting in leakage of cellular contents and inactivation of enzymes and membrane transport systems [113]. 
Table 5. Summary of outcomes for cross-linking in infectious keratitis.

\begin{tabular}{|c|c|c|c|c|c|c|}
\hline Study & Study design & Indication & $\begin{array}{l}\text { No. of } \\
\text { Eyes }\end{array}$ & $\begin{array}{c}\text { Follow-up, } \\
\text { month }\end{array}$ & Other treatment & Findings \\
\hline Iseli et al, 2008 [114] & Prospective case series & $\begin{array}{c}\text { Infectious keratitis unresponsive to } \\
\text { antibiotics }\end{array}$ & 5 & $1-9$ & $\begin{array}{l}\text { Topical and systemic } \\
\text { antibiotic therapy }\end{array}$ & $\begin{array}{c}\text { In all cases, progression of corneal melting was halted. Emergency keratoplasty was } \\
\text { not required in any of the cases. }\end{array}$ \\
\hline $\begin{array}{l}\text { Micelli Ferrari et al, } \\
2009[115]\end{array}$ & Case report & $\begin{array}{c}\text { Bacterial keratitis caused by Gram } \\
\text { negative E. coli }\end{array}$ & 1 & 1 & $\begin{array}{l}\text { Topical and systemic } \\
\text { antimicrobial therapy }\end{array}$ & Corneal edema almost completely resolved, corneal ulceration healed after 1 month \\
\hline $\begin{array}{l}\text { Makdoumi et al, } \\
2010[116]\end{array}$ & Prospective case series & $\begin{array}{c}\text { Infectious keratitis associated with } \\
\text { corneal melting }\end{array}$ & 7 & $1-6$ & $\begin{array}{l}\text { Topical antibiotics (all } \\
\text { except 1) }\end{array}$ & Corneal melting arrested and complete epithelialisation was achieved in all cases. \\
\hline $\begin{array}{l}\text { Moren et al, } 2010 \\
\quad[117]\end{array}$ & Case report & Suspected acanthamoeba keratitis & 1 & 9 & Broad-spectrum antibiotics & $\begin{array}{c}\text { Rapid decrease of pain and necrotic material. Corneal reepithelialisation started } \\
\text { within a few days and completed within } 1 \text { month. Complete wound healing after } 2 \\
\text { months. BCVA improved from 20/1000 to 20/30 after } 9 \text { months. }\end{array}$ \\
\hline $\begin{array}{l}\text { Khan et al, } 2011 \\
\quad[118]\end{array}$ & Interventional case series & $\begin{array}{l}\text { Acanthamoeba keratitis } \\
\text { unresponsive to treatment }\end{array}$ & 3 & 2 & $\begin{array}{l}\text { Multidrug conventional } \\
\text { therapy }\end{array}$ & $\begin{array}{l}\text { Rapid reduction in symptoms and decreased ulcer size after the first treatment } \\
\text { session. Progress of improvement slowed after } 1 \text { to } 3 \text { weeks but renewed after the } \\
\text { second application. Ulcers closed within } 3 \text { to } 7 \text { weeks of first application. In } 2 \\
\text { patients, penetrating keratoplasty was subsequently performed for residual dense } \\
\text { corneal scars. }\end{array}$ \\
\hline $\begin{array}{c}\text { Anwar et al, } 2011 \\
{[119]}\end{array}$ & $\begin{array}{l}\text { Retrospective case } \\
\text { reports }\end{array}$ & $\begin{array}{c}\text { Infective keratitis unresponsive to } \\
\text { antimicrobial therapy }\end{array}$ & 2 & - & Antimicrobial therapy & $\begin{array}{l}\text { Rapid resolution of infective keratitis, leaving residual stromal scarring. } 1 \text { patient } \\
\text { required penetrating keratoplasty for residual dense corneal scars. }\end{array}$ \\
\hline $\begin{array}{l}\text { Makdoumi et al, } \\
\quad 2012[120]\end{array}$ & $\begin{array}{l}\text { Prospective non- } \\
\text { randomised study }\end{array}$ & Bacterial keratitis & 16 & - & $\begin{array}{c}\text { Antibiotics only given for } 2 \\
\text { out of } 16 \text { eyes }\end{array}$ & $\begin{array}{l}\text { All eyes responded to photochemical treatment. Improved symptoms, reduced } \\
\text { inflammation. Epithelial healing achieved. One patient required human amniotic } \\
\text { membrane transplant. }\end{array}$ \\
\hline $\begin{array}{c}\text { Price } e \text { et al, } 2012 \\
\quad[125]\end{array}$ & $\begin{array}{l}\text { Prospective, dual-center, } \\
\text { interventional case series }\end{array}$ & $\begin{array}{l}\text { Infective keratitis (bacterial, } \\
\text { fungal, protozoan, viral) }\end{array}$ & 40 & - & $\begin{array}{l}\text { Standard antibiotic } \\
\text { treatment, } 7 \text { patients had } \\
\text { previous keratoplasty }\end{array}$ & $\begin{array}{l}\text { Keratitis did not resolve in } 6 \text { cases and penetrating keratoplasty was needed. CXL } \\
\text { should be avoided in eyes with prior herpes simplex. CXL appeared most effective } \\
\text { when infection depth was limited. Success higher for bacterial than fungal infections. }\end{array}$ \\
\hline $\begin{array}{c}\text { Kymionis et al, } 2012 \\
{[121]}\end{array}$ & Case report & $\begin{array}{c}\text { Intractable post-laser keratitis due } \\
\text { to atypical mycobacteria }\end{array}$ & 1 & 3 & Maximum antibiotic therapy & $\begin{array}{c}\text { All infiltrates and stromal edema resolved after } 1 \text { week. UDVA improved from } \\
\text { counting fingers at } 3 \text { meters to } 20 / 35 .\end{array}$ \\
\hline Li et al, 2013 [122] & Prospective case series & $\begin{array}{l}\text { Fungal keratitis unresponsive to } \\
\text { treatment }\end{array}$ & 8 & - & Topical antibiotics & $\begin{array}{l}\text { No complications noted. Hypopyon disappeared in all cases between } 3 \text { to } 11 \text { days } \\
\text { after CXL. Healing of corneal epithelium and ulcer was achieved between } 3 \text { and } 8 \\
\text { days after CXL. }\end{array}$ \\
\hline $\begin{array}{l}\text { Arance-Gil et al, } \\
\quad 2014[131]\end{array}$ & Case report & $\begin{array}{c}\text { Acanthamoeba keratitis } \\
\text { unresponsive to medical treatment }\end{array}$ & 1 & 9 & Medical treatment & $\begin{array}{l}\text { After CXL, symptoms and corneal appearance improved significantly but the ulcer } \\
\text { did not heal completely. Patient required amniotic membrane transplantation and } \\
\text { penetrating keratoplasty. }\end{array}$ \\
\hline $\begin{array}{l}\text { Saglk et al, } 2013 \\
{[123]}\end{array}$ & Case report & $\begin{array}{l}\text { Suspected fungal keratitis } \\
\text { unresponsive to treatment }\end{array}$ & 1 & 6 & Extensive medical treatment & $\begin{array}{c}\text { Epithelial defect disappeared and stromal infiltrate stayed inactive from } 1 \text { week to } 6 \\
\text { months after the second treatment. }\end{array}$ \\
\hline $\begin{array}{l}\text { Shetty et al, } 2014 \\
\quad[127]\end{array}$ & Prospective case series & $\begin{array}{l}\text { Microbial keratitis (bacterial and } \\
\text { fungal) }\end{array}$ & 15 & - & Antibiotics / antifungals & $\begin{array}{l}\text { 6/9 patients with bacterial keratitis and } 3 / 6 \text { patients with fungal keratitis resolved after } \\
\text { CXL treatment. Patients with deep stromal keratitis or endothelial plaque failed to } \\
\text { resolve. }\end{array}$ \\
\hline
\end{tabular}




\begin{tabular}{|c|c|c|c|c|c|c|}
\hline Study & Study design & Indication & $\begin{array}{l}\text { No. of } \\
\text { Eyes }\end{array}$ & $\begin{array}{c}\text { Follow-up, } \\
\text { month }\end{array}$ & Other treatment & Findings \\
\hline $\begin{array}{c}\text { Tabibian et al, } 2014 \\
{[124]}\end{array}$ & Case report & $\begin{array}{l}\text { Atypical fungal keratitis } \\
\text { (Aureobasidium pullulans) }\end{array}$ & 1 & - & None & $\begin{array}{c}\text { Corneal epithelium closed completely within } 3 \text { days and infiltrate was completely } \\
\text { eradicated. }\end{array}$ \\
\hline Said et al, 2014 [128] & Prospective clinical trial & $\begin{array}{l}\text { Infectious keratitis with corneal } \\
\text { melting (bacterial, fungal, } \\
\text { amoebic) }\end{array}$ & $\begin{array}{l}40 ; 21 \\
\text { case, } 19 \\
\text { control }\end{array}$ & - & $\begin{array}{l}\text { Case: Antibiotics }+ \text { CXL } \\
\text { Control: Antibiotics only }\end{array}$ & $\begin{array}{l}\text { Average healing time was } 39.76+/-18.22 \text { (PACK-CXL) and } 46.05+/-27.44 \\
\text { (control). CDVA after healing was 1.64 +/- } 0.62 \text { (PACK-CXL) and } 1.67+/-0.48 \\
\text { (control). The PACK-CXL group had a bigger corneal ulceration width and length. }\end{array}$ \\
\hline $\begin{array}{l}\text { Vajpayee } \text { et al, } 2015 \\
{[129]}\end{array}$ & $\begin{array}{l}\text { Retrospective case-file } \\
\text { analysis }\end{array}$ & Moderate mycotic keratitis & $\begin{array}{l}41 ; 20 \\
\text { case, } 21 \\
\text { control }\end{array}$ & - & $\begin{array}{l}\text { Case: Antibiotics }+ \text { CXL } \\
\text { Control: Antibiotics only }\end{array}$ & $\begin{array}{l}\text { Average healing time and final BCVA were similar in both groups. The additional } \\
\text { CXL treatment did not have any advantage over medical treatment. }\end{array}$ \\
\hline $\begin{array}{c}\text { Uddaraju et al, } 2015 \\
{[130]}\end{array}$ & Randomised clinical trial & $\begin{array}{l}\text { Nonresolving deep stromal fungal } \\
\text { keratitis }\end{array}$ & $\begin{array}{l}13 ; 6 \\
\text { case, } 7 \\
\text { control }\end{array}$ & - & $\begin{array}{l}\text { Case: Antibiotics }+ \text { CXL } \\
\text { Control: Antibiotics only }\end{array}$ & $\begin{array}{l}\text { The trial was stopped due to a marked difference in the rate of perforation between } \\
\text { the } 2 \text { groups. The CXL group had a significantly higher rate of perforation. }\end{array}$ \\
\hline $\begin{array}{c}\text { Bamdad et al, } 2015 \\
{[132]}\end{array}$ & $\begin{array}{c}\text { Prospective randomised } \\
\text { clinical study }\end{array}$ & Moderate bacterial corneal ulcers & $\begin{array}{c}32 ; 16 \\
\text { case, } 16 \\
\text { control }\end{array}$ & 0.5 & $\begin{array}{l}\text { Case: Antibiotics }+ \text { CXL } \\
\text { Control: Antibiotics only }\end{array}$ & $\begin{array}{c}\text { Mean treatment duration was } 17.2+/-4.1 \text { days in the case group and } 24.7+/-5.5 \text { days } \\
\text { in the control group. Epithelial defects were smaller in the case group at } 7 \text { and } 14 \\
\text { davs. }\end{array}$ \\
\hline
\end{tabular}

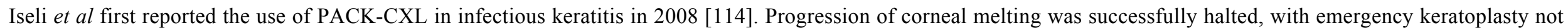





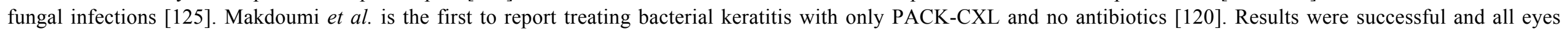
responded to the treatment, with only 2 eyes requiring additional antibiotics and 1 eye requiring an amniotic membrane transplant.

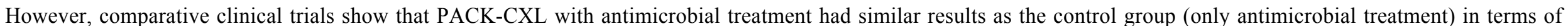

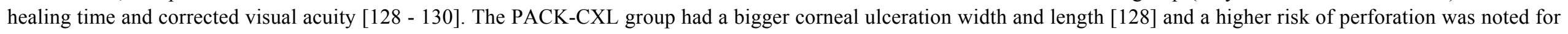
deep fungal keratitis [130]. 


\section{OTHER APPLICATIONS OF CROSS-LINKING}

Corneal cross-linking can be employed to prevent further progression in pellucid marginal degeneration(PMD) which is considered a variant of keratoconus. Several studies report on its safety and efficacy [133, 134] [135]. Additionally Kymionis performed simultaneous photorefractive keratectomy and CXL in a both eyes of a patient with PMD resulting in significant improvement in the corneal topography measurements and visual acuity [136].

Corneal cross-linking has also been used as a treatment for pain relief in bullous keratopathy. Sharma et al reported on cross-linking treatment in 50 eyes with bullous keratopathy and concluded that the pain relief achieved was temporary with corneal bullae recurring in $44 \%$ of the cases. No long term improvement in visual acuity was seen [137]. Kozobolis et al reported on CXL as an adjunctive treatment for patients with combined bullous keratopathy and infective keratitis [138].

Mukherjee et al performed an animal model evaluation of cross-linking donor corneas for penetrating keratoplasty and concluded that it reduces intraoperative induced astigmatism and aberrations in an animal model [139]. Ting et al [140] conducted a randomised controlled trial to investigate whether donor corneas pre-treated with cross-linking reduced myopic refractive errors for keratoconic eyes after penetrating keratoplasty. At 3 years followup, they found significantly improved corrected visual acuity, reduced Kmax and keratometric astigmatism in the CXL treated group.

Crosslinked corneal tissue has been shown to have stiffer biomechanical properties and to be more resistant to degradation by collagenolytic enzymes. Robert et al reported on cross-linking of the Boston keratoprosthesis donor carrier to prevent corneal melting in a patient with post KPro corneal melt. The patient maintained his visual acuity and showed no evidence of corneal thinning or melt in the first postoperative year [141].

\section{CROSS-LINKING COMPLICATIONS}

Complications of corneal cross-linking include corneal haze, corneal scarring, infective keratitis, sterile infiltrates, delayed epithelial healing, failure of treatment, excessive corneal flattening with hyperopic shift and endothelial failure [142]

Anterior corneal haze occurs frequently and usually appears 1-2 months after cross-linking. It is usually transient and clears by 6 to 12 months [142]. Permanent stromal scarring [143] may occur and the incidence has been reported to be as high as $8.6 \%$ in one series [144]. It may also be more prevalent in eyes receiving simultaneous PRK followed by CXL [145]. Infective keratitis after cross-linking is rare. Shetty et al reported an incidence of $0.0017 \%$ (4 out of 2350 patients) with all 4 cases treated with the epithelium-off technique [146]. Sterile infiltrates present in the early postoperative period (days to weeks) and usually resolve with topical steroid medication [147]. Other uncommon complications include corneal melting associated with atopic eye disease [148] and reactivation of herpetic keratitis [149] Cross-linking should be avoided in patients with previous herpetic eye disease and atopic eye disease should be controlled prior to CXL.

Long-term studies show that progression of keratoconus after cross-linking may occur in about $8 \%$ of cases [23, 24, 150]. Hence it is necessary to counsel patients preoperatively about the various potential side-effects and also about the failure rate of the procedure.

Corneal endothelial damage may occur if the safety limits regarding corneal thickness to prevent endothelial toxicity are not adhered to. Sharma et al reported a 1.4\% incidence of persistent endothelial failure in 350 eyes treated with the standard epithelium-off protocol although the safety limit of corneal thicknesses of greater than 400um (epithelium-off) were adhered to [151]. This could be due to intraoperative stromal dehydration resulting in stromal thinning, lack of homogeneity and focusing/alignment issues of the UV devices.

Although limbal stem cell damage after CXL has been shown in cadaveric eyes [152], long term studies show no evidence of limbal dysfunction [18, 19, 21].

\section{CONCLUSION}

Corneal cross-linking is a unique procedure with an expanding list of indications from the treatment of corneal ectasia to infective keratitis. While the standard Dresden protocol is established as the gold standard treatment for progressive keratoconus, the more recent protocols may require further refinements, investigations and long-term studies. New indications and treatment protocols are also being developed and we look forward to these treatments in the future. 


\section{CONSENT OF PUBLICATION}

Not applicable.

\section{CONFLICT OF INTERESTS}

The authors declare no conflict of interest, financial or otherwise.

\section{ACKNOWLEDGEMENTS}

Decleared none.

\section{REFERENCES}

[1] Wollensak G, Spoerl E, Seiler T. Riboflavin/ultraviolet-a-induced collagen crosslinking for the treatment of keratoconus. Am J Ophthalmol 2003; 135(5): 620-7. [http://dx.doi.org/10.1016/S0002-9394(02)02220-1] [PMID: 12719068]

[2] O'Brart DP, Chan E, Samaras K, Patel P, Shah SP. A randomised, prospective study to investigate the efficacy of riboflavin/ultraviolet A (370 $\mathrm{nm}$ ) corneal collagen cross-linkage to halt the progression of keratoconus. Br J Ophthalmol 2011; 95(11): $1519-24$. [http://dx.doi.org/10.1136/bjo.2010.196493] [PMID: 21349938]

[3] Wittig-Silva C, Whiting M, Lamoureux E, Lindsay RG, Sullivan LJ, Snibson GR. A randomized controlled trial of corneal collagen crosslinking in progressive keratoconus: Preliminary results. J Refract Surg 2008; 24(7): S720-5. [PMID: 18811118]

[4] Wittig-Silva C, Chan E, Islam FM, Wu T, Whiting M, Snibson GR. A randomized, controlled trial of corneal collagen cross-linking in progressive keratoconus: Three-year results. Ophthalmology 2014; 121(4): 812-21. [http://dx.doi.org/10.1016/j.ophtha.2013.10.028] [PMID: 24393351]

[5] Spoerl E, Mrochen M, Sliney D, Trokel S, Seiler T. Safety of UVA-riboflavin cross-linking of the cornea. Cornea 2007; 26(4): 385-9. [http://dx.doi.org/10.1097/ICO.0b013e3180334f78] [PMID: 17457183]

[6] Jain V, Gazali Z, Bidayi R. Isotonic riboflavin and HPMC with accelerated cross-linking protocol. Cornea 2014; 33(9): 910-3. [http://dx.doi.org/10.1097/ICO.0000000000000188] [PMID: 25014154]

[7] Seiler T, Hafezi F. Corneal cross-linking-induced stromal demarcation line. Cornea 2006; 25(9): 1057-9. [http://dx.doi.org/10.1097/01.ico.0000225720.38748.58] [PMID: 17133053]

[8] Cho KS, Lee EH, Choi JS, Joo CK. Reactive oxygen species-induced apoptosis and necrosis in bovine corneal endothelial cells. Invest Ophthalmol Vis Sci 1999; 40(5): 911-9. [PMID: 10102288]

[9] Kanellopoulos AJ, Asimellis G. Introduction of quantitative and qualitative cornea optical coherence tomography findings induced by collagen cross-linking for keratoconus: A novel effect measurement benchmark. Clin Ophthalmol 2013; 7: 329-35. [http://dx.doi.org/10.2147/OPTH.S40455] [PMID: 23440024]

[10] Vinciguerra P, Albè E, Trazza S, et al. Refractive, topographic, tomographic, and aberrometric analysis of keratoconic eyes undergoing corneal cross-linking. Ophthalmology 2009; 116(3): 369-78. [http://dx.doi.org/10.1016/j.ophtha.2008.09.048] [PMID: 19167087]

[11] Vinciguerra P, Albè E, Trazza S, Seiler T, Epstein D. Intraoperative and postoperative effects of corneal collagen cross-linking on progressive keratoconus. Arch Ophthalmol 2009; 127(10): 1258-65. [http://dx.doi.org/10.1001/archophthalmol.2009.205] [PMID: 19822840]

[12] Caporossi A, Baiocchi S, Mazzotta C, Traversi C, Caporossi T. Parasurgical therapy for keratoconus by riboflavin-ultraviolet type A rays induced cross-linking of corneal collagen: Preliminary refractive results in an Italian study. J Cataract Refract Surg 2006; 32(5): 837-45. [http://dx.doi.org/10.1016/j.jcrs.2006.01.091] [PMID: 16765803]

[13] Fournié P, Galiacy S, Arné JL, Malecaze F. Corneal collagen cross-linking with ultraviolet-A light and riboflavin for the treatment of progressive keratoconus. J Fr Ophtalmol 2009; 32(1): 1-7. [PMID: 19515306]

[14] Henriquez MA, Izquierdo L Jr, Bernilla C, Zakrzewski PA, Mannis M. Riboflavin/Ultraviolet A corneal collagen cross-linking for the treatment of keratoconus: Visual outcomes and Scheimpflug analysis. Cornea 2011; 30(3): 281-6. [http://dx.doi.org/10.1097/ICO.0b013e3181 eeaea1] [PMID: 21045662]

[15] Agrawal VB. Corneal collagen cross-linking with riboflavin and ultraviolet - A light for keratoconus: results in Indian eyes. Indian J Ophthalmol 2009; 57(2): 111-4.

[http://dx.doi.org/10.4103/0301-4738.44515] [PMID: 19237783]

[16] Arbelaez MC, Sekito MB, Vidal C, Choudhury SR. Collagen cross-linking with riboflavin and ultraviolet-A light in keratoconus: One-year results. Oman J Ophthalmol 2009; 2(1): 33-8. [http://dx.doi.org/10.4103/0974-620X.48420] [PMID: 21234222] 
[17] Coskunseven E, Jankov MR II, Hafezi F. Contralateral eye study of corneal collagen cross-linking with riboflavin and UVA irradiation in patients with keratoconus. J Refract Surg 2009; 25(4): 371-6. [http://dx.doi.org/10.3928/1081597X-20090401-02] [PMID: 19431928]

[18] Raiskup-Wolf F, Hoyer A, Spoerl E, Pillunat LE. Collagen crosslinking with riboflavin and ultraviolet-A light in keratoconus: Long-term results. J Cataract Refract Surg 2008; 34(5): 796-801. [http://dx.doi.org/10.1016/j.jcrs.2007.12.039] [PMID: 18471635]

[19] O’Brart DP, Kwong TQ, Patel P, McDonald RJ, O’Brart NA. Long-term follow-up of riboflavin/ultraviolet A (370 nm) corneal collagen cross-linking to halt the progression of keratoconus. Br J Ophthalmol 2013; 97(4): 433-7. [http://dx.doi.org/10.1136/bjophthalmol-2012-302556] [PMID: 23385632]

[20] Hashemi H, Seyedian MA, Miraftab M, Fotouhi A, Asgari S. Corneal collagen cross-linking with riboflavin and ultraviolet a irradiation for keratoconus: long-term results. Ophthalmology 2013; 120(8): 1515-20. [http://dx.doi.org/10.1016/j.ophtha.2013.01.012] [PMID: 23583165]

[21] Caporossi A, Mazzotta C, Baiocchi S, Caporossi T. Long-term results of riboflavin ultraviolet a corneal collagen cross-linking for keratoconus in Italy: the Siena eye cross study. Am J Ophthalmol 2010; 149(4): 585-93. [http://dx.doi.org/10.1016/j.ajo.2009.10.021] [PMID: 20138607]

[22] Uçakhan OO, Bayraktutar BN, Saglik A. Pediatric corneal collagen cross-Linking: Long-term follow-up of visual, refractive, and topographic outcomes. Cornea 2016; 35(2): 162-8. [http://dx.doi.org/10.1097/ICO.0000000000000702] [PMID: 26655483]

[23] Raiskup F, Theuring A, Pillunat LE, Spoerl E. Corneal collagen crosslinking with riboflavin and ultraviolet-A light in progressive keratoconus: Ten-year results. J Cataract Refract Surg 2015; 41(1): 41-6. [PMID: 25532633]

[24] O'Brart DP, Patel P, Lascaratos G, et al. Corneal cross-linking to halt the progression of keratoconus and corneal ectasia: Seven-year followup. Am J Ophthalmol 2015; 160(6): 1154-63. [http://dx.doi.org/10.1016/j.ajo.2015.08.023] [PMID: 26307513]

[25] Hafezi F, Kanellopoulos J, Wiltfang R, Seiler T. Corneal collagen crosslinking with riboflavin and ultraviolet A to treat induced keratectasia after laser in situ keratomileusis. J Cataract Refract Surg 2007; 33(12): 2035-40. [http://dx.doi.org/10.1016/j.jcrs.2007.07.028] [PMID: 18053900]

[26] Salgado JP, Khoramnia R, Lohmann CP, Winkler von Mohrenfels C. Corneal collagen crosslinking in post-LASIK keratectasia. Br J Ophthalmol 2011; 95(4): 493-7. [http://dx.doi.org/10.1136/bjo.2010.179424] [PMID: 20679077]

[27] Vinciguerra P, Camesasca FI, Albè E, Trazza S. Corneal collagen cross-linking for ectasia after excimer laser refractive surgery: 1-year results. J Refract Surg 2010; 26(7): 486-97.

[http://dx.doi.org/10.3928/1081597X-20090910-02] [PMID: 19772221]

[28] Hersh PS, Greenstein SA, Fry KL. Corneal collagen crosslinking for keratoconus and corneal ectasia: One-year results. J Cataract Refract Surg 2011; 37(1): 149-60. [http://dx.doi.org/10.1016/j.jcrs.2010.07.030] [PMID: 21183110]

[29] Richoz O, Mavrakanas N, Pajic B, Hafezi F. Corneal collagen cross-linking for ectasia after LASIK and photorefractive keratectomy: Longterm results. Ophthalmology 2013; 120(7): 1354-9. [http://dx.doi.org/10.1016/j.ophtha.2012.12.027] [PMID: 23582990]

[30] Lang SJ, Messmer EM, Geerling G, et al. Prospective, randomized, double-blind trial to investigate the efficacy and safety of corneal crosslinking to halt the progression of keratoconus. BMC Ophthalmol 2015; 15: 78.

[http://dx.doi.org/10.1186/s12886-015-0070-7] [PMID: 26194634]

[31] Vinciguerra P, Albé E, Frueh BE, Trazza S, Epstein D. Two-year corneal cross-linking results in patients younger than 18 years with documented progressive keratoconus. Am J Ophthalmol 2012; 154(3): 520-6. [http://dx.doi.org/10.1016/j.ajo.2012.03.020] [PMID: 22633357]

[32] Ivarsen A, Hjortdal J. Collagen cross-linking for advanced progressive keratoconus. Cornea 2013; 32 (7): $903-6$. [http://dx.doi.org/10.1097/ICO.0b013e31828321dd] [PMID: 23449485]

[33] Chatzis N, Hafezi F. Progression of keratoconus and efficacy of pediatric [corrected] corneal collagen cross-linking in children and adolescents. J Refract Surg 2012; 28(11): 753-8. [http://dx.doi.org/10.3928/1081597X-20121011-01] [PMID: 23347367]

[34] Arora R, Gupta D, Goyal JL, Jain P. Results of corneal collagen cross-linking in pediatric patients. J Refract Surg 2012; $28(11)$ : 759-62. [http://dx.doi.org/10.3928/1081597X-20121011-02] [PMID: 23347368]

[35] Caporossi A, Mazzotta C, Baiocchi S, Caporossi T, Denaro R, Balestrazzi A. Riboflavin-UVA-induced corneal collagen cross-linking in pediatric patients. Cornea 2012; 31(3): 227-31. [http://dx.doi.org/10.1097/ICO.0b013e31822159f6] [PMID: 22420024]

[36] Buzzonetti L, Petrocelli G. Transepithelial corneal cross-linking in pediatric patients: early results. J Refract Surg 2012; $28(11)$ : 763-7. [http://dx.doi.org/10.3928/1081597X-20121011-03] [PMID: 23347369] 
[37] Filippello M, Stagni E, O’Brart D. Transepithelial corneal collagen crosslinking: bilateral study. J Cataract Refract Surg 2012; 38(2): 283-91. [http://dx.doi.org/10.1016/j.jcrs.2011.08.030] [PMID: 22104644]

[38] Salman AG. Transepithelial corneal collagen crosslinking for progressive keratoconus in a pediatric age group. J Cataract Refract Surg 2013; 39(8): 1164-70

[http://dx.doi.org/10.1016/j.jcrs.2013.03.017] [PMID: 23790531]

[39] Magli A, Forte R, Tortori A, Capasso L, Marsico G, Piozzi E. Epithelium-off corneal collagen cross-linking versus transepithelial crosslinking for pediatric keratoconus. Cornea 2013; 32(5): 597-601. [http://dx.doi.org/10.1097/ICO.0b013e31826cf32d] [PMID: 23132450]

[40] Caporossi A, Mazzotta C, Paradiso AL, Baiocchi S, Marigliani D, Caporossi T. Transepithelial corneal collagen crosslinking for progressive keratoconus: 24-month clinical results. J Cataract Refract Surg 2013; 39(8): 1157-63. [http://dx.doi.org/10.1016/j.jcrs.2013.03.026] [PMID: 23790530]

[41] Al Fayez MF, Alfayez S, Alfayez Y. Transepithelial versus epithelium-off corneal collagen cross-linking for progressive keratoconus: A prospective randomized controlled trial. Cornea 2015; 34(Suppl. 10): S53-6. [http://dx.doi.org/10.1097/ICO.0000000000000547] [PMID: 26266436]

[42] Soeters N, Wisse RP, Godefrooij DA, Imhof SM, Tahzib NG. Transepithelial versus epithelium-off corneal cross-linking for the treatment of progressive keratoconus: A randomized controlled trial Am J Ophthalmol. 2015;159(5):821-8 e3. [http://dx.doi.org/10.1016/j.ajo.2015.02.005]

[43] Bikbova G, Bikbov M. Transepithelial corneal collagen cross-linking by iontophoresis of riboflavin. Acta Ophthalmol 2014; 92(1): e30-4. [http://dx.doi.org/10.1111/aos.12235] [PMID: 23848196]

[44] Vinciguerra P, Randleman JB, Romano V, et al. Transepithelial iontophoresis corneal collagen cross-linking for progressive keratoconus: initial clinical outcomes. J Refract Surg 2014; 30(11): 746-53. [http://dx.doi.org/10.3928/1081597X-20141021-06] [PMID: 25375847]

[45] Buzzonetti L, Petrocelli G, Valente P, Iarossi G, Ardia R, Petroni S. Iontophoretic transepithelial corneal cross-linking to halt keratoconus in pediatric cases: 15-month follow-up. Cornea 2015; 34(5): 512-5. [http://dx.doi.org/10.1097/ICO.0000000000000410] [PMID: 25789695]

[46] Magli A, Chiariello Vecchio E, Carelli R, Piozzi E, Di Landro F, Troisi S. Pediatric keratoconus and iontophoretic corneal crosslinking: Refractive and topographic evidence in patients underwent general and topical anesthesia, 18 months of follow-up. Int Ophthalmol 2016; 36(4): 585-90.

[http://dx.doi.org/10.1007/s10792-015-0166-3] [PMID: 26704375]

[47] Leccisotti A, Islam T. Transepithelial corneal collagen cross-linking in keratoconus. J Refract Surg 2010; $26(12)$ : 942-8. [http://dx.doi.org/10.3928/1081597X-20100212-09] [PMID: 20166621]

[48] Koppen C, Wouters K, Mathysen D, Rozema J, Tassignon MJ. Refractive and topographic results of benzalkonium chloride-assisted transepithelial crosslinking. J Cataract Refract Surg 2012; 38(6): 1000-5. [http://dx.doi.org/10.1016/j.jcrs.2012.01.024] [PMID: 22624899]

[49] Bunsen RRH. Photochemische Untersuchungen Poggendorff's Annalen 1855; 96: 373-94.

[50] Wernli J, Schumacher S, Spoerl E, Mrochen M. The efficacy of corneal cross-linking shows a sudden decrease with very high intensity UV light and short treatment time. Invest Ophthalmol Vis Sci 2013; 54(2): 1176-80 [http://dx.doi.org/10.1167/iovs.12-11409] [PMID: 23299484]

[51] Kanellopoulos AJ. Long term results of a prospective randomized bilateral eye comparison trial of higher fluence, shorter duration ultraviolet A radiation, and riboflavin collagen cross linking for progressive keratoconus. Clin Ophthalmol 2012; 6: 97-101. [http://dx.doi.org/10.2147/OPTH.S27170] [PMID: 22275813]

[52] Gatzioufas Z, Richoz O, Brugnoli E, Hafezi F. Safety profile of high-fluence corneal collagen cross-linking for progressive keratoconus: preliminary results from a prospective cohort study. J Refract Surg 2013; 29(12): 846-8. [http://dx.doi.org/10.3928/1081597X-20131023-03] [PMID: 24168788]

[53] Tomita M, Mita M, Huseynova T. Accelerated versus conventional corneal collagen crosslinking. J Cataract Refract Surg 2014; 40(6): 1013-20.

[http://dx.doi.org/10.1016/j.jcrs.2013.12.012] [PMID: 24857442]

[54] Kymionis GD, Tsoulnaras KI, Grentzelos MA, et al. Corneal stroma demarcation line after standard and high-intensity collagen crosslinking determined with anterior segment optical coherence tomography. J Cataract Refract Surg 2014; 40(5): 736-40. [http://dx.doi.org/10.1016/j.jcrs.2013.10.029] [PMID: 24630796]

[55] Kymionis GD, Tsoulnaras KI, Grentzelos MA, Liakopoulos DA, Tsakalis NG, Blazaki SV, et al. Evaluation of corneal stromal demarcation line depth following standard and a modified-accelerated collagen cross-linking protocol Am J Ophthalmol. 2014;158(4):671-5 e1. [http://dx.doi.org/10.1016/j.ajo.2014.07.005]

[56] Shetty R, Pahuja NK, Nuijts RM, et al. Current protocols of corneal collagen cross-linking: Visual, refractive, and tomographic outcomes. Am J Ophthalmol 2015; 160(2): 243-9.

[http://dx.doi.org/10.1016/j.ajo.2015.05.019] [PMID: 26008626] 
[57] Mazzotta C, Traversi C, Paradiso AL, Latronico ME, Rechichi M. Pulsed light accelerated crosslinking versus continuous light accelerated crosslinking: One-Year results. J Ophthalmol 2014; $2014: 604731$. [http://dx.doi.org/10.1155/2014/604731] [PMID: 25165576]

[58] Sherif AM. Accelerated versus conventional corneal collagen cross-linking in the treatment of mild keratoconus: a comparative study. Clin Ophthalmol 2014; 8: 1435-40. [http://dx.doi.org/10.2147/OPTH.S59840] [PMID: 25120349]

[59] Ng AL, Chan TC, Cheng AC. Conventional versus accelerated corneal collagen cross-linking in the treatment of keratoconus. Clin Experiment Ophthalmol 2016; 44(1): 8-14.

[http://dx.doi.org/10.1111/ceo.12571] [PMID: 26140309]

[60] Chow VW, Chan TC, Yu M, Wong VW, Jhanji V. One-year outcomes of conventional and accelerated collagen crosslinking in progressive keratoconus. Sci Rep 2015; 5: 14425. [http://dx.doi.org/10.1038/srep14425] [PMID: 26404661]

[61] Hashemian H, Jabbarvand M, Khodaparast M, Ameli K. Evaluation of corneal changes after conventional versus accelerated corneal crosslinking: a randomized controlled trial. J Refract Surg 2014; 30(12): 837-42. [http://dx.doi.org/10.3928/1081597X-20141117-02] [PMID: 25437483]

[62] Woo JH, Iyer JV, Lim L, et al. Conventional versus accelerated collagen cross-linking for keratoconus: A comparison of visual, refractive, topographic and biomechanical outcomes. Open Ophthalmol J 2017; 11: 262-72. [http://dx.doi.org/10.2174/1874364101711010262] [PMID: 29081866]

[63] Shetty R, Nagaraja H, Jayadev C, Pahuja NK, Kurian Kummelil M, Nuijts RM. Accelerated corneal collagen cross-linking in pediatric patients: two-year follow-up results. BioMed Res Int 2014; 2014: 894095. [http://dx.doi.org/10.1155/2014/894095] [PMID: 25295278]

[64] Marino GK, Torricelli AA, Giacomin N, Santhiago MR, Espindola R, Netto MV. Accelerated corneal collagen cross-linking for postoperative LASIK ectasia: Two-Year outcomes. J Refract Surg 2015; 31(6): 380-4. [http://dx.doi.org/10.3928/1081597X-20150521-04] [PMID: 26046704]

[65] Ozgurhan EB, Kara N, Cankaya KI, Kurt T, Demirok A. Accelerated corneal cross-linking in pediatric patients with keratoconus: 24-month outcomes. J Refract Surg 2014; 30(12): 843-9. [http://dx.doi.org/10.3928/1081597X-20141120-01] [PMID: 25437484]

[66] Moramarco A, Iovieno A, Sartori A, Fontana L. Corneal stromal demarcation line after accelerated crosslinking using continuous and pulsed light. J Cataract Refract Surg 2015; 41(11): 2546-51. [http://dx.doi.org/10.1016/j.jcrs.2015.04.033] [PMID: 26703505]

[67] Kanellopoulos AJ, Binder PS. Collagen Cross-Linking (CCL) with sequential topography-guided PRK: A temporizing alternative for keratoconus to penetrating keratoplasty. Cornea 2007; 26(7): 891-5. [http://dx.doi.org/10.1097/ICO.0b013e318074e424] [PMID: 17667633]

[68] Kanellopoulos AJ. Comparison of sequential vs same-day simultaneous collagen cross-linking and topography-guided PRK for treatment of keratoconus. J Refract Surg 2009; 25(9): S812-8. [http://dx.doi.org/10.3928/1081597X-20090813-10] [PMID: 19772257]

[69] Krueger RR, Kanellopoulos AJ. Stability of simultaneous topography-guided photorefractive keratectomy and riboflavin/UVA cross-linking for progressive keratoconus: Case reports. J Refract Surg 2010; 26(10): S827-32. [http://dx.doi.org/10.3928/1081597X-20100921-11] [PMID: 20954679]

[70] Stojanovic A, Zhang J, Chen X, Nitter TA, Chen S, Wang Q. Topography-guided transepithelial surface ablation followed by corneal collagen cross-linking performed in a single combined procedure for the treatment of keratoconus and pellucid marginal degeneration. J Refract Surg 2010; 26(2): 145-52. [http://dx.doi.org/10.3928/1081597X-20100121-10] [PMID: 20163079]

[71] Kymionis GD, Portaliou DM, Diakonis VF, et al. Management of post laser in situ keratomileusis ectasia with simultaneous topography guided photorefractive keratectomy and collagen cross-linking. Open Ophthalmol J 2011; 5: 11-3. [PMID: 21399769]

[72] Kanellopoulos AJ, Binder PS. Management of corneal ectasia after LASIK with combined, same-day, topography-guided partial transepithelial PRK and collagen cross-linking: the athens protocol. J Refract Surg 2011; 27(5): 323-31. [http://dx.doi.org/10.3928/1081597X-20101105-01] [PMID: 21117539]

[73] Kymionis GD, Portaliou DM, Kounis GA, Limnopoulou AN, Kontadakis GA, Grentzelos MA. Simultaneous topography-guided photorefractive keratectomy followed by corneal collagen cross-linking for keratoconus. Am J Ophthalmol 2011; 152(5): 748-55. [http://dx.doi.org/10.1016/j.ajo.2011.04.033] [PMID: 21794846]

[74] Tuwairqi WS, Sinjab MM. Safety and efficacy of simultaneous corneal collagen cross-linking with topography-guided PRK in managing lowgrade keratoconus: 1-year follow-up. J Refract Surg 2012; 28(5): 341-5. [http://dx.doi.org/10.3928/1081597X-20120316-01] [PMID: 22443804]

[75] Lin DT, Holland S, Tan JC, Moloney G. Clinical results of topography-based customized ablations in highly aberrated eyes and keratoconus/ectasia with cross-linking. J Refract Surg 2012; 28(11)(Suppl.): S841-8. [http://dx.doi.org/10.3928/1081597X-20121005-06] [PMID: 23447899] 
[76] Alessio G, L'Abbate M, Sborgia C, La Tegola MG. Photorefractive keratectomy followed by cross-linking versus cross-linking alone for management of progressive keratoconus: two-year follow-up Am J Ophthalmol. 2013;155(1):54-65 e1. [http://dx.doi.org/10.1016/j.ajo.2012.07.004]

[77] Kanellopoulos AJ, Asimellis G. Keratoconus management: Long-term stability of topography-guided normalization combined with highfluence CXL stabilization (the Athens Protocol). J Refract Surg 2014; 30(2): 88-93. [http://dx.doi.org/10.3928/1081597X-20140120-03] [PMID: 24763473]

[78] Kymionis GD, Grentzelos MA, Kounis GA, Diakonis VF, Limnopoulou AN, Panagopoulou SI. Combined transepithelial phototherapeutic keratectomy and corneal collagen cross-linking for progressive keratoconus. Ophthalmology 2012; 119(9): $1777-84$. [http://dx.doi.org/10.1016/j.ophtha.2012.03.038] [PMID: 22683058]

[79] Kymionis GD, Grentzelos MA, Kankariya VP, Pallikaris IG. Combined transepithelial phototherapeutic keratectomy and corneal collagen crosslinking for ectatic disorders: cretan protocol. J Cataract Refract Surg 2013; 39(12): 1939 [http://dx.doi.org/10.1016/j.jcrs.2013.10.003] [PMID: 24286850]

[80] Kymionis GD, Siganos CS, Tsiklis NS, et al. Long-term follow-up of Intacs in keratoconus. Am J Ophthalmol 2007; 143(2): 236-44. [http://dx.doi.org/10.1016/j.ajo.2006.10.041] [PMID: 17184717]

[81] Alió JL, Shabayek MH, Artola A. Intracorneal ring segments for keratoconus correction: Long-term follow-up. J Cataract Refract Surg 2006; 32(6): $978-85$ [http://dx.doi.org/10.1016/j.jcrs.2006.02.044] [PMID: 16814056]

[82] Kymionis GD, Tsiklis NS, Pallikaris AI, et al. Long-term follow-up of Intacs for post-LASIK corneal ectasia. Ophthalmology 2006; 113(11): 1909-17.

[http://dx.doi.org/10.1016/j.ophtha.2006.05.043] [PMID: 17074560]

[83] Chan CC, Sharma M, Wachler BS. Effect of inferior-segment Intacs with and without C3-R on keratoconus. J Cataract Refract Surg 2007; 33(1): 75-80. [http://dx.doi.org/10.1016/j.jcrs.2006.09.012] [PMID: 17189797]

[84] Coskunseven E, Jankov MR II, Hafezi F, Atun S, Arslan E, Kymionis GD. Effect of treatment sequence in combined intrastromal corneal rings and corneal collagen crosslinking for keratoconus. J Cataract Refract Surg 2009; 35(12): 2084-91. [http://dx.doi.org/10.1016/j.jcrs.2009.07.008] [PMID: 19969212]

[85] El Awady H, Shawky M, Ghanem AA. Evaluation of collagen crosslinking in keratoconus eyes with Kera intracorneal ring implantation. Eur J Ophthalmol 2012; 22(Suppl. 7): S62-8.

[http://dx.doi.org/10.5301/ejo.5000020] [PMID: 21786268]

[86] Kılıç A, Kamburoglu G, Akıncı A. Riboflavin injection into the corneal channel for combined collagen crosslinking and intrastromal corneal ring segment implantation. J Cataract Refract Surg 2012; 38(5): 878-83. [http://dx.doi.org/10.1016/j.jcrs.2011.11.041] [PMID: 22425362]

[87] Ertan A, Karacal H, Kamburoğlu G. Refractive and topographic results of transepithelial cross-linking treatment in eyes with intacs. Cornea 2009; 28(7): 719-23. [http://dx.doi.org/10.1097/ICO.0b013e318191b83d] [PMID: 19574920]

[88] Lam K, Rootman DB, Lichtinger A, Rootman DS. Post-LASIK ectasia treated with intrastromal corneal ring segments and corneal crosslinking. Digit J Ophthalmol 2013; 19(1): 1-5. [PMID: 23794955]

[89] Kymionis GD, Grentzelos MA, Karavitaki AE, Zotta P, Yoo SH, Pallikaris IG. Combined corneal collagen cross-linking and posterior chamber toric implantable collamer lens implantation for keratoconus Ophthalmic Surg Lasers Imaging. 2011;42 Online:e22-5. [http://dx.doi.org/10.3928/15428877-20110210-05]

[90] Kurian M, Nagappa S, Bhagali R, Shetty R, Shetty BK. Visual quality after posterior chamber phakic intraocular lens implantation in keratoconus. J Cataract Refract Surg 2012; 38(6): 1050-7. [http://dx.doi.org/10.1016/j.jcrs.2011.12.035] [PMID: 22624905]

[91] Fadlallah A, Dirani A, El Rami H, Cherfane G, Jarade E. Safety and visual outcome of Visian toric ICL implantation after corneal collagen cross-linking in keratoconus. J Refract Surg 2013; 29(2): 84-9. [http://dx.doi.org/10.3928/1081597X-20130117-01] [PMID: 23380407]

[92] Güell JL, Morral M, Malecaze F, Gris O, Elies D, Manero F. Collagen crosslinking and toric iris-claw phakic intraocular lens for myopic astigmatism in progressive mild to moderate keratoconus. J Cataract Refract Surg 2012; 38(3): 475-84. [http://dx.doi.org/10.1016/j.jcrs.2011.10.031] [PMID: 22261324]

[93] Kanellopoulos AJ, Dupps WJ, Seven I, Asimellis G. Toric topographically customized transepithelial, pulsed, very high-fluence, higher energy and higher riboflavin concentration collagen cross-linking in keratoconus. Case Rep Ophthalmol 2014; 5(2): 172-80. [http://dx.doi.org/10.1159/000363371] [PMID: 25076897]

[94] Sinha Roy A, Dupps WJ Jr. Patient-specific computational modeling of keratoconus progression and differential responses to collagen crosslinking. Invest Ophthalmol Vis Sci 2011; 52(12): 9174-87. [http://dx.doi.org/10.1167/iovs.11-7395] [PMID: 22039252]

[95] Nordström M, Schiller M, Fredriksson A, Behndig A. Refractive improvements and safety with topography-guided corneal crosslinking for 
keratoconus: 1-year results. Br J Ophthalmol 2017; 101(7): 920-5. [http://dx.doi.org/10.1136/bjophthalmol-2016-309210] [PMID: 27899371]

[96] Seiler TG, Fischinger I, Koller T, Zapp D, Frueh BE, Seiler T. Customized corneal cross-linking: One-Year results. Am J Ophthalmol 2016; 166: 14-21. [http://dx.doi.org/10.1016/j.ajo.2016.02.029] [PMID: 26944278]

[97] Cassagne M, Pierné K, Galiacy SD, Asfaux-Marfaing MP, Fournié P, Malecaze F. Customized topography-guided corneal collagen crosslinking for keratoconus. J Refract Surg 2017; 33(5): 290-7. [http://dx.doi.org/10.3928/1081597X-20170201-02] [PMID: 28486719]

[98] Kanellopoulos AJ. Novel myopic refractive correction with transepithelial very high-fluence collagen cross-linking applied in a customized pattern: early clinical results of a feasibility study. Clin Ophthalmol 2014; 8: 697-702. [http://dx.doi.org/10.2147/OPTH.S59934] [PMID: 24741289]

[99] Lim WK, Soh ZD, Choi HKY, Theng JTS. Epithelium-on photorefractive intrastromal cross-linking (PiXL) for reduction of low myopia. Clin Ophthalmol 2017; 11: 1205-11. [http://dx.doi.org/10.2147/OPTH.S137712] [PMID: 28721004]

[100] Kanellopoulos AJ, Asimellis G. Hyperopic correction: Clinical validation with epithelium-on and epithelium-off protocols, using variable fluence and topographically customized collagen corneal crosslinking. Clin Ophthalmol 2014; 8: 2425-33. [http://dx.doi.org/10.2147/OPTH.S68222] [PMID: 25506204]

[101] Chen YI, Chien KL, Wang IJ, et al. An interval-censored model for predicting myopic regression after laser in situ keratomileusis. Invest Ophthalmol Vis Sci 2007; 48(8): 3516-23. [http://dx.doi.org/10.1167/iovs.06-1044] [PMID: 17652718]

[102] Chayet AS, Assil KK, Montes M, Espinosa-Lagana M, Castellanos A, Tsioulias G. Regression and its mechanisms after laser in situ keratomileusis in moderate and high myopia. Ophthalmology 1998; 105(7): 1194-9. [http://dx.doi.org/10.1016/S0161-6420(98)97020-8] [PMID: 9663221]

[103] Kanellopoulos AJ, Asimellis G, Karabatsas C. Comparison of prophylactic higher fluence corneal cross-linking to control, in myopic LASIK, one year results. Clin Ophthalmol 2014; 8: 2373-81. [http://dx.doi.org/10.2147/OPTH.S68372] [PMID: 25473264]

[104] Celik HU, Alagöz N, Yildirim Y, et al. Accelerated corneal crosslinking concurrent with laser in situ keratomileusis. J Cataract Refract Surg 2012; 38(8): 1424-31. [http://dx.doi.org/10.1016/j.jcrs.2012.03.034] [PMID: 22814049]

[105] Kanellopoulos AJ, Asimellis G. Combined laser in situ keratomileusis and prophylactic high-fluence corneal collagen crosslinking for high myopia: two-year safety and efficacy. J Cataract Refract Surg 2015; 41(7): 1426-33. [http://dx.doi.org/10.1016/j.jcrs.2014.10.045] [PMID: 26287881]

[106] Kanellopoulos AJ, Kahn J. Topography-guided hyperopic LASIK with and without high irradiance collagen cross-linking: initial comparative clinical findings in a contralateral eye study of 34 consecutive patients. J Refract Surg 2012; 28(11)(Suppl.): S837-40. [http://dx.doi.org/10.3928/1081597X-20121005-05] [PMID: 23447898]

[107] Kanellopoulos AJ, Asimellis G. Epithelial remodeling after femtosecond laser-assisted high myopic LASIK: comparison of stand-alone with LASIK combined with prophylactic high-fluence cross-linking. Cornea 2014; 33(5): 463-9. [http://dx.doi.org/10.1097/ICO.0000000000000087] [PMID: 24622298]

[108] Tomita M, Yoshida Y, Yamamoto Y, Mita M, Waring G IV. In vivo confocal laser microscopy of morphologic changes after simultaneous LASIK and accelerated collagen crosslinking for myopia: one-year results. J Cataract Refract Surg 2014; 40(6): 981-90. [http://dx.doi.org/10.1016/j.jcrs.2013.10.044] [PMID: 24857441]

[109] Mazzotta C, Balestrazzi A, Traversi C, Caragiuli S, Caporossi A. In vivo confocal microscopy report after lasik with sequential accelerated corneal collagen cross-linking treatment. Case Rep Ophthalmol 2014; 5(1): 125-31. [http://dx.doi.org/10.1159/000362327] [PMID: 24847258]

[110] Tomita M. Combined laser in-situ keratomileusis and accelerated corneal cross-linking: an update. Curr Opin Ophthalmol 2016; 27(4): 304-10. [http://dx.doi.org/10.1097/ICU.0000000000000281] [PMID: 27152484]

[111] Spoerl E, Wollensak G, Seiler T. Increased resistance of crosslinked cornea against enzymatic digestion. Curr Eye Res 2004; 29(1): 35-40. [http://dx.doi.org/10.1080/02713680490513182] [PMID: 15370365]

[112] Schilde T, Kohlhaas M, Spoerl E, Pillunat LE. Enzymatic evidence of the depth dependence of stiffening on riboflavin/UVA treated corneas. Ophthalmologe 2008; 105(2): 165-9. [http://dx.doi.org/10.1007/s00347-007-1587-9] [PMID: 17955247]

[113] Arboleda A, Miller D, Cabot F, Taneja M, Aguilar MC, Alawa K, et al. Assessment of rose bengal versus riboflavin photodynamic therapy for inhibition of fungal keratitis isolates Am J Ophthalmol. 2014;158(1):64-70 e2 [http://dx.doi.org/10.1016/j.ajo.2014.04.007]

[114] Iseli HP, Thiel MA, Hafezi F, Kampmeier J, Seiler T. Ultraviolet A/riboflavin corneal cross-linking for infectious keratitis associated with corneal melts. Cornea 2008; 27(5): 590-4 [http://dx.doi.org/10.1097/ICO.0b013e318169d698] [PMID: 18520510] 
[115] Micelli Ferrari T, Leozappa M, Lorusso M, Epifani E, Micelli Ferrari L. Escherichia coli keratitis treated with ultraviolet A/riboflavin corneal cross-linking: A case report. Eur J Ophthalmol 2009; 19(2): 295-7. [http://dx.doi.org/10.1177/112067210901900221] [PMID: 19253251]

[116] Makdoumi K, Mortensen J, Crafoord S. Infectious keratitis treated with corneal crosslinking. Cornea 2010; 29(12): 1353-8. [http://dx.doi.org/10.1097/ICO.0b013e3181d2de91] [PMID: 21102196]

[117] Morén H, Malmsjö M, Mortensen J, Ohrström A. Riboflavin and ultraviolet a collagen crosslinking of the cornea for the treatment of keratitis. Cornea 2010; 29(1): 102-4. [http://dx.doi.org/10.1097/ICO.0b013e31819c4e43] [PMID: 19730094]

[118] Khan YA, Kashiwabuchi RT, Martins SA, et al. Riboflavin and ultraviolet light a therapy as an adjuvant treatment for medically refractive Acanthamoeba keratitis: report of 3 cases. Ophthalmology 2011; 118(2): 324-31. [http://dx.doi.org/10.1016/j.ophtha.2010.06.041] [PMID: 20884060]

[119] Anwar HM, El-Danasoury AM, Hashem AN. Corneal collagen crosslinking in the treatment of infectious keratitis. Clin Ophthalmol 2011; 5: 1277-80. [http://dx.doi.org/10.2147/OPTH.S24532] [PMID: 21966201]

[120] Makdoumi K, Mortensen J, Sorkhabi O, Malmvall BE, Crafoord S. UVA-riboflavin photochemical therapy of bacterial keratitis: A pilot study. Graefes Arch Clin Exp Ophthalmol 2012; 250(1): 95-102. [http://dx.doi.org/10.1007/s00417-011-1754-1] [PMID: 21874347]

[121] Kymionis GD, Kankariya VP, Kontadakis GA. Combined treatment with flap amputation, phototherapeutic keratectomy, and collagen crosslinking in severe intractable post-LASIK atypical mycobacterial infection with corneal melt. J Cataract Refract Surg 2012; $38(4)$ : 713-5. [http://dx.doi.org/10.1016/j.jcrs.2012.01.009] [PMID: 22365579]

[122] Li Z, Jhanji V, Tao X, Yu H, Chen W, Mu G. Riboflavin/ultravoilet light-mediated crosslinking for fungal keratitis. Br J Ophthalmol 2013; 97(5): 669-71. [http://dx.doi.org/10.1136/bjophthalmol-2012-302518] [PMID: 23355529]

[123] Sağlk A, Uçakhan OO, Kanpolat A. Ultraviolet A and riboflavin therapy as an adjunct in corneal ulcer refractory to medical treatment. Eye Contact Lens 2013; 39(6): 413-5. [http://dx.doi.org/10.1097/ICL.0b013e3182960fdf] [PMID: 24172067]

[124] Tabibian D, Richoz O, Riat A, Schrenzel J, Hafezi F. Accelerated photoactivated chromophore for keratitis-corneal collagen cross-linking as a first-line and sole treatment in early fungal keratitis. J Refract Surg 2014; 30(12): 855-7. [http://dx.doi.org/10.3928/1081597X-20141113-06] [PMID: 25437486]

[125] Price MO, Tenkman LR, Schrier A, Fairchild KM, Trokel SL, Price FW Jr. Photoactivated riboflavin treatment of infectious keratitis using collagen cross-linking technology. J Refract Surg 2012; 28(10): 706-13. [http://dx.doi.org/10.3928/1081597X-20120921-06] [PMID: 23062001]

[126] Richoz O, Gatzioufas Z, Hafezi F. Corneal collagen cross-linking for the treatment of acanthamoeba keratitis. Cornea 2013; 32 (10): e189. [http://dx.doi.org/10.1097/ICO.0b013e31829a689e] [PMID: 23974880]

[127] Shetty R, Nagaraja H, Jayadev C, Shivanna Y, Kugar T. Collagen crosslinking in the management of advanced non-resolving microbial keratitis. Br J Ophthalmol 2014; 98(8): 1033-5. [http://dx.doi.org/10.1136/bjophthalmol-2014-304944] [PMID: 24711659]

[128] Said DG, Elalfy MS, Gatzioufas Z, et al. Collagen cross-linking with photoactivated riboflavin (PACK-CXL) for the treatment of advanced infectious keratitis with corneal melting. Ophthalmology 2014; 121(7): 1377-82. [http://dx.doi.org/10.1016/j.ophtha.2014.01.011] [PMID: 24576886]

[129] Vajpayee RB, Shafi SN, Maharana PK, Sharma N, Jhanji V. Evaluation of corneal collagen cross-linking as an additional therapy in mycotic keratitis. Clin Experiment Ophthalmol 2015; 43(2): 103-7. [http://dx.doi.org/10.1111/ceo.12399] [PMID: 25070527]

[130] Uddaraju M, Mascarenhas J, Das MR, Radhakrishnan N, Keenan JD, Prajna L, et al. Corneal Cross-linking as an adjuvant therapy in the management of recalcitrant deep stromal fungal keratitis: A randomized trial Am J Ophthalmol. 2015;160(1):131-4 e5. [http://dx.doi.org/10.1016/j.ajo.2015.03.024]

[131] Arance-Gil Á, Gutiérrez-Ortega AR, Villa-Collar C, Nieto-Bona A, Lopes-Ferreira D, González-Méijome JM. Corneal cross-linking for Acanthamoeba keratitis in an orthokeratology patient after swimming in contaminated water. Cont Lens Anterior Eye 2014; 37(3): $224-7$. [http://dx.doi.org/10.1016/j.clae.2013.11.008] [PMID: 24355444]

[132] Bamdad S, Malekhosseini H, Khosravi A. Ultraviolet A/riboflavin collagen cross-linking for treatment of moderate bacterial corneal ulcers. Cornea 2015; 34(4): 402-6. [http://dx.doi.org/10.1097/ICO.0000000000000375] [PMID: 25710507]

[133] Spadea L. Corneal collagen cross-linking with riboflavin and UVA irradiation in pellucid marginal degeneration. J Refract Surg 2010; 26(5): 375-7. [http://dx.doi.org/10.3928/1081597X-20100114-03] [PMID: 20128533]

[134] Hassan Z, Nemeth G, Modis L, Szalai E, Berta A. Collagen cross-linking in the treatment of pellucid marginal degeneration. Indian J Ophthalmol 2014; 62(3): 367-70. 
[http://dx.doi.org/10.4103/0301-4738.109523] [PMID: 23571261]

[135] Bayraktar S, Cebeci Z, Oray M, Alparslan N. Corneal collagen cross-linking in pellucid marginal degeneration: 2 Patients, 4 eyes. Case Rep Ophthalmol Med 2015; 2015: 840687.

[http://dx.doi.org/10.1155/2015/840687] [PMID: 26078898]

[136] Kymionis GD, Karavitaki AE, Kounis GA, Portaliou DM, Yoo SH, Pallikaris IG. Management of pellucid marginal corneal degeneration with simultaneous customized photorefractive keratectomy and collagen crosslinking. J Cataract Refract Surg 2009; 35(7): 1298-301. [http://dx.doi.org/10.1016/j.jcrs.2009.03.025] [PMID: 19545822]

[137] Sharma N, Roy S, Maharana PK, et al. Outcomes of corneal collagen crosslinking in pseudophakic bullous keratopathy. Cornea 2014; 33(3): 243-6. [http://dx.doi.org/10.1097/ICO.0000000000000004] [PMID: 24452209]

[138] Kozobolis V, Labiris G, Gkika M, et al. UV-A collagen cross-linking treatment of bullous keratopathy combined with corneal ulcer. Cornea 2010; 29(2): 235-8.

[http://dx.doi.org/10.1097/ICO.0b013e3181a81802] [PMID: 20023590]

[139] Mukherjee A, Hayes S, Aslanides I, Lanchares E, Meek KM. Donor cross-linking for keratoplasty: a laboratory evaluation. Graefes Arch Clin Exp Ophthalmol 2015; 253(12): 2223-8. [http://dx.doi.org/10.1007/s00417-015-3160-6] [PMID: 26345527]

[140] Huang T, Ye R, Ouyang C, Hou C, Hu Y, Wu Q. Use of donors predisposed by corneal collagen cross-linking in penetrating keratoplasty for treating patients with keratoconus. Am J Ophthalmol 2017; 184: 115-20. [http://dx.doi.org/10.1016/j.ajo.2017.10.003] [PMID: 29032110]

[141] Robert MC, Arafat SN, Ciolino JB. Collagen cross-linking of the Boston keratoprosthesis donor carrier to prevent corneal melting in high-risk patients. Eye Contact Lens 2014; 40(6): 376-81.

[http://dx.doi.org/10.1097/ICL.0000000000000081] [PMID: 25320957]

[142] Randleman JKR. Corneal collagen cross-linking complications and their management Corneal Collagen Cross Linking. Thorofare, NJ: SLACK 2013; pp. 89-96.

[143] Lim LS, Beuerman R, Lim L, Tan DT. Late-onset deep stromal scarring after riboflavin-UV-A corneal collagen cross-linking for mild keratoconus. Arch Ophthalmol 2011; 129(3): 360-2.

[http://dx.doi.org/10.1001/archophthalmol.2011.23] [PMID: 21402996]

[144] Raiskup F, Hoyer A, Spoerl E. Permanent corneal haze after riboflavin-UVA-induced cross-linking in keratoconus. J Refract Surg 2009; 25(9): S824-8. [http://dx.doi.org/10.3928/1081597X-20090813-12] [PMID: 19772259]

[145] Kymionis GD, Portaliou DM, Diakonis VF, et al. Posterior linear stromal haze formation after simultaneous photorefractive keratectomy followed by corneal collagen cross-linking. Invest Ophthalmol Vis Sci 2010; 51(10): 5030-3.

[http://dx.doi.org/10.1167/iovs.09-5105] [PMID: 20463316]

[146] Shetty R, Kaweri L, Nuijts RM, Nagaraja H, Arora V, Kumar RS. Profile of microbial keratitis after corneal collagen cross-linking. BioMed Res Int 2014; 2014: 340509. [http://dx.doi.org/10.1155/2014/340509] [PMID: 25302296]

[147] Koller T, Mrochen M, Seiler T. Complication and failure rates after corneal crosslinking. J Cataract Refract Surg 2009; 35(8): $1358-62$. [http://dx.doi.org/10.1016/j.jcrs.2009.03.035] [PMID: 19631120]

[148] Koppen C, Vryghem JC, Gobin L, Tassignon MJ. Keratitis and corneal scarring after UVA/riboflavin cross-linking for keratoconus. J Refract Surg 2009; 25(9): S819-23. [http://dx.doi.org/10.3928/1081597X-20090813-11] [PMID: 19772258]

[149] Eberwein P, Auw-Hädrich C, Birnbaum F, Maier PC, Reinhard T. Corneal melting after cross-linking and deep lamellar keratoplasty in a keratoconus patient. Klin Monatsbl Augenheilkd 2008; 225(1): 96-8. [http://dx.doi.org/10.1055/s-2008-1027128] [PMID: 18236379]

[150] Theuring A, Spoerl E, Pillunat LE, Raiskup F. Corneal collagen cross-linking with riboflavin and ultraviolet-A light in progressive keratoconus. Results after 10-year follow-up. Ophthalmologe 2015; 112(2): 140-7. [http://dx.doi.org/10.1007/s00347-014-3114-0] [PMID: 25134461]

[151] Sharma A, Nottage JM, Mirchia K, Sharma R, Mohan K, Nirankari VS. Persistent corneal edema after collagen cross-linking for keratoconus Am J Ophthalmol. 2012;154(6):922-6 e1. [http://dx.doi.org/10.1016/j.ajo.2012.06.005]

[152] Vimalin J, Gupta N, Jambulingam M, Padmanabhan P, Madhavan HN. The effect of riboflavin-UV-A treatment on corneal limbal epithelial cells--a study on human cadaver eyes. Cornea 2012; 31(9): 1052-9. [http://dx.doi.org/10.1097/ICO.0b013e3182498902] [PMID: 22673851]

(C) 2018 Limet al

This is an open access article distributed under the terms of the Creative Commons Attribution 4.0 International Public License (CC-BY 4.0), a copy of which is available at: (https://creativecommons.org/licenses/by/4.0/legalcode). This license permits unrestricted use, distribution, and reproduction in any medium, provided the original author and source are credited. 